\title{
ON A CERTAIN LOCAL IDENTITY FOR LAPID-MAO'S CONJECTURE AND FORMAL DEGREE CONJECTURE : EVEN UNITARY GROUP CASE
}

\author{
KAZUKI MORIMOTO
}

\begin{abstract}
Lapid and Mao formulated a conjecture on an explicit formula of Whittaker Fourier coefficients of automorphic forms on quasi-split classical groups and metaplectic groups as an analogue of Ichino-Ikeda conjecture. They also showed that this conjecture is reduced to a certain local identity in the case of unitary groups. In this paper, we study even unitary group case. Indeed, we prove this local identity over $p$-adic fields. Further, we prove an equivalence between this local identity and a refined formal degree conjecture over any local field of characteristic zero. As a consequence, we prove a refined formal degree conjecture over $p$-adic fields and we get an explicit formula of Whittaker Fourier coefficients under certain assumptions.
\end{abstract}

\section{INTRODUCTION}

A relationship between periods of automorphic forms and special values of certain $L$-functions has been studied in several situations. For example, Gross and Prasad [15] conjectured a relationship between non-vanishing of Bessel period on special orthogonal groups and non-vanishing of central values of $L$-functions. Moreover, Ichino and Ikeda [19] refined the Gross-Prasad conjecture and conjectured an explicit formula between Bessel periods and central values. As an analogue of Ichino-Ikeda conjecture, Lapid and Mao 33 formulated a similar conjecture on an explicit formula of Whittaker periods for quasi-split reductive groups and metaplectic groups, which in the spirit of Sakellaridis and Venkatesh [47].

In this paper, we consider Lapid-Mao conjecture in the case of quasi-split skew-hermitian even unitary group $\mathrm{U}_{2 n}$ associated to a quartic extension $E$ of $F$. Let us recall their conjecture in our case. For an automorphic form $\varphi$ on $\mathrm{U}_{2 n}(\mathbb{A})$, we consider the Whittaker coefficient

$$
\mathcal{W}^{\psi_{N}}(\varphi):=(\operatorname{vol}(N(F) \backslash N(\mathbb{A})))^{-1} \int_{N(F) \backslash N(\mathbb{A})} \varphi(n) \psi_{N}(n)^{-1} d n .
$$

Here, $N$ is a maximal unipotent subgroup of $\mathrm{U}_{2 n}$ and $\psi_{N}$ is a non-degenerate character of $N(\mathbb{A})$. We also consider the $\mathrm{U}_{2 n}(\mathbb{A})$-invariant pairing

$$
\left(\varphi_{1}, \varphi_{2}\right):=\left(\operatorname{vol}\left(\mathrm{U}_{2 n}(F) \backslash \mathrm{U}_{2 n}(\mathbb{A})\right)\right)^{-1} \int_{\mathrm{U}_{2 n}(F) \backslash \mathrm{U}_{2 n}(\mathbb{A})} \varphi_{1}(g) \varphi_{2}(g) d g
$$

of two square-integrable automorphic forms $\varphi_{1}, \varphi_{2}$ on $\mathrm{U}_{2 n}\left(\mathbb{A}_{F}\right)$. Then we would like to construct Whittaker functional using pairing $(-,-)$. Given a finite set of places $S$ of $F$, Lapid and Mao [33] defined a stable integral

$$
\int_{N\left(\mathbb{A}^{S}\right)}^{s t} f(n) d n
$$

for a suitable class of smooth function $f$ on $N\left(\mathbb{A}^{S}\right)$ with $\mathbb{A}^{S}=\prod_{v \in S} F_{v}$. In particular, when $S$ consists of finite places, there is a sufficiently large compact open subgroup $N_{0}$ of $N\left(\mathbb{A}^{S}\right)$ such that the above integral is equal to

$$
\int_{N_{0}^{\prime}} f(n) d n
$$

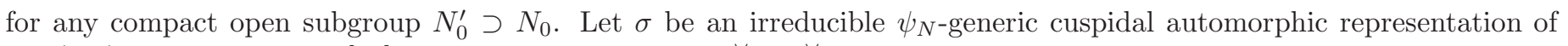
$\mathrm{U}_{2 n}\left(\mathbb{A}_{F}\right)$. Lapid and Mao [33] proved that for a $\varphi \in \sigma, \varphi^{\vee} \in \sigma^{\vee}$ and for a sufficiently large finite set $S$ of places,

$$
I\left(\varphi, \varphi^{\vee}\right):=\int_{N\left(\mathbb{A}^{S}\right)}^{s t}\left(\sigma(n) \varphi, \varphi^{\vee}\right) \psi_{N}^{-1}(n) d n
$$

is defined, and $I\left(\varphi, \varphi^{\vee}\right)$ satisfies $I\left(\sigma(n) \varphi, \sigma^{\vee}\left(n^{\prime-1}\right) \varphi^{\vee}\right)=\psi_{N}\left(n n^{\prime}\right) I\left(\varphi, \varphi^{\prime}\right)$. Then they formulated a conjecture on an explicit formula between these two Whittaker functional in the sprit of Ichino-Ikeda conjecture.

Let $\pi$ be the base change lift of $\sigma$ to $\mathrm{GL}_{2 n}\left(\mathbb{A}_{E}\right)$ constructed by Kim and Krishnamurthy [25]. It is an isobaric sum $\pi_{1} \boxplus \cdots \boxplus \pi_{k}$ where $\pi_{i}$ is an irreducible cuspidal automorphic representation of $\mathrm{GL}_{n_{i}}\left(\mathbb{A}_{E}\right)$ with $n_{1}+\cdots n_{k}=2 n$, such that $L^{S}\left(s, \pi_{i}, \mathrm{As}^{-}\right)$has a simple pole at $s=1$. Here, $L^{S}\left(s, \pi_{i}, \mathrm{As}^{-}\right)$is the partial Asai $L$-function of $\pi_{i}$ defined in [14]. Also, we have another Asai $L$-function $L^{S}\left(s, \pi_{i}, \mathrm{As}^{+}\right)$of $\pi_{i}$, which satisfies $L^{S}\left(s, \pi_{i}, \mathrm{As}^{-}\right)=L^{S}\left(s, \pi_{i} \otimes \Upsilon, \mathrm{As}^{+}\right)$where $\Upsilon$ is a character of $\mathbb{A}_{E}^{\times} / E^{\times}$whose restriction to $\mathbb{A}^{\times}$is the quadratic character $\eta_{E / F}$ corresponding to the quadratic extension $E / F$. 
Conjecture 1 (Conjecture 1.2, 5.1 in [33]). Let $\sigma$ and $\pi$ be as above. Then for any $\varphi \in \sigma$ and $\varphi^{\vee} \in \sigma^{\vee}$ and for any sufficiently large $S$ finite set of places, we have

$$
\mathcal{W}^{\psi_{N}}(\varphi) \mathcal{W}^{\psi_{N}^{-1}}\left(\varphi^{\vee}\right)=2^{1-k} \frac{\prod_{j=1}^{2 n} L^{S}\left(j, \eta_{E / F}^{j}\right)}{L^{S}\left(1, \pi, \mathrm{As}^{+}\right)}\left(\operatorname{vol}\left(N\left(\mathcal{O}_{S}\right) \backslash N\left(\mathbb{A}^{S}\right)\right)\right)^{-1} \int_{N\left(F_{S}\right)}^{s t}\left(\sigma(u) \varphi, \varphi^{\prime}\right) \psi_{N}(u)^{-1} d u .
$$

Here, $\mathcal{O}_{S}$ is the ring of $S$-integers of $F$.

In [29], for any place $v$ of $F$, Lapid and Mao defined the constant $c_{\pi_{v}}$ depending on $\pi_{v}$ (also on $\sigma_{v}$ ), which is given as a proportionality constant of two local Whittaker periods. Then they proved the following theorem.

Theorem 1.1 (Theorem 5.5 in Lapid-Mao [29]). Keep the above notation. Then for any $\varphi \in \sigma$ and $\varphi^{\vee} \in \sigma^{\vee}$ and for any sufficiently large $S$ finite set of places, we have

$$
\mathcal{W}^{\psi_{N}}(\varphi) \mathcal{W}^{\psi_{N}^{-1}}\left(\varphi^{\vee}\right)=2^{1-k}\left(\prod_{v} c_{\pi_{v}}^{-1}\right) \frac{\prod_{j=1}^{2 n} L^{S}\left(j, \eta_{E / F}^{j}\right)}{L^{S}\left(1, \pi, \operatorname{As}^{+}\right)}\left(\operatorname{vol}\left(N\left(\mathcal{O}_{S}\right) \backslash N\left(\mathbb{A}^{S}\right)\right)\right)^{-1} \int_{N\left(F_{S}\right)}^{s t}\left(\sigma(u) \varphi, \varphi^{\prime}\right) \psi_{N}(u)^{-1} d u
$$

Remark 1.1. They proved this reduction under two working assumptions; certain properties of certain local zeta integrals and the irreducibility of global descents for $\mathrm{U}_{2 n}$. The first assumption was proved in Ben-Artzi-Soudry [6] and the second assumption was proved by the author [43]. Hence, the above theorem holds without any assumption.

Because of this theorem, in order to prove Conjecture 1 it suffices to show $\prod_{v} c_{\pi_{v}}=1$. Indeed, they conjectured the following identity.

Conjecture 2 (Conjecture 5.8 in 29]). Let $v$ be a place of $F$. Then

$$
c_{\pi_{v}}=\omega_{\sigma_{v}}(-1) .
$$

In particular, this conjecture concludes that $\prod_{v} c_{\pi_{v}}=1$. We note that when $E_{v}:=E \otimes_{F} F_{v}$ is a quadratic extension, we have $\omega_{\sigma_{v}}(-1)=\omega_{\pi_{v}}(\tau)$ for $\tau \in E_{v}$ such that $\mathfrak{c}(\tau)=-\tau$ with $1 \neq \mathfrak{c} \in \operatorname{Gal}\left(E_{v} / F_{v}\right)$. Then the above conjecture is equivalent to

$$
c_{\pi_{v}}=\omega_{\pi_{v}}(\tau) .
$$

One of our local main theorems of the present paper is the proof of this conjecture in an inert case.

Theorem 1.2. Suppose that $v$ is non-split finite place (i.e. $\mathrm{U}_{2 n}\left(F_{v}\right)$ is quasi-split unitary group). Then Conjecture 2 holds.

A similar identity was proved by $[30$ in the case of metaplectic groups. In this paper, following their argument we shall prove this theorem.

This local identity is important not only for Lapid-Mao conjecture but also for formal degree conjecture in HiragaIchino-Ikeda [18. Using an observation by Gross-Reeder [16, Gan-Ichino [12, 14.5] formulated a refinement of this conjecture for classical groups. We call this refinement refined formal degree conjecture. In [20], Ichino-Lapid-Mao showed that a similar local identity to Conjecture 2 proved in 30 is equivalent to refined formal degree conjecture for metaplectic groups. As a consequence of [30, they proved refined formal degree conjecture in this case. In a similar argument as 20, we prove the equivalence between refined formal degree conjecture for $\mathrm{U}_{2 n}$ and Theorem 1.2. As a consequence, we can prove refined formal degree conjecture.

Theorem 1.3. Let $F$ be a non-arhimedean local field of characteristic zero and $E$ a quadratic extension of $F$. Let $\pi$ be an irreducible representation of $\mathrm{GL}_{2 n}(E)$ of the form $\pi=\tau_{1} \times \cdots \times \tau_{k}$ where $\tau_{i}$ are mutually inequivalent irreducible discrete series representations of $\mathrm{GL}_{n_{i}}(E)$ such that $n=n_{1}+\cdots+n_{k}$ and $L\left(s, \tau_{i}, \mathrm{As}^{+}\right)$has a pole at $s=0$. Write $\sigma=\mathcal{D}_{\psi^{-1}}^{\Upsilon^{-1}}(\mathfrak{c}(\pi))$, which is an irreducible generic discrete series representation of $G^{\prime}$ Then we have

$$
d_{\psi}=2^{k} \lambda(E / F)^{n} \omega_{\sigma}(-1) \gamma\left(1, \mathfrak{c}(\pi), \mathrm{As}^{-}, \psi\right) d_{\sigma} .
$$

Here, $d_{\sigma}$ is the formal degree (See Section 8) and $d_{\psi}$ is a certain measure on $\mathrm{U}_{2 n}(F)$ (See Section 2.4).

Remark 1.2. Recently, Beuzart-Plessis [7] proved the original formal degree conjecture by [18] for any unitary groups using a different method.

On the other hand, we can show refined formal degree conjecture for $\mathrm{U}_{2 n}$ over real field using computations in [18. In a similar argument as the non-archimedean case, we can prove the equivalence between refined formal degree conjecture and Conjecture 2 Hence, we obtain the following result.

Theorem 1.4. Suppose that $v$ is a real place and that $E_{v} \simeq \mathbb{C}$, i.e. $\mathrm{U}_{2 n}\left(F_{v}\right) \simeq \mathrm{U}_{2 n}(\mathbb{R})$. Then Conjecture 2 holds for diecerete series representations of $\mathrm{U}_{2 n}(\mathbb{R})$.

As a corollary of Theorem 1.2. Theorem [1.4 and [29, Lemma 5.4], the following global formula follows. 
Corollary 1.1. Let $\sigma$ be as in Conjecture 1. Suppose that $F$ is totally real field. Assume that for a places $v$ of $F$,

$$
\begin{cases}\sigma_{v} \text { is unramified } & \text { if } v \text { is split finite place or } v \mid 2 \\ \sigma_{v} \text { is discrete series } & \text { if } v \text { is real place }\end{cases}
$$

Then the formula in Conjecture 1 holds.

In [11, we proved the refined Gross-Prasad conjecture for special Bessel periods for $\mathrm{SO}(2 n+1)$ using the explicit formula of Whittaker periods of metaplectic groups by Lapid-Mao 30. In a similar computation as [11, Corollary 1.1 should yield a formula of Bessel periods for $\left(\mathrm{U}_{2 n}, \mathrm{U}_{1}\right)$. In our future work, we will study this problem.

This paper is organized as follows. In Section 2, we define basic notations. In Section 3, we formulate Lapid-Mao's local conjecture and reduce it to tempered case. In Section 4-6, following the idea by Lapid and Mao [30, we rewrite our required identity by another local identity using local analysis and certain functional equations. (See [30, Section 4] for the idea of the proof). Most parts are proved in a similar argument as [30. Hence, we give a proof only when there are non-trivial difference, and we only give statements if the proof is essentially same as the corresponding results in [30. In Section 7, we complete the proof of Theorem 1.2. In Section 8, we prove Theorem 1.3 and Theorem 1.4

Acknowledgements. The author express his deep gratitude to Erez Lapid for suggesting him to the problems in the present paper and for useful discussion.

\section{Notation And Preliminaries}

\subsection{Groups, homomorphisms and group elements.}

- Let $F$ be a local field of characterstic zero and $E$ a quadratic extension of $F$. Let $\mathfrak{c}$ be a non-trivial element of $\operatorname{Gal}(E / F)$. We take $\tau \in E$ such that $\mathfrak{c}(\tau)=-\tau$.

- $\eta_{E / F}$ denotes the quadratic character of $F^{\times}$corresponding to $E / F$.

- Fix a character $\Upsilon$ of $E^{\times}$such that $\left.\Upsilon\right|_{F^{\times}}=\eta_{E / F}$.

- $I_{m}$ is the identity matrix in $\mathrm{GL}_{m}, w_{m}$ is the $m \times m$-matrix with ones on the non- principal diagonal and zeros elsewhere.

- For any group $Q, Z_{Q}$ is the center of $Q ; e$ is the identity element of $Q$. We denote the modulus function of $Q$ (i.e., the quotient of a right Haar measure by a left Haar measure) by $\delta_{Q}$.

- $\mathrm{Mat}_{m}$ is the vector space of $m \times m$ matrices over $F$.

- for $x=\left(x_{i} j\right) \in \operatorname{Mat}(E), x^{\mathfrak{c}}$ denotes the matrix $\left(\mathfrak{c}\left(x_{i j}\right)\right)$

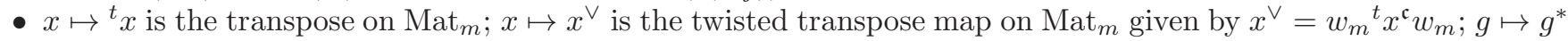
is the outer automorphism of $\mathrm{GL}_{m}$ given by $g^{*}=w_{m}^{-1}\left({ }^{t} g^{\mathfrak{c}}\right)^{-1} w_{m}$.

- $\mathfrak{u}_{n}=\left\{x \in \operatorname{Mat}_{n}(E): x^{\vee}=x\right\}$

- $\mathbb{M}=\mathrm{GL}_{2 n}(E), \mathbb{M}=\mathrm{GL}_{n}(E)$

- $G=\mathrm{U}_{4 n}=\left\{g \in \mathrm{GL}_{4 n}(E):{ }^{t} g\left(\begin{array}{cc}w_{2 n} & w_{2 n}\end{array}\right) g=\left(\begin{array}{ll} & w_{2 n} \\ -w_{2 n} & \end{array}\right)\right\}$

- $G^{\prime}=\mathrm{U}_{2 n}=\left\{g \in \mathrm{GL}_{2 n}(E):{ }^{t} g\left(\begin{array}{cc}w_{n} \\ -w_{n}\end{array}\right) g=\left(\begin{array}{cc}w_{n} \\ -w_{n}\end{array}\right)\right\}$

- $G^{\prime}$ is embedded as a subgroup of $G$ via $g \mapsto \eta(g)=\operatorname{diag}\left(I_{n}, g, I_{n}\right)$.

- $P=M U$ (resp., $P^{\prime}=M^{\prime} U^{\prime}$ ) is the Siegel parabolic subgroup of $G$ (resp., $G^{\prime}$ ), with its standard Levi decomposition.

- $\bar{P}={ }^{t} P$ is the opposite parabolic of $P$, with unipotent radical $\bar{U}={ }^{t} U$.

- We use the isomorphism $\varrho(g)=\operatorname{diag}\left(g, g^{*}\right)$ to identify $\mathbb{M}$ with $M \subset G$. Similarly for $\varrho^{\prime}: \mathbb{M}^{\prime} \rightarrow M^{\prime} \subset G^{\prime}$.

- We use the embeddings $\eta_{\mathbb{M}}(g)=\operatorname{diag}\left(g, I_{n}\right)$ and $\eta_{\mathbb{M}}^{\vee}(g)=\operatorname{diag}\left(I_{n}, g\right)$ to identify $\mathbb{M}^{\prime}$ with subgroups of $\mathbb{M}$. We also set $\eta_{M}=\varrho \circ \eta_{\mathbb{M}}$ and $\eta_{\mathbb{M}}^{\vee}=\varrho \circ \eta_{\mathbb{M}^{\prime}}=\eta \circ \varrho^{\prime}$

- $K$ is the standard maximal compact subgroup of $G$. (In the p-adic case it consists of the matrices with integral entries.)

- $N$ is the standard maximal unipotent subgroup of $G$ consisting of upper unitriangular matrices; $T$ is the maximal torus of $\mathrm{G}$ consisting of diagonal matrices; $B=T N$ is the Borel subgroup of $G$.

- For any subgroup $X$ of $G$ we write $X^{\prime}=\eta^{-1}(X), X_{M}=X \cap M$ and $X_{\mathbb{M}}=\varrho^{-1}\left(X_{M}\right)$; similarly $X_{M^{\prime}}^{\prime}=X^{\prime} \cap M^{\prime}$ and $X_{\mathbb{M}^{\prime}}^{\prime}=\left(\varrho^{\prime}\right)^{-1}\left(X_{M^{\prime}}^{\prime}\right)$.

- $\ell_{\mathbb{M}}:$ Mat $_{n} \rightarrow N_{\mathbb{M}}$ is the group embedding given by $\ell_{\mathbb{M}}(x)=\left(\begin{array}{cc}I_{n} & x \\ I_{n}\end{array}\right)$ and $\ell_{M}=\varrho \circ \ell_{\mathbb{M}}$.

- $\ell: \mathfrak{u}_{2 n} \rightarrow U$ is the group isomorphism given by $\ell(x)=\left(\begin{array}{cc}I_{n} & x \\ & I_{n}\end{array}\right)$.

- $\xi_{m}=(0, \ldots, 0,1) \in F^{m}$

- $\mathcal{P}$ is the mirabolic subgroup of $\mathbb{M}$ consisting of the elements $g$ such that $\xi_{2 n} g=\xi_{2 n}$

- Put $H_{\mathbb{M}}=\mathrm{GL}_{2 n}(F)$ 
- $\mathfrak{t}=\operatorname{diag}(1,-1, \ldots, 1,-1) \in \mathbb{M}$.

- $w_{0}^{\prime}=\left({ }_{-w_{n}}{ }^{w_{n}}\right) \in G^{\prime}$ represents the longest Weyl element of $G^{\prime}$.

- $w_{U}=\left(\begin{array}{cc}I_{2 n} \\ -I_{2 n}\end{array}\right) \in G$ represents the longest $M$-reduced Weyl element of $G^{\prime}$

- $w_{U^{\prime}}^{\prime}=\left(\begin{array}{c}I_{n} \\ I_{n}\end{array}\right) \in G^{\prime}$ represents the longest $M^{\prime}$-reduced Weyl element of $G^{\prime}$

- $w_{0}^{\mathbb{M}}=w_{2 n} \in \mathbb{M}$ represents the longest Weyl element of $\mathbb{M} ; w_{0}^{M}=\varrho\left(w_{0}^{\mathbb{M}}\right)$.

- $w_{0}^{\mathbb{M}^{\prime}}=w_{n} \in \mathbb{M}$ represents the longest Weyl element of $\mathbb{M} ; w_{0}^{M^{\prime}}=\varrho\left(w_{0}^{\mathbb{M}^{\prime}}\right)$.

- $w_{2 n, n}=\left({ }_{I_{n}} I_{n}\right) \in \mathbb{M}, w_{2 n, n}^{\prime}=\left(\begin{array}{c}I_{n} \\ w_{0}^{\mathbb{M}^{\prime}}\end{array}\right) \in \mathbb{M}$

- $\gamma=w_{U} \eta\left(w_{U^{\prime}}^{\prime}\right)^{-1}=\left(\begin{array}{ccc}I_{n} & \\ -I_{n} & & I_{n} \\ & & I_{n}\end{array}\right) \in G$

- $\mathfrak{d}=\operatorname{diag}\left(1,-1, \ldots,(-1)^{n-1}\right) \in \operatorname{Mat}_{n}, \varepsilon_{1}=(\hat{w} \gamma)^{-1} \varrho\left(\varepsilon_{3}\right) w_{U}=\ell_{M}\left((-1)^{n} \mathfrak{d}\right), \varepsilon_{2}=\ell_{\mathbb{M}}(\mathfrak{d}), \varepsilon_{3}=w_{2 n, n}^{\prime} \varepsilon_{2}, \varepsilon_{4}=$ $\ell_{\mathbb{M}}\left(-\frac{1}{2} \mathfrak{d} w_{0}^{\mathbb{M}^{\prime}}\right)$.

- $V$ (resp. $V^{\#}$ ) is the unipotent radical of the standard parabolic subgroup of $G$ with $\operatorname{Levi}_{\mathrm{GL}_{1}}(E)^{n} \times \mathrm{U}_{2 n}($ resp. $\left.\mathrm{GL}_{1}(E)^{n-1} \times \mathrm{U}_{2 n+2}\right)$. Thus, $N=\eta\left(N^{\prime}\right) \ltimes V, V^{\#}$ is normal in $V$ and $V / V^{\#}$ is isomorphic to the Heisenberg group of dimension $2 n+1$ over $E$. Also $V=V_{M} \ltimes V_{U}$ where $V_{U}=V \cap U=\left\{\ell\left(\begin{array}{cc}x & y \\ x^{\vee}\end{array}\right): x \in \operatorname{Mat}_{n}(E), y \in \mathfrak{u}_{n}\right\}$

- $V_{-}=V_{M}^{\#} \ltimes V_{U}$ (Recall $V_{M}^{\#}=V^{\#} \cap M$ by our convention)

- $V_{\gamma}=V \cap \gamma^{-1} N \gamma=\eta\left(w_{U^{\prime}}^{\prime}\right) V_{M} \eta\left(w_{U^{\prime}}^{\prime}\right)=\eta_{M}\left(N_{\mathbb{M}^{\prime}}^{\prime}\right) \ltimes\left\{\ell\left({ }^{x}{ }_{x} \vee\right): x \in \operatorname{Mat}_{n}(E)\right\} \subset V_{-}$

- $V_{+} \subset V$ is the image under $\ell_{M}$ of the space of $n \times n$-matrices over $E$ whose rows are zero except possibly for the last one. Thus, $V=V_{+} \ltimes V_{-}$. For $c=\ell_{M}(x) \in V_{+}$we denote by $\underline{c}$ the last row of $x$.

- $N^{\#}=V_{-} \rtimes \eta\left(N^{\prime}\right)$.It is the stabilizer in $N$ of the character $\psi_{U}$ defined below.

- $N_{\mathbb{M}}^{b}=\left(N_{\mathbb{M}}^{\#}\right)^{*}$

- $J$ is the subspace of Mat $_{n}$ consisting of the matrices whose first column is zero.

- $\bar{R}=\left\{\ell\left(\begin{array}{cc}I_{n} & \\ x & { }^{t} n\end{array}\right): x \in J, n \in N_{\mathbb{M}^{\prime}}^{\prime}\right\}$.

2.2. Characters. We fix a non-trivial additive character $\psi_{F}$ of $F$ and define an additive character $\psi$ of $E$ by $\psi(x)=$ $\psi_{F}\left(\frac{x+\mathfrak{c}(x)}{2}\right)$ for $x \in E$.

- $\psi_{N_{\mathbb{M}}}(u)=\psi\left(u_{1,2}+\cdots+u_{2 n-1,2 n}\right)$

- $\psi_{N_{M}} \circ \varrho=\psi_{N_{\mathbb{M}}}$

- $\psi_{N_{\mathbb{M}^{\prime}}^{\prime}}(u)=\psi\left(u_{1,2}^{\prime}+\cdots+u_{n-1, n}^{\prime}\right)$

- $\psi_{N_{M^{\prime}}^{\prime}} \circ \varrho^{\prime}=\psi_{N_{\mathbb{M}^{\prime}}^{\prime}}$.

- $\psi_{N^{\prime}}(n u)=\psi_{N_{\mathbb{M}^{\prime}}^{\prime}}(n) \psi\left(\frac{1}{2} u_{n, n+1}\right)^{-1}, n \in N_{M^{\prime}}^{\prime}, u \in U^{\prime}$

- $\psi_{N}(n u)=\psi_{N_{M}}(n), n \in N_{M}, u \in U$ (a degenerate character). Then $\psi_{N_{M^{\prime}}^{\prime}}(u)=\psi_{N}\left(\gamma \eta(u) \gamma^{-1}\right)$

- $\psi_{V}(v u)=\psi_{N_{M}}\left(w_{U} u w_{U}^{-1}\right)$ where we write an element of $V$ by $v u$ so that $u$ fixes $e_{1}, \ldots e_{n}, v$ fixes $e_{n+1}, \ldots e_{n+1}, \ldots e_{-1-n}$

- $\psi_{V_{-}}(v u)=\psi_{N_{M}}(v)^{-1} \psi_{U}(u), v \in V_{M}^{\#}, u \in V_{U}$. (Note that this is not a restriction of $\psi_{V}$ to $V_{-}$.)

- $\psi_{U}(\ell(v))=\psi\left(\frac{1}{2}\left(v_{n, n+1}-v_{2 n, 1}\right)\right)$

- $\psi_{\bar{U}}(\bar{v})=\psi\left(v_{2 n+1,1}\right), \bar{v} \in \bar{U}$.

- $\Upsilon_{M}(\varrho(g))=\Upsilon(\operatorname{det} g), g \in \mathbb{M}$ and $\Upsilon_{M^{\prime}}\left(\varrho^{\prime}\left(g^{\prime}\right)\right)=\Upsilon\left(\operatorname{det} g^{\prime}\right), g^{\prime} \in \mathbb{M}^{\prime}$

\subsection{Other notation.}

- We use the notation $a \ll_{d} b$ to mean that $a \leq c b$ with $c>0$ a constant depending on $d$.

- For any $g \in G$ define $\nu(g) \in \mathbb{R}>0$ by $\nu(u \varrho(m) k)=\|\operatorname{det} m\|_{E}$ for any $u \in U, m \in \mathbb{M}, k \in K$. Let $\nu^{\prime}(g)=\nu(\eta(g))$ for $g \in G^{\prime}$.

- $\mathcal{C} \mathcal{S G R}(Q)$ is the set of compact open subgroups of a topological group $Q$.

- For an $\ell$-group $Q$ let $C(Q)$ (resp., $\mathcal{S}(Q)$ ) be the space of continuous (resp., Schwartz) functions on $Q$ respectively.

- When $F$ is $p$-adic, if $Q^{\prime}$ is a closed subgroup of $Q$ and $\chi$ is a character of $Q^{\prime}$, we denote by $C\left(Q^{\prime} \backslash Q, \chi\right)($ resp., $\left.\left.C^{\text {sm }}\left(Q^{\prime} \backslash Q, \chi\right), C_{c}^{\infty}\left(Q^{\prime} \backslash Q, \chi\right)\right)\right)$ the spaces of continuous (resp. $Q$-smooth, smooth and compactly supported modulo $\left.Q^{\prime}\right)$ complex-valued left $\left(Q^{\prime}, \chi\right)$-equivariant functions on $Q$.

- For an $\ell$-group $Q$ we write $\operatorname{Irr} Q$ for the set of equivalence classes of irreducible representations of $Q$. If $Q$ is reductive we also write $\operatorname{Irr}_{\text {sqr }} Q$ and $\operatorname{Irr}_{\text {temp }} Q$ for the subsets of irreducible unitary square-integrable (modulo center) and tempered representations respectively. We write $\operatorname{Irr}_{\text {gen }} \mathbb{M}$ and $\operatorname{Irr}_{\mathrm{ut}} \mathbb{M}$ for the subset of irreducible generic representations of $\mathbb{M}$ and representations of unitary type (see below), respectively. For the set of irreducible generic representations of $G$ we use the notation $\operatorname{Irr}_{\text {gen }, \psi_{N^{\prime}}} G^{\prime}$ to emphasize the dependence on the character $\psi_{N^{\prime}}$.

- For $\pi \in \operatorname{Irr} Q$, let $\pi^{\vee}$ be the contragredient of $\pi$.

- For $\pi \in \operatorname{Irr}_{\text {gen }} \mathbb{M}, \mathbb{W}^{\psi_{N_{\mathbb{M}}}}(\pi)$ denotes the (uniquely determined) Whittaker space of $\pi$ with respect to the character

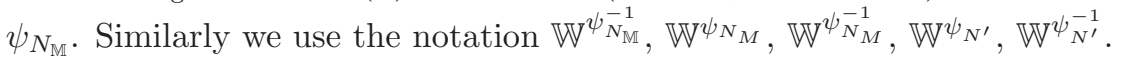


- For $\pi \in \operatorname{Irr}_{\text {gen }} \mathbb{M}$ let $\operatorname{Ind}\left(\mathbb{W}^{\psi_{N_{M}}}(\pi)\right)$ be the space of smooth left $U$-invariant functions $W: G \rightarrow \mathbb{C}$ such that for all $g \in G$, the function $m \mapsto \delta_{P}(m)^{-\frac{1}{2}} W(m g)$ on $M$ belongs to $\mathbb{W}^{\psi_{N_{M}}}(\pi)$. Similarly define $\operatorname{Ind}\left(\mathbb{W}^{\psi_{N_{M}}^{-1}}(\pi)\right)$

- If a group $G_{0}$ acts on a vector space $W$ and $H_{0}$ is a subgroup of $G_{0}$, we denote by $W^{H_{0}}$ the subspace of $H_{0}$-fixed points.

- We use the following bracket notation for iterated integrals: $\iint\left(\iint \ldots\right) \ldots$ implies that the inner integrals converge as a double integral and after evaluating them, the outer double integral is absolutely convergent.

2.4. Measures. The Lie algebra $\mathfrak{M}$ of $\operatorname{Res}_{E / F} \mathrm{GL}_{m}$ consists of the $m \times m$-matrices $X$ over $E$. Let $\mathfrak{M}_{\mathcal{O}}$ be the lattice of integral matrices in $\mathfrak{M}$. For any algebraic subgroup $\mathbf{Q}$ of $\operatorname{Res}_{E / F} \mathrm{GL}_{m}$ defined over $F$, let $\mathfrak{q} \subset \mathfrak{M}$ be the Lie algebra of $\mathbf{Q}$. The lattice $\mathfrak{q} \cap \mathfrak{M}_{\mathcal{O}}$ of $\mathfrak{q}$ gives rise to a gauge form of $\mathbf{Q}$ (determined up to multiplication by an element of $\mathcal{O}_{E}^{*}$ ) and we use it (together with $\psi$ ) to define a Haar measure on $Q$ by the recipe of Kneser [26. For example, when $n=1$, the measure on $N_{\mathbb{M}}=\left\{\left(\begin{array}{cc}1 & x \\ 1\end{array}\right): x \in E\right\} \simeq E$ is the self-dual Haar measure $d x$ on $E$ with respect to $\psi$. It is written as follows using measure on $F$. Let $d x_{i}(i=1,2)$ and $d y_{i}(i=1,2)$ be self-dual Haar measures on $F$ with respect to $\psi_{F}$, i.e. the measure on $F$ such that

$$
\int_{F} \int_{F} f\left(x_{i}\right) \psi_{F}\left(x_{i} y_{i}\right) d x_{i} d y_{i}=f(0)
$$

for a smooth function $f$ on $F$, provided the integral converges. We define a Haar measure on $E$ by

$$
d z_{i}=|\tau|^{\frac{1}{2}} d x_{i} d y_{i} \quad \text { with } \quad z_{i}=x_{i}+\tau y_{i}
$$

Then for a smooth function $g$ on $E$, we have

$$
\int_{E} \int_{E} g\left(z_{1}\right) \psi\left(z_{1} z_{2}\right) d z_{1} d z_{2}=g(0)
$$

provided the integral converges. Namely, $d z_{i}$ are self-dual Haar measures with respect to $\psi$. Further, we note that for $f \in L^{1}(F)$, we have

$$
\int_{F} \int_{F} f(x) \psi(a x y) d x d y=|a|^{-1} \cdot f(0)
$$

and

$$
\int_{F}\left(\int_{F \backslash E} f(x) \psi_{E}(\tau x y) d x\right) d y=|\tau|^{-\frac{1}{2}} f(0), \quad \int_{F}\left(\int_{F \backslash E} f(x) \psi_{E}\left(\tau^{-1} x y\right) d x\right) d y=|\tau|^{\frac{1}{2}} f(0) .
$$

2.5. Weil representation. Let $Y$ be a $2 n$-dimensional space over $E$, equipped with a non-degenerate, anti-Hermitian form $(\cdot, \cdot)$. Assume that it has a maximal isotropic subspace of dimension $n$ over $E$. We denote its isometry group by $\mathrm{U}(Y) \simeq \mathrm{U}_{2 n}$. We may view $Y$ as symplectic space over $F$ with the symplectic form $\langle\cdot, \cdot\rangle=\operatorname{Tr}_{E / L}(\cdot, \cdot)$. Then it is $4 n$ dimensional vector space over $F$, and we denote this space by $Y^{\prime}$. Let $\widetilde{\operatorname{Sp}}\left(Y^{\prime}\right)$ be the metaplectic cover of $\operatorname{Sp}\left(Y^{\prime}\right)$ with respect to the cocycle given in [39, p.59] (see also [13, p.455]). It is clear that $\mathrm{U}(Y) \subset \widetilde{\mathrm{Sp}}\left(Y^{\prime}\right)$, and we know that $\widetilde{\operatorname{Sp}}\left(Y^{\prime}\right)$ splits over $\mathrm{U}(Y)$. Fix a character $\Upsilon$ of $E^{\times}$such that $\left.\Upsilon\right|_{F^{\times}}=\eta_{E / F}$, and we choose the splitting as in [14, p.9] corresponding to $\Upsilon$. Let us write

$$
Y=Y^{+}+Y^{-}
$$

as a direct sum of two maximal isotropic subspaces of $Y$, which are duality under $(\cdot, \cdot)$. When we consider $Y^{ \pm}$as subspace of $Y^{\prime}$, they are isotropic for $\langle\cdot, \cdot\rangle$, and thus in duality under $\langle\cdot, \cdot\rangle$. Consider the Weil representation $\omega_{\psi}^{\Upsilon}$ of the group $\mathcal{H}_{Y} \rtimes \widetilde{\operatorname{Sp}}\left(Y^{\prime}\right)$ on $\Phi \in \mathcal{S}\left(E^{n}\right)$, where $\mathcal{H}_{Y}:=Y \oplus F$ is the Heisenberg group attached to $Y$ with the multiplication

$$
\left(w_{1}, t_{1}\right) \cdot\left(w_{2}, t_{2}\right)=\left(w_{1}+w_{2}, t_{1}+t_{2}+\frac{1}{2} \operatorname{Tr}_{E / L}\left(w_{1}, w_{2}\right)\right) .
$$

Explicitly, for any $\Phi \in \mathcal{S}\left(Y^{+}\right)$and $X \in Y^{+}$, the action of $\mathcal{H}_{Y}$ is given by

$$
\begin{aligned}
& \omega_{\psi}^{\Upsilon}(a, 0) \Phi(X)=\Phi(X+a), \quad a \in Y^{+}, \\
& \left.\omega_{\psi}^{\Upsilon}(b, 0) \Phi(X)=\psi(\langle X, b\rangle)\right) \Phi(X), \quad b \in Y^{-}, \\
& \omega_{\psi}^{\Upsilon}(0, t) \Phi(X)=\psi(t) \Phi(X), \quad t \in F
\end{aligned}
$$

while the action of $\widetilde{\mathrm{Sp}}\left(Y^{\prime}\right)$ is (partially) given by

$$
\begin{aligned}
& \omega_{\psi}^{\Upsilon}\left(\left(\begin{array}{ll}
g & \\
& g^{*}
\end{array}\right), \varepsilon\right) \Phi(X)=\varepsilon \gamma_{\psi}\left(N_{E / L}(\operatorname{det} g)\right) \Upsilon(\operatorname{det} g)|\operatorname{det} g|^{1 / 2} \Phi(X \cdot g) \\
& \left.\omega_{\psi}^{\Upsilon}\left(\left(\begin{array}{rr}
I & y \\
& I
\end{array}\right), \varepsilon\right) \Phi(X)=\varepsilon \psi\left(\frac{1}{2}\langle X, X \cdot y\rangle\right)\right) \Phi(X)
\end{aligned}
$$


We now take $Y=E^{2 n}$ with the skew-hermitian form

$$
\left\langle\left(x_{1}, \ldots, x_{2 n}\right),\left(y_{1}, \ldots, y_{2 n}\right)\right\rangle=\sum_{i=1}^{n} x_{i} \mathfrak{c}\left(y_{2 n+1-i}\right)-\sum_{i=1}^{n} y_{i} \mathfrak{c}\left(x_{2 n+1-i}\right)
$$

and the standard polarization $Y^{+}=\left\{\left(y_{1}, \ldots, y_{n}, 0 \ldots, 0\right)\right\}$ and $Y^{-}=\left\{\left(0 \ldots, 0, y_{1}, \ldots, y_{n}\right)\right\}$ with the standard basis $e_{1}, \ldots, e_{n}, e_{-n}, \ldots, e_{-1}$. For $\Phi \in \mathcal{S}\left(E^{n}\right)$, we define the Fourier transform

$$
\hat{\Phi}(X)=\int_{E^{n}} \Phi\left(X^{\prime}\right) \psi\left(\left\langle X, X^{\prime}\left({ }_{-I_{n}}^{I_{n}}\right)\right\rangle\right)
$$

Then, reapplied on $\mathcal{S}\left(E^{n}\right)$, the Weil representation satisfies

$$
\begin{aligned}
\omega_{\psi}^{\Upsilon}\left(\varrho^{\prime}(g)\right) \Phi(X) & =\alpha_{\psi}\left(\varrho^{\prime}(g)\right) \Upsilon_{M^{\prime}}\left(\varrho^{\prime}(g)\right) \nu^{\prime}\left(\varrho^{\prime}(g)\right)^{1 / 2} \Phi(X \cdot g) \\
\omega_{\psi}^{\Upsilon}\left(\left(\begin{array}{cc}
I & y \\
& I
\end{array}\right)\right) \Phi(X) & \left.=\psi\left(\frac{1}{2}\langle X, X \cdot y\rangle\right)\right) \Phi(X) \\
\omega_{\psi}^{\Upsilon}\left(w_{U^{\prime}}^{\prime}\right) \Phi(X) & =\alpha_{\psi}\left(w_{U^{\prime}}^{\prime}\right) \hat{\Phi}(X)
\end{aligned}
$$

where $\alpha_{\psi}(\cdot)$ is a certain eight root of unity, which satisfies $\alpha_{\psi}(\cdot)^{-1}=\alpha_{\psi^{-1}}(\cdot)$

Let $V_{0} \subset V$ be the unipotent radical of the standard parabolic subgroup of $G$ with Levi $\operatorname{GL}_{1}(E)^{n-1} \times \mathrm{U}_{2 n+2}$. The the map

$$
v \mapsto v_{\mathcal{H}}:=\left(\left(v_{n, n+j}\right)_{j=1, \ldots, 2 n}, \frac{1}{2} \operatorname{Tr}_{E / L}\left(v_{n, 3 n+1}\right)\right)
$$

gives an isomorphism from $V / V_{0}$ to a Heisenberg group $\mathcal{H}_{Y}$. Then we may regard $\omega_{\psi}^{\Upsilon}$ as a representation of $\widetilde{\operatorname{Sp}(Y)} \ltimes V / V_{0}$. Further, we extend $\omega_{\psi}^{\Upsilon}$ to a representation $\omega_{\psi_{N_{\mathbb{M}}}^{\Upsilon}}^{\Upsilon}$ of $V \rtimes G^{\prime}$ by setting

$$
\omega_{\psi}^{\Upsilon}(v g) \Phi=\psi_{V}(v) \omega_{\psi}^{\Upsilon}\left(v_{\mathcal{H}}\right)\left(\omega_{\psi}^{\Upsilon}(g) \Phi\right), \quad v \in V, g \in G^{\prime} .
$$

2.6. Stable integral. Many integral studied in this paper do not absolutely converge. Instead of such integrals, we shall use the notion of stable integrals as in 30 . For the convenience to the reader, we shall recall the notion of stable integral. Suppose that $F$ is $p$-adic. Let $U_{0}$ be a unipotent subgroup over $F$ with a fixed Haar measure on $U_{0}$.

Definition 2.1 (Definition 2.1 in [30]). Let $f$ be a smooth function on $U_{0}$. We say that $f$ has a stable integral over $U_{0}$ if there exists $U_{1} \in \mathcal{C} \mathcal{S G R}\left(U_{0}\right)$ such that for any $U_{2} \in \mathcal{C} \mathcal{S G R}\left(U_{0}\right)$ containing $U_{1}$ we have

$$
\int_{U_{2}} f(u) d u=\int_{U_{1}} f(u) d u .
$$

In this case we write $\int_{U_{0}}^{\mathrm{st}} f(u) d u$ for the common value (2.11) and say that $\int_{U_{0}}^{\mathrm{st}} f(u) d u$ stabilizes at $U_{1}$. In other words, $\int_{U_{0}}^{\text {st }} f(u) d u$ is the limit of the net $\left(\int_{U_{1}} f(u) d u\right)_{U_{1} \in \mathcal{C S G R}\left(U_{0}\right)}$ with respect to the discrete topology of $\mathbb{C}$.

We also define uniformally stable integral (see [30, pp.10])

Definition 2.2. Given a family of functions $f_{x} \in C^{\mathrm{sm}}\left(U_{0}\right)$, we say that the integral $\int_{U_{0}}^{\mathrm{st}} f_{x}(u) d u$ stabilizes uniformly in $x$ if $U_{1}$ as above can be chosen independently of $x$. Similarly, if $x$ ranges over a topological space $X$ then we say that $\int_{U_{0}}^{\text {st }} f_{x}(u) d u$ stabilizes locally uniformly in $x$ if any $y \in X$ admits a neighborhood on which $\int_{U_{0}}^{\text {st }} f_{x}(u) d u$ stabilizes uniformly.

\section{Statement of Main Result}

3.1. Local Fourier-Jacobi transform. For any $f \in C(G)$ and $s \in \mathbb{C}$, define $f_{s}(g)=f(g) \nu(g)^{s}, g \in G$. Let $\pi \in \operatorname{Irr}_{\text {gen }} \mathbb{M}$ with Whittaker model $W^{\psi_{N_{\mathbb{M}}}}(\pi)$. Let $\operatorname{Ind}\left(W^{\psi_{N_{\mathbb{M}}}}(\pi)\right)$ be the space of $G$-smooth left $U$-invariant functions $W: G \rightarrow \mathbb{C}$ such that for all $g \in G$, the function $\delta_{P}^{-\frac{1}{2}} W(m g)$ on $M$ belongs to $W^{\psi_{N_{\mathrm{M}}}}(\pi)$. For any $s \in \mathbb{C}$ we have a representation $\operatorname{Ind}\left(W^{\psi_{N_{\mathbb{M}}}}(\pi), s\right)$ on the space $\operatorname{Ind}\left(W^{\psi_{N_{\mathbb{M}}}}(\pi)\right)$ given by $(I(s, g) W)_{s}(x)=W_{s}(x g), x, g \in G$. It is equivalent to the induced representation of $\pi \otimes \nu^{s}$ from $P$ to $G$. The family $W_{s}, s \in \mathbb{C}$ is a holomorphic section of this family of induced representations.

For any $W \in C^{\mathrm{sm}}\left(N \backslash B, \psi_{N}\right)$ and $\Phi \in \mathcal{S}\left(E^{n}\right)$, we define a function on $G^{\prime}$ by

$$
A^{\psi, \Upsilon}(W, \Phi, g, s):=\int_{V_{\gamma} \backslash V} W_{s}(\gamma v g) \omega_{\psi_{N_{\mathbb{M}}}^{-1}}^{\Upsilon_{1}^{-1}}(v g) \Phi\left(\xi_{n}\right) d v, \quad g \in G^{\prime} .
$$

where with $\xi_{n}, \gamma, V$ and $V_{\gamma}$ are defined in 2.1 Its property was studied by [29, Lemma 5.1]. In particular, it satisfies

$$
A^{\psi, \Upsilon}\left(I(s, v x) W, \omega_{\psi_{N_{\mathbb{M}}}^{-1}}^{\Upsilon^{-1}}(v x) \Phi, g, s\right)=A^{\psi, \Upsilon}(W, \Phi, g x, s)
$$


for any $g, x \in G^{\prime}$ and $v \in V$ (see Lapid-Mao [29, (5.2)]). Further, the integrand of the definition is compactly supported, and $A^{\psi, \Upsilon}$ gives rise to a $V \ltimes G$-intertwining map

$$
A^{\psi, \Upsilon}: C^{s m}\left(N \backslash G, \psi_{N}\right) \otimes \mathcal{S}\left(E^{n}\right) \rightarrow C^{s m}\left(N^{\prime} \backslash G_{\circ}, \psi_{N_{\circ}}\right)
$$

where $V \ltimes G_{\circ}$ acts via $V \ltimes \eta\left(G_{\circ}\right)$ by right translation on $C^{s m}\left(N^{\prime} \backslash G_{\circ}, \psi_{N^{\prime}}\right)$.

In order to simplify the notation, we introduce the map $A_{\#}^{\psi, \Upsilon}$ as follows. Let $V_{+} \subset V$ be the image under $\ell_{M}$ of the space of $\mathbf{n} \times \mathbf{n}$-matrices whose rows are zero except possibly for the last one. For $c=\ell_{M}(x) \in V_{+}$we denote by $\underline{c} \in E^{n}$ the last row of $x$. Then we have

$$
A^{\psi, \Upsilon}(W(\cdot c), \Phi(\cdot+\underline{s}), g)=A^{\psi, \Upsilon}(W, \Phi, g), \quad c \in V_{+}, g \in G^{\prime} .
$$

This implies that $A^{\psi, \Upsilon}(W, \Phi, \cdot)$ factors through $W \otimes \Phi \mapsto \Phi * W$ where for any function $f \in C^{\infty}(G)$ we set

$$
\Phi * f(g)=\int_{V_{+}} f(g c) \Phi(\underline{c}) d c .
$$

Then we define the map

by

$$
A_{\#}^{\psi, \Upsilon}: C^{\mathrm{sm}}\left(N \backslash G, \psi_{N}\right) \rightarrow C^{\mathrm{sm}}\left(N^{\prime} \backslash G^{\prime}, \psi_{N}\right) .
$$

$$
A_{\#}^{\psi, \Upsilon}(W, \cdot)=A^{\psi, \Upsilon}(W, \Phi, \cdot)
$$

for any $\Phi \in \mathcal{S}\left(E^{n}\right)$ such that $\Phi * W=W$. Then $A_{\#}^{\psi, \Upsilon}$ does not have the invariance by $G^{\prime}$ and $V$, but it has the following equivariance property. Set

$$
\psi_{V_{-}}(v u)=\psi_{N_{M}}(v)^{-1} \psi_{U}(u)
$$

Recall that $\psi_{U}(\ell(x))=\psi\left(\frac{1}{2}\left(x_{n, n+1}-x_{2 n, 1}\right)\right)$

Lemma 3.1 (cf. Lemma 3.1 in [30]). For any $v \in V_{-}, p=\varrho^{\prime}(g) u \in P^{\prime}$ where $g \in \mathbb{M}^{\prime}$ and $u \in U^{\prime}$, we have

$$
A_{\#}^{\psi, \Upsilon}(W(\cdot v \eta(p)), g)=\nu^{\prime}(m)^{\frac{1}{2}} \Upsilon_{M^{\prime}}(m) \alpha_{\psi^{-1}}(m)^{-1} \psi_{V_{-}}(v) A_{\#}^{\psi, \Upsilon}(W, g p)
$$

Proof. This is proved in the same argument as the proof of [30, Lemma 3.1]. Indeed, for $\Phi \in \mathcal{S}\left(E^{n}\right)$ with sufficiently small support and $\int_{E^{n}} \Phi(x) d x=1$, we can show that by (2.4), (2.5), (2.8), (2.9),

$$
\Phi^{\prime} * W(\cdot v p)=\psi_{V_{-}}(v)^{-1} \alpha_{\psi^{-1}}(m) \nu^{\prime}(m)^{-\frac{1}{2}} \Upsilon_{M^{\prime}}(m)^{-1} W(\cdot v p) .
$$

where $\Phi^{\prime}=\omega_{\psi^{-1}}^{\Upsilon^{-1}}(v p) \Phi$. This identity shows that

$$
A^{\psi, \Upsilon}\left(W(\cdot v \eta(p)), \Phi^{\prime}, g\right)=\nu^{\prime}(m)^{-\frac{1}{2}} \Upsilon_{M^{\prime}}(m)^{-1} \alpha_{\psi^{-1}}(m) \psi_{V_{-}}(v)^{-1} A_{\#}^{\psi, \Upsilon}(W(\cdot v \eta(p)), g) .
$$

Further, from the definition, we have

$$
A^{\psi, \Upsilon}\left(W(\cdot v \eta(p)), \Phi^{\prime}, g\right)=A^{\psi, \Upsilon}(W, \Phi, g p)
$$

and the lemma follows from these two identities.

As in [30, Lemma 3.2], we record the following result, which is proved as in the proof of [31, Lemma 4.5] (See also [29, Lemma 5.1]).

Lemma 3.2. For any $K_{0} \in \mathcal{C} \mathcal{S G R}(G)$, there exists $\Omega \in \mathcal{C} \mathcal{S G R}\left(V_{U}\right)$ such that for any $W \in C\left(N \backslash G, \psi_{N}\right)^{K_{0}}$ the support of $W(\gamma \cdot)_{V_{-}}$is contained in $V_{\gamma} \eta\left(w_{U^{\prime}}^{\prime}\right) \Omega \eta\left(w_{U^{\prime}}^{\prime}\right)^{-1}$.

3.2. Explicit local descent. Define the intertwining operator

$$
M(\pi, s)=M(s): \text { Ind }\left(\mathbb{W}^{\psi_{N_{M}}}(\pi), s\right) \rightarrow \text { Ind }\left(\mathbb{W}^{\psi_{N_{M}}}\left(\pi^{\vee}\right),-s\right)
$$

by (the analytic continuation of)

$$
M(s) W(g)=\nu(g)^{s} \int_{U} W_{s}\left(\varrho(\mathfrak{t}) w_{U} u g\right) d u
$$

where $\mathfrak{t}=\operatorname{diag}(1,-1, \ldots, 1,-1)$ is introduced in order to preserve the character $\psi_{N_{M}}$.

$$
w_{U}=\left(\begin{array}{cc}
1_{2 n} \\
-1_{2 n}
\end{array}\right) \text {. }
$$

Then we know that $M(s)$ is holomorphic at $s=\frac{1}{2}$ when $\pi \in \operatorname{Irr}_{\text {gen }} \mathbb{M}$ is of unitary type by Lapid-Mao [29, Proposition 2.1]. By abuse of notation, we will also denote by $M(\pi, s)$ the intertwining operator Ind $\left(\mathbb{W}^{\psi_{N_{M}}^{-1}}(\pi), s\right) \rightarrow \operatorname{Ind}\left(\mathbb{W}^{\psi_{N_{M}}^{-1}}\left(\pi^{\vee}\right),-s\right)$ defined in the same way. 
For simplicity we denote $M_{s}^{*} W:=(M(s) W)_{-s}$ so that

$$
M_{s}^{*} W=\int_{U} W_{s}\left(\varrho(\mathfrak{t}) w_{U} u \cdot\right) d u
$$

for $\operatorname{Re}(s) \gg_{\pi} 1$. Set $M^{*} W:=M_{\frac{1}{2}}^{*} W$.

Definition 3.1 (explicit local descent). Suppose that $\pi \in \operatorname{Irr}_{\text {gen }} \mathbb{M}$ is of unitary type. The we denote by $\mathcal{D}_{\psi}^{\Upsilon}(\pi)$ the space of Whittaker function on $G^{\prime}$ generated by

$$
A^{\psi, \Upsilon}\left(M\left(\frac{1}{2}\right) W, \Phi, \cdot-\frac{1}{2}\right), \quad W \in \operatorname{Ind}\left(\mathbb{W}^{\psi_{N_{M}}}(\pi)\right), \Phi \in \mathcal{S}\left(E^{n}\right)
$$

We note that from [29, Proposition 2.1] and the remark below [29, Lemma 5.1], $\mathcal{D}_{\psi}^{\Upsilon}(\pi)$ is not zero.

3.3. Good representations. Let $\pi \in \operatorname{Irr}_{\text {gen }} \mathbb{M}$ and $\sigma \in \operatorname{Irr}_{\psi_{\text {gen, }}^{-1}} G^{\prime}$ with the Whittaker model $\mathbb{W}^{N_{N^{\prime}}}(\sigma)$. For any $W^{\prime} \in \mathbb{W}^{\psi_{N^{\prime}}^{-1}}(\sigma)$ and $W \in \operatorname{Ind}\left(\mathbb{W}^{\psi_{N_{M}}}(\pi)\right)$, we define

$$
J\left(W^{\prime}, W, s\right):=\int_{N^{\prime} \backslash G^{\prime}} W^{\prime}\left(g^{\prime}\right) A_{\#}^{\psi, \Upsilon}\left(W_{s}, g^{\prime}\right) d g^{\prime} .
$$

By [6. Proposition 3.1], $J$ converges for $\operatorname{Re}(s) \gg_{\pi, \sigma} 1$ and admits a meromorphic continuation in $s$. Moreover, by 6. Proposition 4.1], for any $s \in \mathbb{C}$, we can choose $W$ and $W^{\prime}$ such that $J\left(W^{\prime}, W, s\right) \neq 0$.

Definition 3.2. Let $\pi \in \operatorname{Irr}_{\text {gen,ut }} \mathbb{M}$. We say $\pi$ is good if it satisfies the following three assumptions.

(1) $\mathcal{D}_{\psi}^{\Upsilon}(\pi)$ and $\mathcal{D}_{\psi^{-1}}^{\Upsilon^{-1}}(\mathfrak{c}(\pi))$ are irreducible.

(2) $J\left(W^{\prime}, W, s\right)$ is holomorphic at $s=\frac{1}{2}$ for any $W^{\prime} \in \mathcal{D}_{\psi^{-1}}^{\Upsilon^{-1}}(\mathfrak{c}(\pi))$ and $W \in \operatorname{Ind}\left(\mathbb{W} \psi_{N_{M}}(\pi)\right)$.

(3) There exists a non-degenerate $G$-invariant bilinear form $[\cdot, \cdot]_{\sigma}$ on $\mathcal{D}_{\psi}^{\Upsilon}(\pi) \times \mathcal{D}_{\psi^{-1}}^{\Upsilon-1}(\mathfrak{c}(\pi))$ such that

$$
J\left(W^{\prime}, W, \frac{1}{2}\right)=\left[W^{\prime}, A_{\#}^{\psi, \Upsilon}\left(M^{*} W, \cdot\right)\right]_{\sigma}
$$

Good representations appear as a local component of certain automorphic representations as follows. Let $F$ be a number field and $E$ a quadratic extension of $F$. For an irreducible cuspidal automorphic representation $\pi$ of $\mathrm{GL}_{m}\left(\mathbb{A}_{E}\right)$, whose central character is trivial on $\mathbb{A}_{F}^{\times}$, we say that $\pi$ is of unitary type if

$$
\int_{\mathbb{A}_{F}^{\times} \mathrm{GL}_{m}(F) \backslash \mathrm{GL}_{m}\left(\mathbb{A}_{F}\right)} \varphi(h) d h \neq 0
$$

for some $\varphi \in \pi$. We write $\operatorname{Cusp}_{\text {uni }} \mathrm{GL}_{m}$ for the set of irreducible cuspidal automoprhic representations of unitary type. Consider the set UCuspM of isobaric automorphic representations $\pi_{1} \boxplus \cdots \boxplus \pi_{k}$ of pairwise inequivalent $\pi_{i} \in \mathrm{Cusp}_{\mathrm{uni}} \mathrm{GL}_{n_{i}}$ for $1 \leq i \leq k$ and $n_{1}+\cdots n_{k}=2 n$. Then we have the following crucial fact.

Theorem 3.1 (Theorem 5.5 in [29]). If $\pi \in \mathrm{UCuspM}$, then its local component $\pi_{v}$ are good.

Remark 3.1. In 29, Theorem 5.5], they assume two working assumptions 4.2 and 5.2. The working assumption 5.2 is proved by Ben-Artzi and Soudry [6] and the working assumption 4.2 is proved by the author [43].

The definition of good representations was introduced and discussed in [29, 5.3]. In particular, if $\pi$ is good and $\sigma^{\prime}=\mathcal{D}_{\psi^{-1}}^{\Upsilon^{-1}}(\pi)$, then there exists a constant $c_{\pi}$ such that for any $W^{\prime}$

$$
\int_{N^{\prime}}^{s t}\left[\sigma(n) W^{\prime},\left(W^{\prime}\right)^{\vee}\right]_{\sigma} \psi_{N^{\prime}}(n) d n=c_{\pi} W^{\prime}(e)\left(W^{\prime}\right)^{\vee}(e) .
$$

More explicitly, for any $W \in \operatorname{Ind}\left(\mathbb{W}^{\psi_{N_{M}}}(\pi)\right)$ and $W^{\wedge} \in \operatorname{Ind}\left(\mathbb{W}^{\psi_{N_{M}}^{-1}}(\pi)\right.$ we have

$$
\int_{N^{\prime}}^{s t} J\left(A_{\#}^{\psi^{-1}, \Upsilon^{-1}}\left(M^{*} W^{\wedge}, \cdot n\right), W, \frac{1}{2}\right) \psi_{N^{\prime}}(n) d n=c_{\pi} A_{\#}^{\psi^{-1}, \Upsilon^{-1}}\left(M^{*} W^{\wedge}, e\right) A_{\#}^{\psi, \Upsilon}\left(M^{*} W, e\right) .
$$

In the rest of the paper we will prove the following statement:

Theorem 3.2. For any unitarizable $\pi \in \operatorname{Irr}_{\text {gen,ut }} \mathbb{M}$ which is good we have $c_{\pi}=\omega_{\pi}(\tau)$.

Remark 3.2. As remarked in Introduction, from the definition, we have $\omega_{\pi}(\tau)=\omega_{\sigma}(-1)$.

The following special case for our application to formal degree conjecture. 
Corollary 3.1. Let $\pi$ be an irreducible representation of $\mathrm{GL}_{2 n}(E)$ of the form $\pi=\tau_{1} \times \cdots \times \tau_{k}$ where $\tau_{i}$ are mutually inequivalent irreducible discrete series representations of $\mathrm{GL}_{n_{i}}(E)$ such that $n=n_{1}+\cdots+n_{k}$ and $L\left(s, \tau_{i}\right.$, As) has a pole at $s=0$. Then $\pi$ is good and $\sigma=\mathcal{D}_{\psi^{-1}}^{\Upsilon^{-1}}(\mathfrak{c}(\pi))$ is discrete series. Moreover,

$$
\int_{N^{\prime}}^{s t} J\left(W, W^{\prime}, \frac{1}{2}\right) \psi_{N^{\prime}}(n) d n=\omega_{\sigma}(-1) W(e) A_{\#}^{\psi, \Upsilon}\left(M^{*} W^{\prime}, e\right)
$$

for any $W \in \mathbb{W}^{\psi_{N^{\prime}}^{-1}}(\sigma)$ and $W^{\prime} \in \operatorname{Ind}\left(\mathbb{W}^{\psi_{N_{M}}}(\pi)\right)$.

Proof. By Theorem A.5, we find that $\sigma$ is discrete series representation. Moreover, by [20, Corollary A.6] (see also proof of Theorem A.5, we see that $\pi$ is good. Then our assertion follows from Theorem 3.2

3.4. A reduction to tempered case. In this section, we shall reduce Theorem 1.2 to the case of tempered good representations. For our reduction, we shall need lemma concerning analytic properties of local integrals. In the same argument as the proof of [31, Lemma 4.6], we can prove the following lemma using Soudry-Tanay [50, Proposition 2.1]

Lemma 3.3. Suppose that $\pi$ is an irreducible generic admissible representation of $\mathbb{M}$ and $\alpha \in \mathbb{R}$ with $\alpha>e(\pi)$. Then for any $W \in \operatorname{Ind}\left(\mathbb{W}_{N_{M}}(\pi)\right)$ and $\Phi \in \mathcal{S}\left(E^{n}\right)$, there exists $c>0$ such that for any $t^{\prime}=\varrho^{\prime}(t) \in T^{\prime}$ with $t=\left(t_{1}, \ldots, t_{n}\right) \in \mathbb{M}^{\prime}$, $k \in K^{\prime}$ and $s \in \mathbb{C}$, we have $A^{\psi, \Upsilon}\left(W, \Phi, t k^{\prime}, s\right)=0$ unless $\left|t_{i}\right| \leq c\left|t_{i+1}\right|$ for $i=1, \ldots, n$ with $t_{n+1}=1$ in which case

$$
\left|A^{\psi, \Upsilon}\left(W, \Phi, \varrho^{\prime}(t) k^{\prime}, s\right)\right| \ll \delta_{B^{\prime}}\left(t^{\prime}\right)^{\frac{1}{2}}|\operatorname{det}(t)|^{\operatorname{Re}(s)+\frac{1}{2}-\alpha} .
$$

Similarly as the proof of [31, Lemma 4.12], the following lemma readily follows from the above lemma.

Lemma 3.4. Suppose that $\sigma \in \operatorname{Irr}_{\psi_{N^{\prime}}^{-1} \text {,gen }} G^{\prime}$ is essentially discrete series representation and $\alpha=e(\pi)$. Then there exists $\delta>0$ such that $J\left(W^{\prime}, W, \Phi, s\right)$ is absolutely convergent locally uniformly (hence, holomorphic) for $\operatorname{Re}(s) \geq \alpha-\delta-\frac{1}{2}$ where $W^{\prime} \in \mathbb{W}^{\psi_{N^{\prime}}^{-1}}(\sigma), W \in \operatorname{Ind}\left(\mathbb{W}^{\psi_{N_{M}}}(\pi)\right)$ and $\Phi \in \mathcal{S}\left(E^{n}\right)$. Similarly, for any $W \in \operatorname{Ind}\left(\mathbb{W}^{\psi_{N_{M}}}(\pi)\right), W^{\vee} \in \operatorname{Ind}\left(\mathbb{W}^{\psi_{N_{M}}^{-1}}(\pi)\right)$, $\Phi, \Phi^{\vee} \in \mathcal{S}\left(E^{n}\right)$,

$$
J\left(A^{\psi^{-1}, \Upsilon^{-1}}\left(M\left(\frac{1}{2}\right) \mathfrak{c}\left(W^{\vee}\right), \Phi^{\vee}, \cdot,-\frac{1}{2}\right), W, \Phi, s\right)
$$

converges absolutely and locally uniformly (and hence, is holomorphic) for Res $>2 \alpha-\frac{1}{2}$. In particular, if $\pi$ is unitarizable then $J\left(W^{\prime}, W, \Phi, s\right)$ is holomorphic at $s=\frac{1}{2}$ for any $W^{\prime} \in \mathcal{D}_{\psi^{-1}}(\pi), W \in \operatorname{Ind}\left(\mathbb{W}^{\psi_{N_{M}}}(\pi)\right)$ and $\Phi \in \mathcal{S}\left(E^{n}\right)$.

When the base field $F$ is real field, we may prove similar results in the same argument as the proof of [29, Lemma 4.11]. For later use, we would like to state them here.

Lemma 3.5. Suppose that $\pi$ is an irreducible generic admissible representation of $\mathbb{M}$ and $\alpha \in \mathbb{R}$ with $\alpha>e(\pi)$. Then for any $W \in \operatorname{Ind}\left(\mathbb{W}^{\psi_{N_{M}}}(\pi)\right)$ and $\Phi \in \mathcal{S}\left(E^{n}\right)$, there exists a compact set $D \subset \mathbb{C}$ and $m \geq 1$ we have

$$
\left|A^{\psi, \Upsilon}\left(W, \Phi, \varrho^{\prime}(t) k^{\prime}, s\right)\right| \ll \delta_{B^{\prime}}\left(t^{\prime}\right)^{\frac{1}{2}}|\operatorname{det}(t)|^{\operatorname{Re}(s)+\frac{1}{2}-\alpha}\left(1+\left|t_{i} t_{i+1}^{-1}\right|\right)^{-m}
$$

for any $t^{\prime}=\varrho^{\prime}(t) \in T^{\prime}$ with $t=\left(t_{1}, \ldots, t_{n}\right) \in \mathbb{M}^{\prime}, k \in K^{\prime}$ and $s \in D$.

Remark 3.3. In the same argument as the proof of [29, Lemma 4.12] with Lemma 3.5, Lemma 3.4 holds over real field.

Let us go back to our situation, namely we suppose that the base field is non-arhchimedean local field. Then our aim in this section is to prove the following reduction.

Proposition 3.1. Suppose that (1.1) holds for any good $\pi \in \operatorname{Irr}_{\mathrm{temp}, \mathrm{ut}} \mathbb{M}$ such that $\mathcal{D}_{\psi^{-1}}^{\Upsilon^{-1}}(\pi)$ is tempered. Then Theorem 3.2 holds.

For a proof, recall the following classification of generic representations of unitary type by Matringe.

Theorem 3.3 (Theorem 5.2 in Matringe [37). The set $\operatorname{Irr}_{\mathrm{gen}, \mathrm{ut}} \mathbb{M}$ consists of the irreducible representations of the form

$$
\pi=\mathfrak{c}\left(\sigma_{1}\right) \times \sigma_{1}^{\vee} \times \cdots \mathfrak{c}\left(\sigma_{k}\right) \times \sigma_{k}^{\vee} \times \delta_{1} \times \cdots \times \delta_{l}
$$

where $\sigma_{1}, \ldots, \sigma_{k}$ are essentially square-integrable representations, and $\delta_{1}, \ldots, \delta_{l}$ are square-integrable representations of unitary type (i.e., $L\left(0, \tau_{i}, \mathrm{As}\right)=\infty$ for all $i$ ). Here, $\times$ denotes the parabolic induction of $\mathrm{GL}_{m}$.

We shall prove the reduction following the argument in [29, 3.6].

Proof. Let $\pi \in \operatorname{Irr}_{\text {gen,ut }} \mathbb{M}$ be unitarizable and good. Then by Theorem 3.3, we may write

$$
\pi=\mathfrak{c}\left(\sigma_{1}\right)\left[a_{1}\right] \times \sigma_{1}^{\vee}\left[-a_{1}\right] \times \cdots \times \mathfrak{c}\left(\sigma_{k}\right)\left[a_{k}\right] \times \sigma_{k}^{\vee}\left[-a_{k}\right] \times \delta_{1} \times \cdots \times \delta_{l}
$$

where $\sigma_{i} \in \operatorname{Irr}_{\mathrm{sqr}} \mathrm{GL}_{n_{i}}(E), \delta_{i} \in \operatorname{Irr}_{\mathrm{sqr}} \mathrm{GL}_{m_{j}}(E)$ and $\left(a_{1}, \ldots, a_{k}\right) \in \mathbb{C}^{k}$ is in the domain

$$
\mathfrak{D}=\left\{\left(s_{1}, \ldots, s_{k}\right) \in \mathbb{C}^{k}:-\frac{1}{2}<\operatorname{Re} s_{i}<\frac{1}{2} \text { for all } i\right\} \text {. }
$$


Here, we write $\sigma[s]$ for the twist of $\sigma \in \operatorname{IrrGL}_{m}(E)$ by $|\operatorname{det}|^{s}$ with $s \in \mathbb{C}$. For $\underline{s}=\left(s_{1}, \ldots, s_{k}\right) \in \mathbb{C}^{k}$, let us consider the following representation

$$
\pi(\underline{s}):=\mathfrak{c}\left(\sigma_{1}\right)\left[s_{1}\right] \times \sigma_{1}^{\vee}\left[-s_{1}\right] \times \cdots \times \mathfrak{c}\left(\sigma_{k}\right)\left[s_{k}\right] \times \sigma_{k}^{\vee}\left[-s_{k}\right] \times \delta_{1} \times \cdots \times \delta_{l}
$$

which is irreducible for $\underline{s} \in \mathfrak{D}$ by Zelevinsky [ 54 , Theorem 9.7].

Take a quadratic extension of number fields $K / L$ such that for some finite place $v_{0}, L_{v_{0}}=F$ and $K_{v_{0}}=E$. Let $\rho \in \operatorname{Irr}_{\text {sqr, gen }} \mathrm{U}_{\mathbf{m}}$ be the representation corresponding to $\delta_{1} \times \cdots \times \delta_{l}$ under the correspondence established in Theorem A.4 with $\mathbf{m}=m_{1}+\cdots+m_{l}$. Then we put

$$
\sigma(\underline{s}):=\mathfrak{c}\left(\sigma_{1}\right)\left[s_{1}\right] \times \sigma_{1}^{\vee}\left[-s_{1}\right] \times \cdots \times \mathfrak{c}\left(\sigma_{k}\right)\left[s_{k}\right] \times \sigma_{k}^{\vee}\left[-s_{k}\right] \rtimes \rho
$$

By Konno [27, Corollary 4.3], for a dense open subset of $\underline{s} \in i \mathbb{R}^{k}, \sigma(\underline{s}) \in \operatorname{Irr}_{\text {temp }} \mathrm{U}_{2 n}$. Further, by [20, Corollary A.8], for a dense open subset of $\underline{s} \in i \mathbb{R}^{k}, \sigma(\underline{s})$ is a local component of an irreducible cuspidal automorphic representation of $\mathrm{U}_{2 n}\left(\mathbb{A}_{L}\right)$. From our proof of Theorem $\mathrm{A}$.4 we find that its base change lift to $\mathrm{GL}_{2 n}\left(\mathbb{A}_{K}\right)$ by Kim-Krishnamurthy [25] gives a globalization of $\pi(\underline{s})$, and $\mathcal{D}_{\psi}^{\Upsilon^{-1}}(\pi(\underline{s}))=\sigma(\underline{s})$. In particular, by [29. Theorem 5.5], $\pi(\underline{s})$ is good for such $\underline{s}$. Further, it is tempered since $\underline{s} \in i \mathbb{R}^{k}$. We note that $\omega_{\pi(\underline{s})}(\tau)=\omega_{\tau_{1}}(\tau) \cdots \omega_{\tau_{l}}(\tau)=\omega_{\pi}(\tau)$. Hence, from our assumption, for $\underline{s} \in i \mathbb{R}^{k}$ given in the above discussion, we have

$$
c_{\pi(\underline{s})}=\omega_{\pi(\underline{s})}(\tau)=\omega_{\pi}(\tau)
$$

On the other hand, in the same argument as [33, 3.6], Lemma imply that both sides of are holomorphic functions of $\underline{s} \in \mathfrak{D}$. This shows that $c_{\pi(\underline{s})}$ is a meromorphic function on $\underline{s} \in \mathfrak{D}$, and thus $c_{\pi(\underline{s})}$ is a constant function of $\underline{s}$ by (3.4). In particular, we have

$$
c_{\pi}=\omega_{\pi}(\tau)
$$

In the reminder of the paper we will prove Theorem 3.2, i.e., the Main Identity

$$
\left.\int_{N^{\prime}}^{s t}\left(\int_{N^{\prime} \backslash G^{\prime}} A_{\#}^{\psi, \Upsilon}\left(W_{s}, g\right) A_{\#}^{\psi^{-1}, \Upsilon^{-1}}\left(M^{*} W^{\wedge}, g u\right) d g\right) \psi_{N^{\prime}}(u) d u\right|_{s=\frac{1}{2}}=\omega_{\pi}(\tau) A_{\#}^{\psi^{-1}, \Upsilon^{-1}}\left(M^{*} W^{\wedge}, e\right) A_{\#}^{\psi, \Upsilon}\left(M^{*} W, e\right)
$$

under the assumptions that $\pi \in \operatorname{Irr}_{\text {temp,ut }} \mathbb{M}$ is good and $\sigma:=\mathcal{D}_{\psi^{-1}}^{\Upsilon^{-1}}(\mathfrak{c}(\pi))$ is tempered.

\section{A BILINEAR FORM}

Following [29, we shall study the main identity. A key ingredient of this section is the stability of the integral defining a Bessel functions, which is given in Appendix B. Indeed, we apply Theorem B.2 to the function $A_{\#}^{\psi, \Upsilon}(W, g)$ (See also Remark B.1).

Proposition 4.1. Let $K_{0} \in \mathcal{C} \mathcal{S G R}(G)$. Then the integral

$$
Y^{\psi, \Upsilon}(W, t):=\int_{N^{\prime}}^{s t} A_{\#}^{\psi, \Upsilon}\left(W, w_{U^{\prime}}^{\prime} w_{0}^{M^{\prime}} t n\right) \psi_{N^{\prime}}(n)^{-1} d n, \quad t \in T^{\prime}
$$

stabilizes uniformly for $W \in C\left(N \backslash G, \psi_{N}\right)^{K_{0}}$ and locally uniformly in $t \in T^{\prime}$. In particular, $Y^{\psi, \Upsilon}\left(W_{s}, t\right)$ is entire in $s \in \mathbb{C}$ and if $\pi \in \operatorname{Irr}_{g e n} \mathbb{M}$ and $W \in \operatorname{Ind}\left(\mathbb{W}^{\psi_{N_{M}}}(\pi)\right)$ then $Y^{\psi, \Upsilon}\left(M_{s}^{*} W, t\right)$ is meromorphic in s. Both $Y^{\psi, \Upsilon}\left(W_{s}, t\right)$ and $Y^{\psi, \Upsilon}\left(M_{s}^{*} W, t\right)$ are locally constant in $t$, uniformly in $s \in \mathbb{C}$.

Finally, if we assume that $\pi \in \operatorname{Irr}_{\text {meta,gen }} \mathbb{M}$ and that $\pi_{\circ}=\mathcal{D}_{\psi^{-1}}^{\Upsilon^{-1}}(\pi)$ is irreducible then for any $W^{\wedge} \in \operatorname{Ind}\left(\mathbb{W}^{\psi_{N_{M}}^{-1}}(\pi)\right)$ we have

$$
Y^{\psi^{-1}, \Upsilon^{-1}}\left(M^{*} W^{\wedge}, t\right)=\mathbb{B}_{\pi_{\circ}}^{\psi_{N_{\circ}}^{-1}}\left(w_{U^{\prime}}^{\prime} w_{0}^{M^{\prime}} t\right) A_{\#}^{\psi^{-1}, \Upsilon^{-1}}\left(M^{*} W^{\wedge}, e\right)
$$

We use Theorem B.2 in another way as follows, which is proved in the same argument as [30, Lemma 5.4].

Lemma 4.1. Let $W^{\prime} \in C^{s m}\left(N^{\prime} \backslash G^{\prime}, \psi_{N^{\prime}}\right)$ and $\left(W^{\prime}\right)^{\wedge} \in C^{s m}\left(N^{\prime} \backslash G^{\prime}, \psi_{N^{\prime}}^{-1}\right)$. Assume that the function $(t, n) \in T^{\prime} \times N^{\prime} \mapsto$ $W^{\prime}\left(w_{0}^{\prime} t n\right)$ is compactly supported. (In particular, $W^{\prime} \in C_{c}^{s m}\left(N^{\prime} \backslash G^{\prime}, \psi_{N^{\prime}}\right)$.) Then the iterated integral

$$
\int_{N^{\prime}}^{s t}\left(\int_{N^{\prime} \backslash G^{\prime}} W^{\prime}(g)\left(W^{\prime}\right)^{\wedge}(g u) d g\right) \psi_{N^{\prime}}(u) d u
$$

is well-defined and is equal to

$$
\int_{T^{\prime}}\left(\int_{N^{\prime}} \delta_{B^{\prime}}(t) W^{\prime}\left(w_{U^{\prime}}^{\prime} w_{0}^{M^{\prime}} t n\right) \psi_{N^{\prime}}\left(n^{-1}\right) d n\right)\left(\int_{N^{\prime}}^{s t}\left(W^{\prime}\right)^{\wedge}\left(w_{U^{\prime}}^{\prime} w_{0}^{M^{\prime}} t u\right) \psi_{N^{\prime}}(u) d u\right) d t .
$$


4.1. In order to apply Lemma 4.1 for $A_{\#}^{\psi, \Upsilon}\left(W_{s}, \cdot\right)$ we make a special choice of $W$. Consider the $P$-invariant subspace $\operatorname{Ind}\left(\mathbb{W}_{N_{M}}(\pi)\right)^{\circ}$ of $\operatorname{Ind}\left(\mathbb{W}^{\psi_{N_{M}}}(\pi)\right)$ consisting of functions supported in the big cell $P w_{U} P=P w_{U} U$. Any element of $\operatorname{Ind}\left(\mathbb{W} \psi_{N_{M}}(\pi)\right)^{\circ}$ is a linear combination of functions of the form

$$
W\left(u^{\prime} m w_{U} u\right) \delta_{P}(m)^{\frac{1}{2}} W^{M}(m) \phi(u), m \in M, u, u^{\prime} \in U
$$

with $W^{M} \in \mathbb{W}^{\psi_{N_{M}}}(\pi)$ and $\phi \in C_{c}^{\infty}(U)$. Let $\eta_{\mathbb{M}}$ be the embedding $\eta_{\mathbb{M}}(g)=\left({ }^{g}{ }_{I_{n}}\right)$ of $\mathbb{M}^{\prime}$ into $\mathbb{M}$. Also let $\eta_{M}=\varrho \circ \eta_{\mathbb{M}}$ so that $\eta_{M}(g)=\left(\begin{array}{ccc}g & & \\ & I_{2 n} & \\ & & g^{*}\end{array}\right)$

Definition 4.1. Let $\operatorname{Ind}\left(\mathbb{W}^{\psi_{N_{M}}}(\pi)\right)_{\#}^{\circ}$ be the linear subspace of $\operatorname{Ind}\left(\mathbb{W} \psi_{N_{M}}(\pi)\right)$ generated by $W^{s}$ as in (4.1) that satisfy the additional property that the function $(t, n) \mapsto W^{M}\left(\eta_{M}\left(t w_{0}^{\mathbb{M}^{\prime}} n\right)\right)$ is compactly supported on $T_{\mathbb{M}^{\prime}}^{\prime} \times N_{\mathbb{M}^{\prime}}^{\prime}$, or equivalently, that the function $W^{M} \circ \eta_{M}$ on $\mathbb{M}^{\prime}$ is supported in the big cell $B_{\mathbb{M}^{\prime}}^{\prime} w_{0}^{\mathbb{M}^{\prime}} N_{\mathbb{M}^{\prime}}^{\prime}$ and its support is compact module $N_{\mathbb{M}^{\prime}}^{\prime}$.

Note that this space is non-zero by the proof of [30, Lemma 6.13].Further, this space is invariant under $\eta\left(T^{\prime}\right) \ltimes N$.

Lemma 4.2. For any $W \in \operatorname{Ind}\left(\mathbb{W}^{\psi_{N_{M}}}(\pi)\right)_{\#}^{\circ}$, the function $A_{\#}^{\psi, \Upsilon}\left(W_{s}, w_{U^{\prime}}^{\prime} w_{0}^{M^{\prime}} t n\right)$ is compactly supported in $t \in T^{\prime}$ and $n \in N^{\prime}$ uniformly in $s \in \mathbb{C}$.

Proof. A proof is identical to the proof of [30, Lemma 5.6].

4.2. For $W \in \operatorname{Ind}\left(\mathbb{W}^{\psi_{N_{M}}}(\pi)\right)_{\#}^{\circ}$ and $W^{\vee} \in \operatorname{Ind}\left(\mathbb{W}^{\psi_{N_{M}}^{-1}}\left(\pi^{\vee}\right)\right)$, we define

$$
B\left(W, W^{\vee}, s\right):=\int_{N^{\prime}}^{s t} \int_{N^{\prime} \backslash G^{\prime}} A_{\#}^{\psi, \Upsilon}\left(W_{s}, g\right) A_{\#}^{\psi^{-1}, \Upsilon^{-1}}\left(W_{-s}^{\vee}, g u\right) \psi_{N^{\prime}}(u) d u .
$$

By Lemma 4.2, we may apply the argument in the proof of [30, Lemma 5.4]. Then we have

$$
\int_{T^{\prime}} \int_{N^{\prime}} \int_{\Omega} \delta_{B^{\prime}}(t) A_{\#}^{\psi, \Upsilon}\left(W_{s}, w_{U^{\prime}}^{\prime} w_{0}^{M^{\prime}} t n\right) A_{\#}^{\psi^{-1}, \Upsilon^{-1}}\left(W_{-s}^{\vee}, w_{U^{\prime}}^{\prime} w_{0}^{M^{\prime}} \text { tnu }\right) \psi_{N^{\prime}}(u) d u d n d t
$$

for any sufficiently large $\Omega \in \mathcal{C} \mathcal{S G R}\left(N^{\prime}\right)$. This implies that $B\left(W, W^{\vee}, s\right)$ is an entire function of $s$ and from Proposition 4.1 we have

$$
B\left(W, W^{\vee}, s\right)=\int_{T^{\prime}} Y^{\psi, \Upsilon}\left(W_{s}, t\right) Y^{\psi^{-1}, \Upsilon^{-1}}\left(W_{-s}^{\vee}, t\right) \delta_{B^{\prime}}(t) d t
$$

for any $W \in \operatorname{Ind}\left(\mathbb{W}^{\psi_{N_{M}}}(\pi)\right)_{\#}^{\circ}$ and $W^{\vee} \in \operatorname{Ind}\left(\mathbb{W}^{\psi_{N_{M}}^{-1}}(\pi)\right)$.

Assume that $\pi \in \operatorname{Irr}_{\text {gen,ut }} \mathbb{M}$ and $\sigma=\mathcal{D}_{\psi^{-1}}^{\Upsilon-1}(\mathfrak{c}(\pi))$ is irreducible. Then for $W \in \operatorname{Ind}\left(\mathbb{W}_{N_{M}}(\pi)\right)_{\#}^{\circ}$ and $W^{\vee} \in \operatorname{Ind}\left(\mathbb{W}^{\psi_{N_{M}}^{-1}}(\pi)\right)$,

$$
\text { the left-hand side of the Main Identity (MI) is } B\left(W, M\left(\frac{1}{2}\right) W^{\vee}, \frac{1}{2}\right)
$$

\section{FURThER ANALYSIS}

Fix an element $\varepsilon_{1}$ of the form $\ell_{M}(X)$ where $X \in \operatorname{Mat}_{\mathbf{n} \times \mathbf{n}}$ and the last row of $X$ is $-\xi_{\mathbf{n}}$. Then we can check that

$$
\psi_{V_{-}}\left(\varepsilon_{1}^{-1} v \varepsilon_{1}\right)=\psi_{V_{-}}(v) \psi\left(v_{\mathbf{n}, 2 \mathbf{n}+1}\right) .
$$

For any $W \in C^{s m}\left(N \backslash G, \psi_{N}\right)$, define

$$
A_{e}^{\psi}(W):=\int_{V_{\gamma} \backslash V_{-}} W\left(\gamma v \varepsilon_{1}\right) \psi_{V_{-}}\left(\varepsilon_{1}^{-1} v \varepsilon_{1}\right)^{-1} d v .
$$

Lemma 5.1. For any $W \in C^{\mathrm{sm}}\left(N \backslash G, \psi_{N}\right)$, the integrand in is compactly supported on $V_{\gamma} \backslash V$ and we have $A_{\#}^{\psi, \Upsilon}(W, e)=$ $A_{e}^{\psi}(W)$.

Proof. The proof is identical to the proof of [30, Lemma 6.1] because of Lemma 3.2 and (2.3)-(2.5)

Remark 5.1. The definition of $A_{\#}^{\psi, \Upsilon}(W, \cdot)$ depends on the choice of $\Upsilon$, and so does its invariance by Lemma [3.1. In this lemma, we evaluate $A_{\#}^{\psi, \Upsilon}(W, \cdot)$ only at $e$, and we see that this value is independent of the choice of $\Upsilon$. Further, from

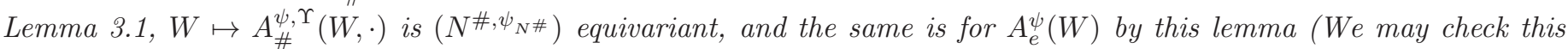
condition by a direct computation).

We now explicate $A_{\#}^{\psi, \Upsilon}\left(W_{s}, \cdot\right)$ on the big cell $N^{\prime} w_{U^{\prime}}^{\prime} P^{\prime}$. By Lemma 3.1 it is enough to consider the element $w_{U^{\prime}}^{\prime}$.

Lemma 5.2. Let $\pi \in \operatorname{Irr}_{\text {gen }} M$. Then for $\operatorname{Re} s \gg_{\pi} 1$ and $W \in \operatorname{Ind}\left(\mathbb{W} \psi_{N_{M}}(\pi)\right)$ we have

$$
A_{\#}^{\psi, \Upsilon}\left(W_{s}, w_{U^{\prime}}^{\prime}\right)=\alpha_{\psi^{-1}}\left(w_{U^{\prime}}^{\prime}\right) \int_{V_{U}} W_{s}\left(w_{U} v\right) \psi_{U}(v)^{-1} d v=\alpha_{\psi^{-1}}\left(w_{U^{\prime}}^{\prime}\right) \int_{V_{M}^{\#} \backslash V_{-}} W_{s}\left(w_{U} v\right) \psi_{V_{-}}(v)^{-1} d v \text {. }
$$


Proof. A proof is identical to the proof of [30, Lemma 6.3].

5.1. We can now explicate $Y^{\psi, \Upsilon}\left(W_{s}, t\right)$ for $\operatorname{Re} s \gg 1$.

Lemma 5.3. Let $\pi \in \operatorname{Irr}_{\text {gen }} M$. For $\operatorname{Res} \gg_{\pi} 1$ and any $W \in \operatorname{Ind}\left(\mathbb{W}^{\psi_{N_{M}}}(\pi)\right), t \in T^{\prime}$ we have the identity

$$
Y^{\psi, \Upsilon}\left(W_{s}, t\right)=\nu^{\prime}(t)^{n-\frac{1}{2}} \Upsilon_{M^{\prime}}(t)^{-1} \alpha_{\psi^{-1}}\left(w_{U^{\prime}}^{\prime}\right) \alpha_{\psi^{-1}}\left(w_{0}^{M^{\prime}} t\right) \int_{V_{M}^{\#} \backslash N \#} W_{s}\left(w_{U} \eta\left(w_{0}^{M^{\prime}} t\right) v\right) \psi_{N \#}(v)^{-1} d v
$$

where the right-hand side is absolutely convergent.

We first need the following convergence result.

Lemma 5.4 (cf. Lemma 6.8). Let $\pi \in \operatorname{Irr}_{\text {gen }} M$. Then for $\operatorname{Re} s \gg_{\pi} 1$ we have

$$
\int_{N_{\mathbb{M}}^{t} \cap \mathcal{P}} \int_{U}\left|W_{s}\left(\varrho(n) w_{U} u g\right)\right| d u d n<\infty
$$

and

$$
\int_{N_{M^{t}}^{t} \cap \mathcal{P}^{*}} \int_{U}\left|W_{s}\left(\varrho(n) w_{U} u g\right)\right| d u d n<\infty
$$

for any $W \in \operatorname{Ind}\left(\mathbb{W}^{\psi_{N_{M}}}(\pi)\right), g \in G$.

Proof. The proof of this lemma is identical to the proof of [30, Lemma 6.8].

5.2. We now go back to the bilinear form $B$. The following lemma is proved in the same argument as the proof of 30 . Lemma 6.10].

Lemma 5.5. For $W \in \operatorname{Ind}\left(\mathbb{W}^{\psi_{N_{M}}}(\pi)\right)_{\#}^{\circ}$ the integrand on the right-hand side of (5.3) is compactly supported in $t, v$ uniformly in $s$ ( $i$. e. the support in $(t, v)$ is contained in a compact set which is independent of $s)$. In particular, the identity (5.3) holds for all $s \in \mathbb{C}$.

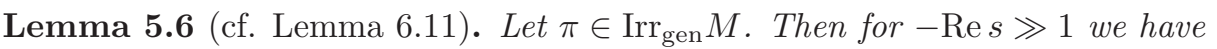

$$
B\left(W, W^{\vee}, s\right)=\int_{U} \int_{V_{M}^{\#} \backslash N \#} \int_{N_{\mathbb{M}^{\prime}} \backslash \mathbb{M}^{\prime}} W_{s}\left(\eta_{M}(g) w_{U} v\right) W_{-s}^{\vee}\left(\eta_{M}(g) w_{U} u\right) \delta_{P}\left(\eta_{M}(g)\right)^{-1}|\operatorname{det} g|^{1-n} \psi_{N^{\#}}(v)^{-1} \psi_{U}(u) d g d v d u
$$

for any $W \in \operatorname{Ind}\left(\mathbb{W}^{\psi_{N_{M}}}(\pi)\right)_{\#}^{\circ}, W^{\vee} \in \operatorname{Ind}\left(\mathbb{W}^{\psi_{N_{M}}^{-1}}\left(\pi^{\vee}\right)\right)$ with the integral being absolutely convergent.

Proof. This is proved in a similar argument as the proof of [30, Lemma 6.11] except for some minor differences. For the sake of completeness, we shall repeat their argument in our setting.

Suppose that $-\operatorname{Re} s \gg 1$. Then by (4.2), Lemma 5.3 and 5.5 and the fact

$$
\delta_{B^{\prime}}(t)=\delta_{B_{M^{\prime}}^{\prime}}(t) \nu^{\prime}(t)^{n}, \quad t \in T^{\prime} .
$$

$B\left(W, W^{\vee}, s\right)$ is equal to

$\int_{T_{\mathbb{M}^{\prime}}^{\prime}} \int_{\eta\left(N_{M^{\prime}}^{\prime}\right) \ltimes U} \int_{\eta\left(N_{M^{\prime}}^{\prime}\right) \ltimes U} W_{s}\left(\eta_{M}\left(\left(w_{0}^{\mathbb{M}^{\prime}} t\right)^{*}\right) w_{U} v_{1}\right) W_{-s}\left(\eta_{M}\left(\left(w_{0}^{\mathbb{M}^{\prime}} t\right)^{*}\right) w_{U} v_{2}\right)|\operatorname{det} t|^{3 n-1} \delta_{B_{\mathbb{M}^{\prime}}^{\prime}}(t) \psi_{N^{\#}}\left(v_{1}\right)^{-1} \psi_{N \#}\left(v_{2}\right) d v_{1} d v_{2} d t$ where the integral is absolutely convergent. By the change of variable, $B\left(W, W^{\vee}, s\right)$ is equal to

$$
\int_{T_{\mathbb{M}^{\prime}}^{\prime}} \int_{N_{\mathbb{M}^{\prime}}^{\prime}} \int_{U} \int_{\eta\left(N_{M^{\prime}}^{\prime}\right) \ltimes U} W_{s}\left(\eta_{M}\left(\left(w_{0}^{\mathbb{M}^{\prime}} t n\right)^{*}\right) w_{U} u v_{1}\right) W_{-s}\left(\eta_{M}\left(\left(w_{0}^{\mathbb{M}^{\prime}} t n\right)^{*}\right) w_{U} u\right)|\operatorname{det} t|^{3 n-1} \delta_{B_{\mathbb{M}^{\prime}}^{\prime}}(t) \psi_{N \#}\left(v_{1}\right)^{-1} d v_{1} d u d n d t .
$$

Finally, by the Bruhat decomposition and the fact

$$
\delta_{P}\left(\eta_{M}(g)\right)=|\operatorname{det} g|^{2 n}, \quad \text { for any } g \in \mathbb{M}^{\prime}
$$

the lemma readily follows.

Define when convergent

$$
\left\{W, W^{\vee}\right\}:=\int_{N_{\mathbb{M}^{\prime}}^{\prime} \backslash \mathbb{M}^{\prime}} W\left(\eta_{M}(g)\right) W^{\vee}\left(\eta_{M}(g)\right) \delta_{P}\left(\eta_{M}(g)\right)^{-1}|\operatorname{det} g|^{1-n} d g,
$$

which converges for any $\left(W, W^{\vee}\right) \in \operatorname{Ind}\left(\mathbb{W}^{\psi_{N_{M}}}(\pi)\right) \times \operatorname{Ind}\left(\mathbb{W}^{\psi_{N_{M}}^{-1}}\left(\pi^{\vee}\right)\right)$ when $\pi$ is unitarizable by [32, Lemma 1.2]. Then we get for any $W \in \operatorname{Ind}\left(\mathbb{W}^{\psi_{N_{M}}}(\pi)\right)_{\#}^{\circ}, W^{\vee} \in \operatorname{Ind}\left(\mathbb{W}^{\psi_{N_{M}}^{-1}}\left(\pi^{\vee}\right)\right)$ and when $-\operatorname{Re} s \gg 1$

$$
B\left(W, W^{\vee}, s\right)=\int_{U} \int_{V_{M}^{\#} \backslash N \#}\left\{W_{s}\left(\cdot w_{U} v\right), W_{-s}^{\vee}\left(\cdot w_{U} u\right)\right\} \psi_{N \#}(v)^{-1} \psi_{U}(v) d v d u .
$$


5.3. In the same argument as the proof of [30, Lemma 6.13] using Theorem B.3. Proposition 4.1 and Lemma 5.5 instead of [30, Theorem A.1, Corollary 5.3, Lemma 6.10], we may prove the following lemma.

Lemma 5.7. Assume that $\pi \in \operatorname{Irr}_{\text {gen,ut } M}$ and $\sigma=\mathcal{D}_{\psi^{-1}}^{\Upsilon^{-1}}(\pi)$ is irreducible and tempered. Then the bilinear form $B\left(W, M\left(\frac{1}{2}\right) W^{\wedge}, \frac{1}{2}\right)$ does not identically zero on $W \in \operatorname{Ind}\left(\mathbb{W}^{\psi_{N_{M}}}(\pi)\right)_{\#}^{\circ} \times \operatorname{Ind}\left(\mathbb{W}^{\psi_{N_{M}}^{-1}}(\mathfrak{c}(\pi))\right)$

By (4.3), Lemma 5.1 and Lemma 5.7 we conclude

Corollary 5.1. Suppose that $\pi \in \operatorname{Irr}_{\mathrm{ut}, \text { temp }} M$ is good and $\sigma=\mathcal{D}_{\psi^{-1}}^{\Upsilon^{-1}}(\pi)$ is tempered. Then

$$
B\left(W, M\left(\frac{1}{2}\right) W^{\wedge}, \frac{1}{2}\right)=c_{\pi} A_{e}^{\psi}\left(M^{*} W\right) A_{e}^{\psi^{-1}}\left(M^{*} W^{\wedge}\right)
$$

for all $W \in \operatorname{Ind}\left(\mathbb{W}^{\psi_{N_{M}}}(\pi)\right)_{\#}^{\circ}$ and $W^{\vee} \in \operatorname{Ind}\left(\mathbb{W}^{\psi_{N_{M}}^{-1}}(\mathfrak{c}(\pi))\right)$ Moreover, the linear form $A_{e}^{\psi}\left(M^{*} W\right)$ does not vanish identically on $\operatorname{Ind}\left(\mathbb{W} \psi_{N_{M}}(\pi)\right)_{\#}^{\circ}$.

In other words, because of Proposition 3.1, Theorem 3.2 is reduced to the following statement.

Proposition 5.1. Assume that $\pi \in \operatorname{Irr}_{\mathrm{ut}, \mathrm{temp}} M$ is good and $\sigma=\mathcal{D}_{\psi^{-1}}^{\Upsilon^{-1}}(\pi)$ is tempered. Then for any $W \in \operatorname{Ind}\left(\mathbb{W}^{\psi_{N_{M}}}(\pi)\right)_{\#}^{\circ}$ and $W^{\wedge} \in \operatorname{Ind}\left(\mathbb{W}^{\psi_{N}^{-1}}(\mathfrak{c}(\pi))\right)$ we have

$$
B\left(W, M\left(\frac{1}{2}\right) W^{\wedge}, \frac{1}{2}\right)=\omega_{\pi}(\tau) A_{e}^{\psi}\left(M^{*} W\right) A_{e}^{\psi^{-1}}\left(M^{*} W^{\wedge}\right)
$$

\section{Application of functional equations}

We define $\underline{B}\left(W, W^{\vee}, s\right)$ to be the right hand side of (5.4) whenever the integral defining $\{\cdot, \cdot\}$ and the double integrals over $V_{M}^{\#} \backslash N^{\#}$ and $U$ are absolutely convergent. Clearly for $g \in \mathbb{M}^{\prime}$, with $|\operatorname{det} g|=1$

$$
\left\{W\left(\cdot \eta_{M}(g)\right), W \vee\left(\cdot \eta_{M}(g)\right)\right\}=\{W, W \vee\} .
$$

Then as in [30, (7.1)], $\underline{B}\left(W, W^{\vee}, s\right)$ is equal to

$$
\begin{gathered}
\int_{N_{\mathbb{M}^{\prime}}^{\prime}} \int_{U} \int_{U}\left\{W_{s}\left(\cdot \eta_{M}(n) w_{U} v\right), W_{-s}^{\vee}\left(\cdot w_{U} u\right)\right\} \psi_{U}(v)^{-1} \psi_{U}(u) \psi_{N_{\mathbb{M}^{\prime}}^{\prime}}(n) d v d u d n d v d u d n \\
=\int_{N_{\mathbb{M}^{\prime}}^{\prime}} \int_{U} \int_{U}\left\{W_{s}\left(\cdot w_{U} v\right), W_{-s}^{\vee}\left(\cdot \eta_{M}(n) w_{U} u\right)\right\} \psi_{U}(v)^{-1} \psi_{U}(u) \psi_{N_{\mathbb{M}^{\prime}}^{\prime}}(n)^{-1} d v d u d n d v d u d n \\
\quad=\int_{V_{M}^{\#} \backslash N^{\#}} \int_{U}\left\{W_{s}\left(\cdot w_{U} v_{1}\right), W_{-s}^{\vee}\left(\cdot w_{U} v_{2}\right)\right\} \psi_{U}\left(v_{1}\right)^{-1} \psi_{N^{\#}}\left(v_{2}\right) d v_{1} d v_{2} .
\end{gathered}
$$

By (5.4), for any $\pi \in \operatorname{Irr}_{\text {gen }} M, W \in \operatorname{Ind}\left(\mathbb{W}^{\psi_{N_{M}}}(\pi)\right)_{\#}^{\circ}, W^{\vee} \in \operatorname{Ind}\left(\mathbb{W}^{\psi_{N_{M}}^{-1}}(\mathfrak{c}(\pi))\right)$ and $-\operatorname{Re} s \gg 1$ we have

$$
\underline{B}\left(W, W^{\vee}, s\right)=B\left(W, W^{\vee}, s\right) .
$$

The following proposition is proved in a similar way as the argument in [32, Appendix B], practically word for word. Hence, we omit its proof.

Proposition 6.1. Let $\pi \in \operatorname{Irr}_{\text {temp }} M$. Then

(1) For $\operatorname{Re} s \gg 1, \underline{B}\left(W, W^{\vee}, s\right)$ is well-defined for any $W \in \operatorname{Ind}\left(\mathbb{W}^{\psi_{N_{M}}}(\pi)\right) W^{\vee} \in \operatorname{Ind}\left(\mathbb{W}^{\psi_{N_{M}}^{-1}}\left(\pi^{\vee}\right)\right)^{\circ}$

(2) For $-\operatorname{Re} s \gg 1, \underline{B}\left(W, W^{\vee}, s\right)$ is well-defined for any $W \in \operatorname{Ind}\left(\mathbb{W}^{\psi_{N_{M}}}(\pi)\right)^{\circ} W^{\vee} \in \operatorname{Ind}\left(\mathbb{W}^{\psi_{N_{M}}}\left(\pi^{\vee}\right)\right)$

(3) For $-\operatorname{Re} s \gg 1$, we have

$$
\begin{aligned}
\underline{B} & \left(W, M(s) W^{\wedge}, s\right)=\underline{B}\left(M(s) W, W^{\wedge},-s\right) \\
\text { for any } W \in \operatorname{Ind}\left(\mathbb{W}^{\psi_{N_{M}}}(\pi)\right)^{\circ} W^{\wedge} & \in \operatorname{Ind}\left(\mathbb{W}^{\psi_{N_{M}}^{-1}}(\pi)\right)^{\circ} .
\end{aligned}
$$

Recall the definition of the space $W^{\vee} \in \operatorname{Ind}\left(\mathbb{W}^{\psi_{N_{M}}^{-1}}(\pi)\right)^{\circ}$ in Section 4.1 .

Combined with the above we get

Corollary 6.1. For $-\operatorname{Re} s \gg 1$ we have

$$
\underline{B}\left(W, M(s) W^{\wedge}, s\right)=\underline{B}\left(M(s) W, W^{\wedge},-s\right)
$$

for any $W \in \operatorname{Ind}\left(\mathbb{W}^{\psi_{N_{M}}}(\pi)\right)_{\#}^{\circ} W^{\wedge} \in \operatorname{Ind}\left(\mathbb{W}^{\psi_{N_{M}}^{-1}}(\pi)\right)^{\circ}$. 
6.1. Put $\varepsilon_{2}=\ell_{\mathbb{M}}\left(e_{1,1}+J\right)$ where $e_{1,1}$ is the matrix in Mat $_{n}$ with 1 in the upper left corner and zero elsewhere $\varepsilon_{3}=w_{2 n, n}^{\prime} \varepsilon_{2}$ and $\varepsilon_{4}$ an arbitrary element of $N_{\mathbb{M}}$. As in [44, Section 9], define

$$
\Delta(t):=\left|t_{1}\right|^{-n} \delta_{B}^{\frac{1}{2}}(\varrho(t)), \quad t=\operatorname{diag}\left(t_{1}, \ldots, t_{2 n}\right) \in T_{\mathbb{M}} .
$$

In particular, when $t \in \eta_{\mathbb{M}}^{\vee}\left(t^{\prime}\right)$ with $t^{\prime} \in T_{\mathbb{M}^{\prime}}^{\prime}$, we have $\Delta(t)=\delta_{B^{\prime}}^{\frac{1}{2}}\left(\varrho^{\prime}\left(t^{\prime}\right)\right)$.

Let $T^{\prime \prime}=\eta_{\mathbb{M}^{\prime}}^{\vee}\left(T_{\mathbb{M}^{\prime}}^{\prime}\right) \times Z_{\mathbb{M}}$. For any $W \in C^{\mathrm{sm}}\left(N \backslash G, \psi_{N}\right)$ and $t \in T^{\prime \prime}$, define

$$
\begin{aligned}
E^{\psi}(W, t) & :=\Delta(t)^{-1} \int_{\eta_{M}\left(N_{\mathbb{M}^{\prime}}^{\prime}\right) \backslash N^{\#}} W\left(\varrho\left(t \varepsilon_{4} \varepsilon_{3}\right) w_{U} v\right) \psi_{N \#}(v)^{-1} d v \\
& =\Delta(t)^{-1} \psi_{N_{\mathbb{M}}}\left(t \varepsilon_{4} t^{-1}\right) \int_{\eta_{M}\left(N_{\mathbb{M}^{\prime}}^{\prime}\right) \backslash N^{\#}} W\left(\varrho\left(t \varepsilon_{3}\right) w_{U} v\right) \psi_{N \#}(v)^{-1} d v
\end{aligned}
$$

Definition 6.1. Recall the definition of a certain subspace of $\mathbb{W}^{\psi_{N_{\mathbb{M}}}^{-1}}(\pi)$ in [30, Definition 7.5]. Let $\mathbb{W}^{\psi_{N_{\mathbb{M}}}^{-1}}(\pi)_{\natural}$ be the subspace of $\mathbb{W}^{\psi_{N_{\mathbb{M}}}^{-1}}(\pi)$ consisting of $W$ such that

$$
\left.W\left(\cdot \varepsilon_{3}\right)\right|_{\mathcal{P}^{*}} \in C_{c}^{\infty}\left(N_{\mathbb{M}} \backslash \mathcal{P}^{*}, \psi_{N_{\mathbb{M}}}^{-1}\right) \text { and }\left.W\left(\cdot \varepsilon_{3}\right)\right|_{\eta_{\mathbb{M}^{\prime}}\left(T_{\mathbb{M}^{\prime}}^{\prime}\right) \ltimes \bar{R}} \in C_{c}^{\infty}\left(\eta_{\mathbb{M}^{\vee}}^{\vee}\left(T_{\mathbb{M}^{\prime}}^{\prime}\right) \ltimes \bar{R}\right) \text {. }
$$

As remarked in [30, p.33], this space is non-zero, and thus the following space is also non-zero.

Definition 6.2. Let $\operatorname{Ind}\left(\mathbb{W}^{\psi_{N_{M}}^{-1}}(\pi)\right)_{\square}^{\circ}$ be the linear subspace of $\operatorname{Ind}\left(\mathbb{W}_{N_{M}}^{\psi_{M}^{-1}}(\pi)\right)^{\circ}$ spanned by the functions which vanish outside $P w_{U} N$ and on the big cell are given by

$$
W\left(u^{\prime} m w_{U} u\right)=\delta_{P}^{\frac{1}{2}}(m) W^{M}(m) \phi(u), \quad m \in M, u, u^{\prime} \in U
$$

with $\phi \in C_{c}^{\infty}(U)$ and $W^{M} \circ \varrho \in \mathbb{W}^{\psi_{N_{M}}^{-1}}(\pi)_{\text {七. }}$.

In the same argument as the proof of [30, Lemma 7.9], the following lemma is proved.

Lemma 6.1. Let $\pi \in \operatorname{Irr}_{\text {gen }} M$. For $\operatorname{Re} s \gg 1$ the integral (7.1) defining $E^{\psi}\left(W_{s}, t\right)$ converges for any $W \in \operatorname{Ind}\left(\mathbb{W} \psi_{N_{M}}(\pi)\right)$ and $t \in S$ uniformly for $(s, t)$ in a compact set. Hence, $E^{\psi}\left(W_{s}, t\right)$ is holomorphic for $\operatorname{Re} s \gg 1$ and continuous in t. If $W^{\wedge} \in \operatorname{Ind}\left(\mathbb{W}^{\psi_{N_{M}}^{-1}}(\pi)\right)^{\circ}$ then $E^{\psi^{-1}}\left(W_{s}^{\wedge}, t\right)$ is entire in $s$ and locally constant in $t$, uniformly in s. If moreover $W^{\wedge} \in \operatorname{Ind}\left(\mathbb{W}^{\psi_{N_{M}}^{-1}}(\pi)\right)_{\natural}^{\circ}$ then $E^{\psi^{-1}}\left(W_{s}^{\wedge}, t\right)$ is compactly supported in $t \in S$, uniformly in s.

Proposition 6.2. Let $\pi \in \operatorname{Irr}_{\text {temp }} M$. Then for $\operatorname{Re} s \gg 1$ we have

$$
\underline{B}\left(W, W^{\vee}, s\right)=\int_{\left.\eta_{\mathbb{M}^{\vee}\left(T_{\mathbb{M}^{\prime}}^{\prime}\right.}\right)} E^{\psi}\left(W_{s}, t\right) E^{\psi^{-1}}\left(W_{-s}^{\vee}, t\right) \frac{d t}{|\operatorname{det} t|}
$$

for any $W \in \operatorname{Ind}\left(\mathbb{W}^{\psi_{N_{M}}}(\pi)\right) W^{\vee} \in \operatorname{Ind}\left(\mathbb{W}^{\psi_{N_{M}}^{-1}}(\pi)\right)_{\natural}^{\circ}$ where the integrand on the right-hand side is continuous and compactly supported.

Proof. This is proved in the same argument as the proof of [30, Proposition 7.10] using (6.1) and Lemma 6.1] instead of (7.1) and Lemma 7.9 in [30, respectively.

Combining Proposition 6.2 with Corollary 6.1 we get

Proposition 6.3. Let $\pi \in \operatorname{Irr}_{\text {temp }} M$. Then for $-\operatorname{Re} s \gg 1$ and anyW $\in \operatorname{Ind}\left(\mathbb{W}^{\psi_{N_{M}}}(\pi)\right)_{\#}^{\circ} W^{\vee} \in \operatorname{Ind}\left(\mathbb{W}^{\psi_{N_{M}}^{-1}}(\pi)\right)_{\natural}^{\circ}$, we have

$$
B\left(W, M(s) W^{\vee}, s\right)=\int_{S} E^{\psi}\left(M_{s}^{*} W, t\right) E^{\psi^{-1}}\left(W_{s}^{\vee}, t\right) \frac{d t}{|\operatorname{det} t|}
$$

where the integrand is continuous and compactly supported. Here, for the simplicity, we denote $S=\eta_{\mathbb{M}^{\mathbb{V}}}^{\vee}\left(T_{\mathbb{M}^{\prime}}^{\prime}\right)$.

\section{Proof of Proposition 5.1}

7.1. Let $\mathfrak{d}=\operatorname{diag}\left(1,-1, \ldots,(-1)^{n-1}\right) \in$ Mat $_{n}$. We now fix

$$
\varepsilon_{4}=\ell_{\mathbb{M}}\left(-\frac{1}{2} \mathfrak{d} w_{0}^{\mathbb{M}^{\prime}}\right) \in N_{\mathbb{M}}
$$

This element is denoted by $\varepsilon^{\prime}$ in the beginning of [44, Section 8] with the parameter $\mathfrak{a}=-\frac{1}{2}$ in the notation of that paper. We also fix $\varepsilon_{2}=\ell_{\mathbb{M}}(\mathfrak{d})$ (and correspondingly $\varepsilon_{3}=w_{2 n, n}^{\prime} \varepsilon_{2}$ ). Then we have

$$
\psi_{\bar{U}}(\bar{v})=\psi_{U}\left(\left(\varrho\left(\varepsilon_{4} \varepsilon_{3}\right) w_{U}\right)^{-1} \bar{v} \varrho\left(\varepsilon_{4} \varepsilon_{3}\right) w_{U}\right)^{-1}=\psi\left(\bar{v}_{2 n+1,1}\right), \quad \bar{v} \in \bar{U} .
$$


We note that in [44, 4.1], the character $\psi_{\bar{U}}$ is denoted by $\psi_{\bar{U}, F}$. As in [30, 8.1], we may rewrite (for $\left.\operatorname{Re} s \gg 1\right)$

$$
\begin{aligned}
E^{\psi}\left(W_{s}, t\right)=\Delta(t)^{-1} \int_{\eta_{\mathbb{M}}^{\vee}\left(N_{\mathbb{M}^{\prime}}^{\prime}\right) \backslash N_{\mathbb{M}}^{\mathrm{b}}} \int_{\bar{U}} W_{s}\left(\varrho(t) \bar{v} \varrho\left(\varepsilon_{4} \varepsilon_{3} r\right) w_{U}\right) \psi_{\bar{U}}(\bar{v}) \psi_{N_{\mathbb{M}}^{\mathrm{b}}}(r)^{-1} d \bar{v} d r \\
=\Delta(t)^{-1} \int_{\bar{R}} \int_{\bar{U}} W_{s}\left(\varrho(t) \bar{v} \varrho\left(\varepsilon_{4} r \varepsilon_{3}\right) w_{U}\right) \psi_{\bar{U}}(\bar{v}) \psi_{\bar{R}}(r) d \bar{v} d r
\end{aligned}
$$

Here, recall $N_{\mathbb{M}}^{b}=\left(N_{\mathbb{M}}^{\#}\right)^{*}$ and $\psi_{N_{\mathbb{M}}^{b}}(m)=\psi_{N_{\mathbb{M}}^{\#}}\left(m^{*}\right)$, and define $\psi_{\bar{R}}(r)=\psi_{N_{\mathbb{M}}^{b}}\left(\varepsilon_{3}^{-1} r \varepsilon_{3}\right)^{-1}$, and

$$
\bar{R}=\varepsilon_{3}\left(\eta_{\mathbb{M}^{\mathbb{M}}}\left(N_{\mathbb{M}^{\prime}}^{\prime}\right) \ltimes \ell_{\mathbb{M}}(J)\right) \varepsilon_{3}^{-1}=w_{2 n, n}^{\prime}\left(\eta_{\mathbb{M}^{\mathbb{M}}}\left(N_{\mathbb{M}^{\prime}}^{\prime}\right) \ltimes \ell_{\mathbb{M}}(J)\right) w_{2 n, n}^{\prime-1}=\left\{\left(\begin{array}{cc}
I_{n} & \\
x & { } n
\end{array}\right): x \in J, n \in N_{\mathbb{M}^{\prime}}^{\prime}\right\} \subset{ }^{t} N_{\mathbb{M}} \cap \mathcal{P}^{*} .
$$

We now quote the following pertinent result from [4]. Let

$$
T_{i}:=\left\{\operatorname{diag}\left(1_{i-1}, z, 1_{2 n-i}\right): z \in E^{\times}\right\}
$$

Then we have $S=\prod_{i=1}^{n} T_{i}$. For any $f \in C_{c}^{\infty}(S)$ and $g \in C(S)$, we write $f * g(\cdot)=\int_{S} f(t) g(\cdot t) d t$.

Theorem 7.1. Let $K_{i}$ be a compact open subgroup of $E^{\times}$and $f_{K_{i}}$ be the characteristic function of $K_{i}$. Regard $f_{K_{i}}$ as a function on $T_{i}$, and put $f=f_{K_{1}} \otimes \cdots \otimes f_{K_{n}} \in \otimes_{i} C_{c}^{\infty}\left(T_{i}\right)=C_{c}^{\infty}(S)$.

For any $W \in C^{\operatorname{sm}}\left(N \backslash G, \psi_{N}\right)$ which is left invariant under a compact open subgroup of $Z_{M}$, the function $f * E^{\psi}\left(W_{s}, t\right)$ extends to an entire function in $s$ which is locally constant in $t$, uniformly in s. Moreover, if $\pi \in \operatorname{Irr}_{\mathrm{ut}, \text { temp }} \mathbb{M}$ then

$$
\left.f * E^{\psi}\left(W_{s}, t\right)\right|_{s=\frac{1}{2}}= \begin{cases}\omega_{\pi}(\tau)^{n} A_{e}^{\psi}\left(M^{*} W\right) \int_{S_{F}} f\left(t^{\prime}\right) d t^{\prime} & \text { if } t_{i} \in F^{\times} K_{i} \\ 0 & \text { otherwise }\end{cases}
$$

for $t=\operatorname{diag}\left(1, \ldots 1, t_{1}, \ldots, t_{n}\right)$. Here, $S_{F}=S \cap \mathrm{GL}_{4 n}(F)$. by

Let us explain that this theorem follows from result in [44. As in [44, 4.1], let $\mathcal{Z}$ be the unipotent subgroup of $\mathbb{M}$ given

$$
\mathcal{Z}=\left\{m \in \mathbb{M}: m_{i, i}=1 \forall i, m_{i, j}=0 \text { if either }(j>i \text { and } i+j>2 n) \text { or }(i>j \text { and } i+j \leq 2 n+1)\right\}
$$

and let $\psi_{\mathcal{Z}, F}$ be its character

$$
\psi_{\mathcal{Z}, F}(m)=\psi\left(m_{1,2}+\cdots+m_{n-1, n}-m_{n+2}-\cdots-m_{2 n, 2 n-1}\right) .
$$

The group $\varrho(\mathcal{Z})$ stabilizes the character $\psi_{\bar{U}}$. Let $\mathfrak{E}=\varrho(\mathcal{Z}) \ltimes \bar{U}$. Also. let $\mathcal{Z}^{+}=\mathcal{Z} \cap N_{\mathbb{M}}, V_{\Delta}=\mathcal{Z} \cap{ }^{t} N_{\mathbb{M}}$ and

$$
N_{\mathbb{M}, \Delta}=\left\{{ }^{t} \ell_{\mathbb{M}}(X):{ }^{t} X \in J, X_{i, j}=0 \text { if } i+j>n+1\right\} .
$$

We have $\mathcal{Z}=\mathcal{Z}^{+} \cdot V_{\Delta}$ and $\bar{R}=V_{\Delta} \cdot N_{\mathbb{M}, \Delta}$.

The character $\psi_{\bar{R}}$ is given by $\psi_{\bar{R}}(v n)=\psi_{V_{\Delta}, F}(v), v \in V_{\Delta}, n \in N_{\mathbb{M}, \Delta}$ where $\psi_{v_{\Delta}, F}(v)$ is defined in [44, 4.1]. Then the expression in [44, (10.6)] evaluated at $\varrho\left(\varepsilon_{3}\right) w_{U}$ is $(f \Delta) * E^{\psi}\left(W_{s}, t\right)$, and its analytic continuation is given by the expression in [44, (10.5)] which amounts to a finite sum.

Meanwhile, from the definition of $A_{e}^{\psi}$ in (5.1) we have

$$
A_{e}^{\psi}(W)=\int_{\varrho\left(V_{\mathbb{M}}^{*}\right) \backslash \gamma V_{-} \gamma^{-1}} W\left(v \gamma \varepsilon_{1}\right) \psi_{V_{-}}\left(\left(\gamma \varepsilon_{1}\right) v \gamma \varepsilon_{1}\right)^{-1} d v .
$$

We can integrate over the group $\bar{U}\urcorner$ in [44, (10.2)], consisting of elements $\bar{u} \in \bar{U}$ such that $\bar{u}_{2 n+i, j}=$ whenever $i \geq n$ and $j \leq n$. Then the character $\bar{u} \mapsto \psi_{V_{-}}\left(\left(\gamma \varepsilon_{1}\right)^{-1} \bar{u} \gamma \varepsilon_{1}\right)$ on $\bar{U}^{\urcorner}$is the character denoted by $\hat{\psi}_{\bar{U}^{\Delta}}$ in the definition of $\mathcal{T}^{\prime}$ in [44, 10.2]. Thus, $A_{e}^{\psi}(W)$ is $\mathcal{T}^{\prime}(W)\left(\gamma \varepsilon_{1}\right)$ in the notation of [44, 10.2]. The second part amounts to the first statement of [44 Corollary 10.8] upon taking

$$
\varepsilon_{1}=(\hat{w} \gamma)^{-1} \varrho\left(\varepsilon_{3}\right) w_{U}=\ell_{M}\left((-1)^{n} \mathfrak{d}\right)
$$

where

$$
\hat{w}:=\eta\left(w_{U^{\prime}}^{\prime}\right) \varrho\left(w_{2 n, n}^{\prime}\right)=\left(\begin{array}{cccc} 
& I_{n} & & \\
& & & w_{n} \\
-w_{n} & & &
\end{array}\right)
$$

is as in [44, 4.1]. This theorem is crucial step, which is a unitary analogue of [30, Theorem 8.1] and proved in [34. As in [34, the main input is that the Langlands quotient of $\operatorname{Ind}\left(\mathbb{W}^{\psi_{N_{M}}}(\pi), \frac{1}{2}\right)$ admits a realization in $C^{\mathrm{sm}}(H \backslash G)$ where $H=G_{2 n} \cap \mathrm{GL}_{4 n}(F)=\operatorname{Sp}_{4 n}(F)$. We refer to 44 for more details. 
Corollary 7.1. Suppose that $\pi \in \operatorname{Irr}_{\mathrm{ut}, \text { temp }} \mathbb{M}$. Then for any $W \in \operatorname{Ind}\left(\mathbb{W}^{\psi_{N_{M}}}(\pi)\right)_{\#}^{\circ}, W^{\wedge} \in \operatorname{Ind}\left(\mathbb{W}^{\psi_{N}}-1\right.$

$$
B\left(W, M\left(\frac{1}{2}\right) W^{\wedge}, \frac{1}{2}\right)=\omega_{\pi}(\tau)^{n} A_{e}^{\psi}\left(M^{*} W\right) \int_{S_{F}} E^{\psi^{-1}}\left(W_{\frac{1}{2}}^{\wedge}, t\right) \frac{d t}{|\operatorname{det} t|}
$$

where recall $S_{F}=S \cap \mathrm{GL}_{4 n}(F)$.

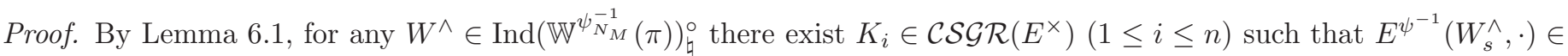
$C(S)^{K_{0}}$ for all $s$ and $E^{\psi^{-1}}\left(W_{s}^{\wedge}, \cdot\right)$ is compactly supported on $S$ uniformly in $s$. Here, we regard $K_{i}$ as a subgroup of $S$ and we put $K_{0}=\prod_{i=1}^{n} K_{i}$. Suppose $f:=f_{K_{1}} \otimes \cdots f_{K_{n}} \in C_{c}^{\infty}(S)$ and let $f^{\vee}(t):=f\left(t^{-1}\right)$. By Proposition 6.3 for - Re $s \gg 1$ and $W \in \operatorname{Ind}\left(\mathbb{W}^{\psi_{N}}{ }_{M}(\pi)\right)_{\#}^{\circ}$ we have

$$
B\left(W, M(s) W^{\wedge}, s\right) \int_{S} f(t) d t=\int_{S} E^{\psi}\left(M_{s}^{*} W, t\right) f^{\vee} * E^{\psi^{-1}}\left(W_{s}^{\wedge}, t\right) \frac{d t}{|\operatorname{det} t|}=\int_{S} f * E^{\psi}\left(M_{s}^{*} W, t\right) E^{\psi^{-1}}\left(W_{s}^{\wedge}, t\right) \frac{d t}{|\operatorname{det} t|}
$$

Since $B\left(W, W^{\wedge}, s\right)$ is entire function of $s$ for $W \in \operatorname{Ind}\left(\mathbb{W}_{N_{M}}(\pi)\right)_{\#}^{\circ}, W^{\vee} \in \operatorname{Ind}\left(\mathbb{W}^{\psi_{N_{M}}^{-1}}(\pi)\right)$, the first part of Theorem 7.1 implies that both sides of (17.3) are meromorphic functions and the identity holds whenever $M(s)$ is holomorphic. Then by [29, Proposition 2.1], we may specialize $s=\frac{1}{2}$. Using the second part of Theorem 7.1] we find that the right-hand side of (7.3) is equal to

$$
\begin{aligned}
\omega_{\pi}(\tau)^{n} A_{e}^{\psi}\left(M^{*} W\right)\left(\int_{S_{F}} f(t) d t\right) & \int_{S \cap K_{0}^{\prime}} E^{\psi^{-1}}\left(W_{\frac{1}{2}}^{\wedge}, t\right) \frac{d t}{|\operatorname{det} t|} \\
& =\omega_{\pi}(\tau)^{n} A_{e}^{\psi}\left(M^{*} W\right)\left(\int_{S_{F}} f(t) d t\right) \cdot\left(\int_{K_{0} \cap \mathrm{GL}_{4 n}(F) \backslash K_{0}} d t\right) \int_{S_{F}} E^{\psi^{-1}}\left(W_{\frac{1}{2}}^{\wedge}, t\right) \frac{d t}{|\operatorname{det} t|}
\end{aligned}
$$

where $K_{0}^{\prime}=\prod_{i=1}^{n}\left(F^{\times} K_{i}\right)$. The required formula readily follows since

$$
\left(\int_{S_{F}} f(t) d t\right) \cdot\left(\int_{K_{0} \cap \mathrm{GL}_{4 n}(F) \backslash K_{0}} d t\right)=\int_{S} f(t) d t
$$

7.2. It remains to compute the integral on the right-hand side of (7.2). Again this is essentially done in 44 and it relies heavily on the fact that $\pi \in \operatorname{Irr}_{\mathrm{ut}} \mathbb{M}$.

Let

$$
\mathbb{W}^{\psi_{N_{\mathbb{M}}}}(\pi)_{\text {明 }}=\left\{W \in \mathbb{W}^{\psi_{N_{\mathbb{M}}}}(\pi):\left.W\right|_{\mathcal{P}^{*}} \in C_{c}^{\infty}\left(N_{\mathbb{M}} \backslash \mathcal{P}^{*}, \psi_{N_{\mathbb{M}}}\right) \text { and }\left.W\right|_{\eta_{\mathbb{M}}^{\vee}\left(T_{\mathbb{M}^{\prime}}^{\prime}\right) \ltimes \mathcal{Z}} \in C_{c}^{\infty}\left(\mathcal{Z}^{+} \backslash \eta_{\mathbb{M}^{\prime}}^{\vee}\left(T_{\mathbb{M}^{\prime}}^{\prime}\right) \ltimes \mathcal{Z}, \psi_{\mathcal{Z}}\right)\right. \text {. }
$$

Theorem 7.2. Let $\pi \in \operatorname{Irr}_{\mathrm{ut}, \text { temp }} \mathbb{M}$. Then

(1) (see [46, Corollary 4.1] and [44, Lemma 3.5].) The integral

$$
\mathfrak{P}^{H_{\mathbb{M}}}(W):=\int_{\left(H_{\mathbb{M} \cap} \cap N_{\mathbb{M}}\right) \backslash H_{\mathbb{M}} \cap \mathcal{P}} W\left(\tau^{*} p\right) d p
$$

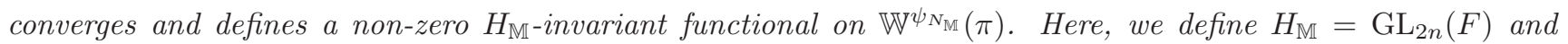
$\tau^{*}=\operatorname{diag}(\tau, 1, \tau, 1, \ldots, \tau, 1) \in \mathbb{M}$.

(2) (44, Corollary 10.4]) Put

$$
x(n)= \begin{cases}n & \text { if } n \text { is even, } \\ 0 & \text { if } n \text { is odd }\end{cases}
$$

which is $b^{\prime \prime}(n)$ in the notation of [44]. Then for any $W \in \mathbb{W}^{\psi_{N_{\mathbb{M}}}}(\pi)_{\text {吅 }}$ we have

$$
\int_{\mathcal{Z}+\backslash \mathcal{Z}} \int_{S_{F}} \Delta(t)^{-1}|\operatorname{det} t|^{n-\frac{1}{2}} W(t r) \psi_{\mathcal{Z}, F}(r)^{-1} d t d r=\omega_{\pi}(\tau)^{n}|\tau|^{x(n)} \int_{\mathcal{Z} \cap H_{\mathbb{M} \backslash \mathcal{Z}}} \mathfrak{P}^{H_{\mathbb{M}}}\left(\pi\left(n \tau_{\circ}\right) W\right) \psi_{\mathcal{Z}}(n)^{-1} d n .
$$

where $\tau_{\circ} \in \mathbb{M}$ is defined by $\varrho\left(\tau_{\circ}\right)=\hat{w} \varrho\left(\tau^{*}\right)^{-1} \hat{w}^{-1}$ and we define $\psi_{\mathcal{Z}}(m)=\psi_{\mathcal{Z}, F}\left(\tau_{\circ}^{-1} m \tau_{\circ}\right)$.

(3) (44, Lemma 4.2]) The integral

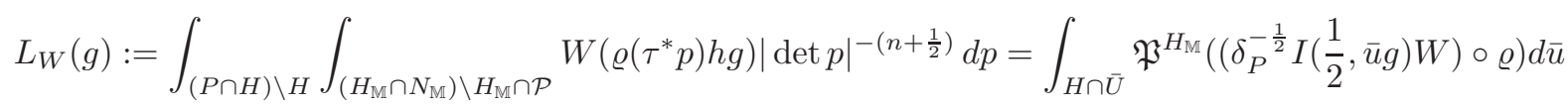

converges for any $W \in \operatorname{Ind}\left(\mathbb{W}^{\psi_{N_{M}}}(\pi), \frac{1}{2}\right)$ and defines an intertwining map

$$
\operatorname{Ind}\left(\mathbb{W}^{\psi_{N_{M}}}(\pi), \frac{1}{2}\right) \rightarrow C^{\mathrm{sm}}(H \backslash G) .
$$


(4) (second statement of [44, Corollary 10.8]) Put

$$
y(n)= \begin{cases}n & \text { if } n \text { is even, } \\ n-\frac{1}{2} & \text { if } n \text { is odd }\end{cases}
$$

which is $a(n)+n^{2}$ in the notation of [44]. Then we have

$$
A_{e}^{\psi}\left(M^{*} W\right)=\omega_{\pi}(\tau)|\tau|^{y(n)}\left(\int_{N_{\mathbb{M}, \Delta}} \int_{H \cap \mathfrak{E} \backslash \mathfrak{E}} L_{W}\left(v \varrho\left(\tau_{\circ} \varepsilon_{4} u \varepsilon_{3}\right) w_{U}\right) \psi_{\mathfrak{E}}^{-1}(v) d v\right) d u
$$

where we define

$$
\psi_{\mathfrak{E}}(\varrho(m) \bar{u})=\psi_{\mathcal{Z}, F}\left(\tau_{\circ}^{-1} m \tau_{\circ}\right) \psi_{\bar{U}}^{-1}\left(\tau_{\circ}^{-1} \bar{u} \tau_{\circ}\right)=\psi_{\mathcal{Z}}(m) \psi_{\bar{U}}^{-1}\left(\tau_{\circ}^{-1} \bar{u} \tau_{\circ}\right) .
$$

Corollary 7.2. Let $\pi \in \operatorname{Irr}_{\mathrm{ut}, \text { temp }} \mathbb{M}$. Then for any $W \in \operatorname{Ind}\left(\mathbb{W} \psi_{N_{M}}(\pi)\right)_{\mathrm{b}}^{\circ}$ we have

$$
\int_{S_{F}} E^{\psi}\left(W_{\frac{1}{2}}, t\right) \frac{d t}{|\operatorname{det} t|}=\omega_{\pi}(\tau)^{n+1} A_{e}^{\psi}\left(M^{*} W\right) .
$$

Proof. We may assume without loss of generality that

$$
W_{\frac{1}{2}}\left(u^{\prime} \varrho(m) w_{U} u\right)=W^{\mathbb{M}}(m)|\operatorname{det} m|^{\frac{1}{2}} \delta_{P}(\varrho(m))^{\frac{1}{2}} \phi(u), \quad m \in \mathbb{M}, u, u^{\prime} \in U
$$

with $W^{\mathbb{M}} \in \mathbb{W}^{\psi_{N_{M}}}(\pi)_{\natural}$ and $\phi \in C_{c}^{\infty}(U)$. We evaluate the left-hand side $I$ of (7.5) using the last expression in (7.1) (where we recall that the integrand is compactly supported by Lemma 6.1). Thus,

$$
I=I^{\prime} \int_{U} \phi(u) \psi_{U}^{-1}(v) d v
$$

where

$$
I^{\prime}=\int_{S_{F}} \int_{\bar{R}} \Delta(t)^{-1}|\operatorname{det} t|^{n-\frac{1}{2}} W^{\mathbb{M}}\left(t \varepsilon_{4} r \varepsilon_{3}\right) \psi_{\bar{R}}(r) d r d t
$$

The integrand in $I^{\prime}$ is compactly supported because $W^{\mathbb{M}} \in \mathbb{W}^{\psi_{N_{\mathbb{M}}}}(\pi)_{\text {घ. }}$. Note for $r \in V_{\Delta}=\mathcal{Z} \cap \bar{R}, \psi_{\bar{R}}(r)^{-1}=\psi_{\mathcal{Z}, F}(r)$. We write

$$
\begin{aligned}
I^{\prime}=\int_{N_{\mathbb{M}, \Delta}}\left(\int_{V_{\Delta}} \int_{S_{F}} \Delta(t)^{-1}|\operatorname{det} t|^{n-\frac{1}{2}} W^{\mathbb{M}}\left(t \varepsilon_{4} r u \varepsilon_{3}\right) \psi_{\mathcal{Z}, F}(r)^{-1} d t d r\right) d u \\
=\int_{N_{\mathbb{M}, \Delta}}\left(\int_{\mathcal{Z}+\backslash \mathcal{Z}} \int_{S_{F}} \Delta(t)^{-1}|\operatorname{det} t|^{n-\frac{1}{2}} W^{\mathbb{M}}\left(t \varepsilon_{4} r u \varepsilon_{3}\right) \psi_{\mathcal{Z}, F}(r)^{-1} d t d r\right) d u \\
\quad=\int_{N_{\mathbb{M}, \Delta}}\left(\int_{\mathcal{Z}+\backslash \mathcal{Z}} \int_{S_{F}} \Delta(t)^{-1}|\operatorname{det} t|^{n-\frac{1}{2}} W^{\mathbb{M}}\left(\operatorname{tr} \varepsilon_{4} u \varepsilon_{3}\right) \psi_{\mathcal{Z}, F}(r)^{-1} d t d r\right) d u
\end{aligned}
$$

since $\varepsilon_{4}$ stabilizes $\psi_{\mathcal{Z}, F}$. For the double integral in the brackets we apply part 2 of the theorem above to $\pi\left(\varepsilon_{4} u \varepsilon_{3}\right) W^{\mathbb{M}}$ (which is applicable since $W^{\mathbb{M}} \in \mathbb{W}^{\psi_{N_{\mathbb{M}}}}(\pi)_{\natural}$ ). We get

$$
I^{\prime}=\omega_{\pi}(\tau)^{n}|\tau|^{x(n)} \int_{N_{\mathbb{M}, \Delta}}\left(\int_{\mathcal{Z} \cap H_{\mathbb{M}} \backslash \mathcal{Z}} \mathfrak{P}^{H_{\mathbb{M}}}\left(\pi\left(n \tau_{\circ} \varepsilon_{4} u \varepsilon_{3}\right) W^{\mathbb{M}}\right) \psi_{\mathcal{Z}}(n)^{-1} d n\right) d u
$$

Thus,

$$
I=\omega_{\pi}(\tau)^{n}|\tau|^{x(n)} \int_{N_{\mathbb{M}, \Delta}}\left(\int_{\mathcal{Z} \cap H_{\mathbb{M}} \backslash \mathcal{Z}} \int_{U} \mathfrak{P}^{H_{\mathbb{M}}}\left(\pi\left(n \tau_{\circ} \varepsilon_{4} u \varepsilon_{3}\right) W^{\mathbb{M}}\right) \psi_{\mathcal{Z}}(n)^{-1} \phi(v) \psi_{U}(v)^{-1} d v d n\right) d u
$$

From (7.6),

$$
\left(\delta_{P}^{-\frac{1}{2}} I\left(\frac{1}{2}, \varrho(m) w_{U} v\right) W\right) \circ \varrho=\phi(v) \delta_{P}^{\frac{1}{2}}(\varrho(m))|\operatorname{det} m|^{\frac{1}{2}} \pi(m) W^{\mathbb{M}}
$$

for any $v \in U$ and $m \in \mathbb{M}$. Thus, $I$ equals

$$
\omega_{\pi}(\tau)^{n}|\tau|^{x(n)} \delta_{P}^{-\frac{1}{2}}\left(\varrho\left(\tau_{\circ}\right)\right)\left|\operatorname{det} \tau_{\circ}\right|^{-\frac{1}{2}} \int_{N_{\mathbb{M}, \Delta}}\left(\int_{\mathcal{Z} \cap H_{\mathbb{M}} \backslash \mathcal{Z}} \int_{U} \mathfrak{P}^{H_{\mathbb{M}}}\left(\delta_{P}^{-\frac{1}{2}} I\left(\pi, \varrho\left(n \tau_{\circ} \varepsilon_{4} u \varepsilon_{3}\right) w_{U} v\right) W\right) \psi_{\mathcal{Z}}(n)^{-1} \psi_{U}(v)^{-1} d v d n\right) d u
$$

Since $\varepsilon_{3}^{-1} N_{\mathbb{M}, \Delta} \varepsilon_{3} \subset N_{\mathbb{M}}^{b}$, the group $\varrho\left(\varepsilon_{3}^{-1} N_{\mathbb{M}, \Delta} \varepsilon_{3}\right)$ stabilizes the character $\psi_{U}\left(w_{U}^{-1} \cdot w_{U}\right)$ on $\bar{U}$. Making a change of variable

$$
v \mapsto\left(\varrho\left(n \tau_{\circ} \varepsilon_{4} u \varepsilon_{3}\right) w_{U}\right)^{-1} \bar{v} \varrho\left(n \tau_{\circ} \varepsilon_{4} u \varepsilon_{3}\right) w_{U}
$$


on $U$ we obtain

$$
\begin{aligned}
& I=\omega_{\pi}(\tau)^{n}|\tau|^{x(n)} \delta_{P}^{\frac{1}{2}}\left(\varrho\left(\tau_{\circ}\right)\right)\left|\operatorname{det} \tau_{\circ}\right|^{-\frac{1}{2}} \int_{N_{\mathbb{M}, \Delta}}\left(\int_{\varrho\left(\mathcal{Z} \cap H_{\mathbb{M}}\right) \backslash \mathfrak{E}} \mathfrak{P}^{H_{\mathbb{M}}}\left(\delta_{P}^{-\frac{1}{2}} I\left(\pi, v \varrho\left(\tau_{\circ} \varepsilon_{4} u \varepsilon_{3}\right) w_{U}\right) W\right) \psi_{\mathfrak{E}}(n)^{-1} d v d u\right. \\
& \quad=\omega_{\pi}(\tau)^{n}|\tau|^{x(n)} \delta_{P}^{\frac{1}{2}}\left(\varrho\left(\tau_{\circ}\right)\right)\left|\operatorname{det} \tau_{\circ}\right|^{-\frac{1}{2}} \int_{N_{\mathbb{M}, \Delta}}\left(\int_{\mathfrak{E} \cap H) \backslash \mathfrak{E}} \int_{H \cap \bar{U}} \mathfrak{P}^{H_{\mathbb{M}}}\left(\delta_{P}^{-\frac{1}{2}} I\left(\pi, x v \varrho\left(\tau_{\circ} \varepsilon_{4} u \varepsilon_{3}\right) w_{U}\right) W\right) \psi_{\mathfrak{E}}(n)^{-1} d x d v d u\right.
\end{aligned}
$$

From part 3 of the theorem, we get

$$
I=\omega_{\pi}(\tau)^{n}|\tau|^{x(n)} \delta_{P}^{\frac{1}{2}}\left(\varrho\left(\tau_{\circ}\right)\right)\left|\operatorname{det} \tau_{\circ}\right|^{-\frac{1}{2}} \int_{N_{\mathbb{M}, \Delta}}\left(\int_{\mathfrak{E} \cap H) \backslash \mathfrak{E}} L_{W}\left(v \varrho\left(\tau_{\circ} \varepsilon_{4} u \varepsilon_{3}\right) w_{U}\right) \psi_{\mathfrak{E}}(n)^{-1} d x d v d u .\right.
$$

From the last part of the theorem, this is equal to

$$
\omega_{\pi}(\tau)^{n+1}|\tau|^{x(n)-y(n)} \delta_{P}^{\frac{1}{2}}\left(\varrho\left(\tau_{\circ}\right)\right)\left|\operatorname{det} \tau_{\circ}\right|^{-\frac{1}{2}} A_{e}^{\psi}\left(M^{*} W\right)=\omega_{\pi}(\tau)^{n+1} A_{e}^{\psi}\left(M^{*} W\right),
$$

where we used the fact $|\tau|^{x(n)-y(n)} \delta_{P}^{\frac{1}{2}}\left(\varrho\left(\tau_{\circ}\right)\right)\left|\operatorname{det} \tau_{\circ}\right|^{-\frac{1}{2}}=1$ (see [44, Remark 10.1]).

Let us complete a proof of Proposition [5.1. From Corollaries 7.1] and 7.2. we find that (5.5) holds for any $W \in$ $\operatorname{Ind}\left(\mathbb{W}^{\psi_{N_{M}}}(\pi)\right)_{\#}^{\circ}$ and $W^{\wedge} \in \operatorname{Ind}\left(\mathbb{W}^{\psi_{N_{M}}}(\pi)\right)^{\circ}$, namely

$$
B\left(W, M\left(\frac{1}{2}\right) W^{\wedge}, \frac{1}{2}\right)=\omega_{\pi}(\tau) A_{e}^{\psi}\left(M^{*} W\right) A_{e}^{\psi^{-1}}\left(M^{*} W^{\wedge}\right)
$$

On the other hand, as in [30, Section 8C], in a similar argument of the proof of [30, Lemma 6.13] with (7.1), we see that the linear map Ind $\left(\mathbb{W}^{\psi_{N_{M}}}(\pi)\right)_{\natural}^{\circ} \rightarrow C_{c}^{\infty}\left(T_{\mathbb{M}^{\prime}}^{\prime}\right)$ given by $W^{\wedge} \mapsto E^{\psi}\left(W_{\frac{1}{2}}^{\wedge}, \cdot\right)$ is onto. Therefore by Corollary 7.2 the linear form $A_{e}^{\psi^{-1}}\left(M^{*} W^{\wedge}\right)$ does not vanish on $\operatorname{Ind}\left(\mathbb{W}^{\psi_{N_{M}}}(\pi)\right)_{\text {घ. }}^{\circ}$ From Corollary [5.1] we conclude that (5.5) holds for all $W^{\wedge}$, which complete a proof of Proposition 5.1 .

\section{Refined formal Degree conjecture And Conjecture 2}

8.1. Non-archimedean case. In this section, we show an equivalence between a refined formal degree conjecture and Theorem 1.2 for discrete series representations of $G^{\prime}=\mathrm{U}_{2 n}$. In particular, we obtain a proof of refined formal degree conjecture in our case. Suppose that a base field $F$ is a non-archimedean local field of characteristic zero.

Let us recall refined formal degree conjecture by Gross-Reeder [16] and Gan-Ichino [12, 14.5]. For an irreducible discrete series representation of $G^{\prime}$, the formal degree $d_{\sigma}$ for $\sigma$ is the measure on $G^{\prime}$ satisfying

$$
\int_{G^{\prime}}\left(\sigma(g) v_{1}, v_{1}^{\prime}\right)\left(\sigma\left(g^{-1}\right) v_{2}, v_{2}^{\prime}\right) d_{\sigma} g=\left(v_{1}, v_{2}^{\prime}\right)\left(v_{2}, v_{1}^{\prime}\right)
$$

holds for any $v_{1}, v_{1}^{\prime}, v_{2}, v_{2}^{\prime}$ where $(\cdot, \cdot)$ is a $G^{\prime}$-invariant inner product on $V_{\sigma}$. On the other hand, we denote the measure on $G^{\prime}$ defined in Section 2.4 by $d_{\psi}$ in order to clarify the difference of these measures.

Hiraga, Ichino and Ikeda [18 formulated a conjecture on a relationship between these measures $d_{\sigma}$ and $d_{\psi}$ in terms of absolute values of special values of adjoint gamma factors. Recently, Gan-Ichino [12] computed the sign of this special value for Seinberg representation of classical groups and and using an important observation by Gross-Reeder [16 on the sign, Gan-Ichino [12, Section 14.5] conjectured a refinement of [18, Conjecture 1.4]. Indeed, we prove this refined version.

Theorem 8.1. Let $\pi$ be an irreducible representation of $\mathrm{GL}_{2 n}(E)$ of the form $\pi=\tau_{1} \times \cdots \times \tau_{k}$ where $\tau_{i}$ are mutually inequivalent irreducible discrete series representations of $\mathrm{GL}_{n_{i}}(E)$ such that $n=n_{1}+\cdots+n_{k}$ and $L\left(s, \tau_{i}\right.$, As $\left.{ }^{+}\right)$has a pole at $s=0$. Write $\sigma=\mathcal{D}_{\psi^{-1}}^{\Upsilon^{-1}}(\mathfrak{c}(\pi))$, which is an irreducible generic discrete series representation of $G^{\prime}$ (See Theorem A.5). Then the formal degree conjecture

$$
d_{\psi}=2^{k} \lambda(E / F, \psi)^{n} \omega_{\sigma}(-1) \gamma\left(1, \mathfrak{c}(\pi), \mathrm{As}^{-}, \psi\right) d_{\sigma} .
$$

holds for in this case. Here, $\lambda(E / F, \psi)$ is the Langlands' $\lambda$-function and the $\gamma$-factor is defined by Langlands-Shahidi method 49 .

We shall prove this theorem in a very similar argument as the proof of refined formal degree conjecture for metaplectic group by Ichino-Lapid-Mao [20.

Recall the following functional equation. Let $W \in \mathbb{W}^{\psi_{N^{\prime}}^{-1}}(\sigma)$ and $W^{\prime} \in \operatorname{Ind}\left(\mathbb{W}^{\psi_{N_{M}}}(\pi)\right)$. Let $\gamma\left(s, \pi, \mathrm{As}^{ \pm}, \psi\right)$ be Asai gamma factors defined by Langlands-Shahidi method [49]. Then in [45], we proved that

$$
J\left(W, M(s) W^{\prime},-s\right)=\lambda(E / F, \psi)^{n} \frac{\gamma\left(s+\frac{1}{2}, \sigma \times\left(\pi \otimes \Upsilon^{-1}\right), \psi\right)}{\gamma\left(2 s, \mathfrak{c}(\pi), \mathrm{As}^{+}, \psi\right)} J\left(W, W^{\prime}, s\right)
$$


and that the the gamma factor $\gamma\left(s+\frac{1}{2}, \sigma \times\left(\pi \otimes \Upsilon^{-1}\right), \psi\right)$ coincides with the gamma factor defined by Langlands-Shahidi method 49 . We note that the factor $\lambda(E / F, \psi)$ comes from the normalization of an intertwining operator (for example, see Anandavardhanan [1, (3)]).

By Theorem A.5 we have

$$
\gamma\left(s+\frac{1}{2}, \sigma \times\left(\pi \otimes \Upsilon^{-1}\right), \psi\right)=\gamma\left(s+\frac{1}{2}, \pi \otimes \mathfrak{c}(\pi), \psi\right)
$$

Moreover, we have

$$
\gamma\left(s+\frac{1}{2}, \pi \otimes \mathfrak{c}(\pi), \psi\right)=\gamma\left(s+\frac{1}{2}, \mathfrak{c}(\pi), \mathrm{As}^{+}, \psi\right) \gamma\left(s+\frac{1}{2}, \mathfrak{c}(\pi), \mathrm{As}^{-}, \psi\right)
$$

Hence, we get

$$
\lim _{s \rightarrow \frac{1}{2}} \frac{\gamma\left(s+\frac{1}{2}, \sigma \times\left(\pi \otimes \Upsilon^{-1}\right), \psi\right)}{\gamma\left(2 s, \mathfrak{c}(\pi), \mathrm{As}^{+}, \psi\right)}=2^{k} \gamma\left(1, \mathfrak{c}(\pi), \mathrm{As}^{-}, \psi\right)
$$

We define a non-degenerate $G^{\prime}$-invariant bilinear form $(\cdot, \cdot)_{\sigma}$ on $\mathbb{W}^{\psi_{N^{\prime}}^{-1}}(\sigma) \times \mathbb{W}^{\psi_{N^{\prime}}}\left(\sigma^{\vee}\right)$ by

$$
\left(W, W^{\prime}\right)_{\sigma}=\int_{N \backslash G} W(g) W^{\prime}(g) d_{\psi}(g)
$$

which converges absolutely by [10, Proposition 3.2]. On the other hand, recall that we defined a $G^{\prime}$-invariant bilinear form $[\cdot, \cdot]_{\sigma}$ in Definition 3.2 . Indeed, it is defined so that

$$
J\left(W, W^{\prime}, \frac{1}{2}\right)=\left[W, A_{\#}^{\psi, \Upsilon}\left(W^{\prime}, \cdot\right)\right]_{\sigma}
$$

for $W \in \mathbb{W}^{\psi_{N^{\prime}}^{-1}}(\sigma)$ and $W^{\prime} \in \operatorname{Ind}\left(\mathbb{W}^{\psi_{N_{M}}}(\pi)\right)$. Then by the functional equation (8.2), we obtain

$$
\begin{aligned}
\left(W, A_{\#}^{\psi, \Upsilon}\left(M^{*} W^{\prime}, \cdot\right)\right)_{\sigma} & =J\left(W, M^{*} W^{\prime},-\frac{1}{2}\right) \\
& =\lambda(E / F, \psi)^{n} \lim _{s \rightarrow \frac{1}{2}} \frac{\gamma\left(s+\frac{1}{2}, \sigma \times\left(\pi \otimes \Upsilon \Upsilon^{-1}\right), \psi\right)}{\gamma\left(2 s, \mathfrak{c}(\pi), A s^{+}, \psi\right)} J\left(W, W^{\prime}, \frac{1}{2}\right) \\
& =\lambda(E / F, \psi)^{n} \lim _{s \rightarrow \frac{1}{2}} \frac{\gamma\left(s+\frac{1}{2}, \pi \otimes \mathfrak{c}(\pi), \psi\right)}{\gamma\left(2 s, \mathfrak{c}(\pi), \mathrm{As}^{+}, \psi\right)}\left[W, A_{\#}^{\psi, \Upsilon}\left(M^{*} W^{\prime}, \cdot\right)\right]_{\sigma}
\end{aligned}
$$

Hence, by (8.3),

$$
\left(W, A_{\#}^{\psi, \Upsilon}\left(M^{*} W^{\prime}, \cdot\right)\right)_{\sigma}=\lambda(E / F, \psi)^{n} 2^{k} \gamma\left(1, \mathfrak{c}(\pi), \mathrm{As}^{-}, \psi\right)\left[W, A_{\#}^{\psi, \Upsilon}\left(M^{*} W^{\prime}, \cdot\right)\right]_{\sigma}
$$

Recall that the formal degree $d_{\sigma}$ is defined so that

$$
\int_{G^{\prime}}\left[\sigma(g) W_{1}, W_{1}^{\prime}\right]_{\sigma}\left[\sigma\left(g^{-1}\right) W_{2}, W_{2}^{\prime}\right]_{\sigma} d_{\sigma}=\left[W_{1}, W_{2}^{\prime}\right]_{\sigma}\left[W_{2}, W_{1}^{\prime}\right]
$$

for $W_{1}, W_{2} \in \mathbb{W}^{\psi^{\prime}}-1(\sigma)$ and $W_{1}^{\prime}, W_{2}^{\prime} \in \mathbb{W}^{\psi_{N^{\prime}}}(\mathfrak{c}(\sigma))$. Assume that $W_{1}^{\prime}=A_{\#}^{\psi, \Upsilon}\left(M^{*} W^{\prime}, \cdot\right)$. Then by the definition,

$$
\int_{G^{\prime}}\left[\sigma(g) W_{1}, W_{1}^{\prime}\right]_{\sigma}\left[\sigma\left(g^{-1}\right) W_{2}, W_{2}^{\prime}\right] d_{\psi}(g)=\int_{G^{\prime}} \int_{N^{\prime} \backslash G^{\prime}} W_{1}(x g) A_{\#}^{\psi, \Upsilon}\left(M^{*} W^{\prime}, x\right)\left[\sigma\left(g^{-1}\right) W_{2}, W_{2}^{\prime}\right]_{\sigma} d_{\psi}(x) d_{\psi}(g) .
$$

In the same argument as [20, p.1316-1317], we see that this double integral converges absolutely by Lemma 3.3. Then we can change the order of the integration, and changing the variable $g \mapsto x^{-1} g$, we get

$$
\begin{aligned}
\int_{N^{\prime} \backslash G^{\prime}} \int_{G^{\prime}} W_{1}(g) A_{\#}^{\psi, \Upsilon}\left(M^{*} W^{\prime}, x\right)\left[\sigma\left(g^{-1} x\right) W_{2}, W_{2}^{\prime}\right]_{\sigma} d_{\psi}(x) d_{\psi}(g) & \\
=\int_{N^{\prime} \backslash G^{\prime}} \int_{N^{\prime} \backslash G^{\prime}} & \int_{N^{\prime}} \psi_{N^{\prime}}(u)^{-1} W_{1}(g) A_{\#}^{\psi, \Upsilon}\left(M^{*} W^{\prime}, x\right)\left[\sigma\left(g^{-1} u^{-1} x\right) W_{2}, W_{2}^{\prime}\right]_{\sigma} d_{\psi}(x) d_{\psi}(u) d_{\psi}(g) \\
\quad & \int_{N^{\prime} \backslash G^{\prime}} \int_{N^{\prime} \backslash G^{\prime}} \int_{N^{\prime}} \psi_{N^{\prime}}(u) W_{1}(g) A_{\#}^{\psi, \Upsilon}\left(M^{*} W^{\prime}, x\right)\left[\sigma(u x) W_{2}, \sigma^{\vee}(g) W_{2}^{\prime}\right]_{\sigma} d_{\psi}(x) d_{\psi}(u) d_{\psi}(g)
\end{aligned}
$$

By (3.3) and Corollary 3.1] this is equal to

$$
\omega_{\sigma}(-1) \int_{N^{\prime} \backslash G^{\prime}} \int_{N^{\prime} \backslash G^{\prime}} W_{1}(g) A_{\#}^{\psi, \Upsilon}\left(M^{*} W^{\prime}, x\right) W_{2}(x) W_{2}^{\prime}(g) d_{\psi}(x) d_{\psi}(g)=\omega_{\sigma}(-1) \cdot\left(W_{1}, W_{2}^{\prime}\right)_{\sigma}\left[W_{2}, W_{1}^{\prime}\right]_{\sigma}
$$

Therefore, Theorem 8.1 follows from the above computation and (8.4). 
8.1.1. General case. Let $\mathrm{U}_{2 n}^{-}$be the even unitary group over $F$ whose discriminant is different from that of $\mathrm{U}_{2 n}$ and dimension is $2 n$. For the convenience, we write $\mathrm{U}_{2 n}^{+}=\mathrm{U}_{2 n}$. In this section, we prove 8.1 for any discrete series representations of $\mathrm{U}_{2 n}^{ \pm}$assuming local Langlands conjecture for these groups. Indeed, the local Langlands conjecture was established by Mok 42 for $\mathrm{U}_{2 n}^{+}$and Kaletha-Minguez-Shin-White 24] for $\mathrm{U}_{2 n}^{-}$with the stabilization of the twisted trace formula established by Moeglin-Waldspurge [40, 41] assuming the weighted fundamental lemma for quasi-split groups, which is proved in Chaudouard-Laumon [9] only in the split case.

Let us briefly recall the local Langlands conjectures. Fix a splitting such that it gives Whittaker data $\left(B, \psi_{N^{\prime}}\right)$ and we denote the $L$-group of $\mathrm{U}_{2 n}^{ \pm}$by ${ }^{L} \mathrm{U}_{2 n}=\mathrm{GL}_{2 n}(\mathbb{C}) \rtimes \mathrm{Gal}(E / F)$ with the action $\theta \in \operatorname{Gal}(E / F)$ on $\mathrm{GL}_{2 n}(\mathbb{C})$ defined by $\theta(g)=w_{0}^{\prime t} g^{-1}\left(w_{0}^{\prime}\right)^{-1}$. We note that this action preserves our splitting. We also denote the connected component of ${ }^{L} \mathrm{U}_{2 n}$ by $\widehat{\mathrm{U}}_{2 n}$. The local Langlands conjecture for $\mathrm{U}_{2 n}^{+}$asserts that there exists a partition

$$
\operatorname{Irr}_{\mathrm{sqr}} \mathrm{U}_{2 n}^{+}=\coprod \Pi_{\phi}
$$

into $L$-packets, where the disjoint union on the right-hand side runs over conjugacy classes of square integrable $L$ parameters $\phi: W D_{F} \rightarrow{ }^{L} \mathrm{U}_{2 n}$. Here, we say that a continuous homomorphism $\phi: W D_{F} \rightarrow{ }^{L} \mathrm{U}_{2 n}$ is an $L$-parameter if $\phi$ is semisimple and $\left.\phi\right|_{\mathrm{SL}(2, \mathbb{C})}$ is algebraic, and that $\phi$ is square-integrable if the centralizer $S_{\phi}$ of the image of $\phi$ in $\widehat{\mathrm{U}}_{2 n}$ is finite, in which $S_{\phi}$ is an elementary abelian 2-group. Moreover, denoting by $\widehat{S}_{\phi}$ the group of characters of $S_{\phi}$, there exists an injection

$$
\Phi_{\phi} \rightarrow \widehat{S}_{\phi}, \quad \sigma \mapsto\langle\cdot, \sigma\rangle,
$$

whose image consists of the characters trivial on $\pm I_{2 n}$ and which satisfies the suitable character identity. Furthermore, the Langlands conjecture for even unitary groups asserts that there exists a partition

$$
\operatorname{Irr}_{\mathrm{sqr}} \mathrm{U}_{2 n}^{+} \amalg \operatorname{Irr}_{\mathrm{sqr}} \mathrm{U}_{2 n}^{-}=\coprod \Pi_{\phi}
$$

indexed by equivalence classes of square-integrable $L$-parameters $\phi: W D_{F} \rightarrow{ }^{L} \mathrm{U}_{2 n}$ under the conjugation by $\widehat{\mathrm{U}}_{2 n}$, and for each such $\phi$ a bijection

$$
\Phi_{\phi} \rightarrow \widehat{S}_{\phi}, \quad \sigma \mapsto\langle\cdot, \sigma\rangle,
$$

such that

$$
\Pi_{\phi}^{ \pm}:=\left\{\sigma \in \Pi_{\phi}:\left\langle-I_{2 n}, \sigma\right\rangle= \pm 1\right\}=\Pi_{\phi} \cap \operatorname{Irr}_{\mathrm{sqr}} \mathrm{U}_{2 n}^{ \pm}
$$

and the endoscopic character relations hold.

Corollary 8.1. Assume that the local Langlands conjecture holds for $\mathrm{U}_{2 n}^{ \pm}$. Then

$$
d_{\psi}^{\tilde{G}}=\left|\mathcal{S}_{\phi}\right| \lambda(E / F, \psi)^{n} \omega_{\sigma}(-1) \gamma\left(1, \sigma, \mathrm{As}^{-}, \psi\right) d_{\sigma}
$$

holds for any square-interable L-parameter $\phi: W D_{F} \rightarrow{ }^{L} \mathrm{U}_{2 n}$ and any $\sigma \in \Pi_{\phi}$.

Proof. First, we note that in the same argument as [20, p.1325], we can reduce (8.6) in the case of $\mathrm{U}_{2 n}^{-}$to the case of $\mathrm{U}_{2 n}^{+}$ using the endoscopic relations, (8.6) in the case of Steinberg representations in [18] and the proof of [49, Corollary 9.10]. Moreover, in the case of $\mathrm{U}_{2 n}^{+}$, as in the proof of [20, Corollary 5.1], we can show all representation in $\Pi_{\phi}$ have the same formal degree using the character identity. Hence, we may assume that $\langle\cdot, \sigma\rangle$ is trivial, and thus it is generic by [42, Corollary 9.2.4] (See Remark 8.1). In this case, in a similar way as the proof of [42, Corollary 9.2.4] or [3, Proposition 8.3.2], we can find quadratic extension of number fields $k^{\prime} / k$, a place $v_{0}$ of $k$, and automorphic representation $\Pi$ of $G_{2 n}\left(\mathbb{A}_{k^{\prime}}\right)$ and an irreducible globally generic cuspidal automoprhic representation $\Sigma$ of $\mathrm{U}_{2 n}\left(\mathbb{A}_{k}\right)$ such that

- $k_{v_{0}}=F, k^{\prime} \otimes k_{v_{0}}=E$

- $\Pi_{v_{0}}$ corresponds to the $L$-parameter $\iota \circ \phi$, where $\iota:{ }^{L} U_{2 n}$ is the stable base change lift

- $\Sigma$ weakly lifts to $\Pi$,

- $\Sigma_{v_{0}} \in \Pi_{\phi}$ and $\left\langle\cdot, \Sigma_{v_{0}}\right\rangle$ is trivial, i.e., $\Sigma_{v_{0}}=\sigma$

On the other hand, since the base change lift is strong by [25], we obtain

$$
\Pi_{v_{0}}=\mathrm{BC}\left(\Sigma_{v_{0}}\right) .
$$

Hence, by Theorem A.5. BC $(\sigma)$ should correspond to $\iota \circ \phi$. Then our corollary follows from Theorem 8.1 .

Remark 8.1. Atobe [4, Theorem 3.1] give a precise proof that $\sigma$ is generic if $\langle\cdot, \sigma\rangle$ is trivial using expected desideratum on the local Langlands conjecture. 
8.2. Archimedean case. In this section, we prove Conjecture 2 for discrete series representations of $U_{2 n}(\mathbb{R})$ as a consequence of the formal degree conjecture.

Lemma 8.1. Let $\phi$ be a square-integrable L-parameter of $\mathrm{U}_{2 n}(\mathbb{R})$. Then we have

$$
d_{\psi}=\left|\mathcal{S}_{\phi}\right| \lambda(E / F, \psi)^{n} \omega_{\sigma}(-1) \gamma\left(1, \sigma, \mathrm{As}^{-}, \psi\right) d_{\sigma} .
$$

Proof. By [18, Proposition 2.1], we have

$$
d_{\psi}=\left|\mathcal{S}_{\phi}\right|\left|\gamma\left(1, \sigma, \mathrm{As}^{-}, \psi\right)\right| d_{\sigma} .
$$

In this case, we may write $\phi=\oplus \chi_{i}$ with $\chi_{i}: W D_{E} \rightarrow \mathbb{C}^{\times}$. Then by a direct computation (or in a similar computation as [12, Lemma 14.2]), we see that

$$
\lambda(E / F, \psi)^{n} \omega_{\sigma}(-1) \gamma\left(1, \sigma, \mathrm{As}^{-}, \psi\right)
$$

is positive real number with the absolute value $\left|\gamma\left(1, \sigma, \mathrm{As}^{-}, \psi\right)\right|$. Thus, our required identity holds.

Theorem 8.2. Conjecture 2 for any generic discrete series representations of $\mathrm{U}_{2 n}(\mathbb{R})$.

Proof. Suppose $\sigma \in \operatorname{Irr}_{\text {sqr, gen }}$. Then $\mathrm{BC}(\sigma)=\chi_{1} \times \cdots \chi_{2 n}$ where $\chi_{i}$ are mutually different unitary characters of $\mathbb{C}^{\times}$. Then we can find irreducible automorphic representations $\Pi_{i}$ of $\mathbb{A}_{\mathbb{Q}(i)}$ such that $\Pi_{i, \infty}=\chi_{i}$. Set $\Pi=\Pi_{1} \times \cdots \Pi_{2 n}$. We see that $\sigma$ is good in the sense of [29, 5.3] since $\Pi_{\infty}=\mathrm{BC}(\sigma)$. In particular, there exists $c_{\sigma}$ such that

$$
\int_{N^{\prime}}^{s t} J\left(W, W^{\prime}, \frac{1}{2}\right) \psi_{N^{\prime}}(n) d n=\omega_{\sigma}(-1) W(e) A_{\#}^{\psi, \Upsilon}\left(M^{*} W^{\prime}, e\right)
$$

for any $W \in \mathbb{W}^{\psi^{\prime}}-1(\sigma)$ and $W^{\prime} \in \operatorname{Ind}\left(\mathbb{W}^{\psi_{N_{M}}}(\pi)\right)$. Then the same argument as the proof of Theorem 8.1 gives

$$
c_{\pi}=\omega_{\sigma}(-1) \Longleftrightarrow d_{\psi}=2^{k} \lambda(E / F, \psi)^{n} \omega_{\sigma}(-1) \gamma\left(1, \mathfrak{c}(\pi), \mathrm{As}^{-}, \psi\right) d_{\sigma},
$$

where we have used the following in the real case.

(1) The integral $J$ converges absolutely uniformly near $s=-\frac{1}{2}$ (cf. Remark 3.3).

(2) Any Whittaker function $W \in \mathbb{W}^{\psi_{N^{\prime}}^{-1}}(\sigma)$ is square-integrable over $N^{\prime} \backslash G^{\prime}$ (cf. [53, Theorem 15.3.4]).

(3) Theorem A.4 $\mathrm{U}_{2 n}(\mathbb{R})$ readily follows from the Langlands correspondence.

(4) We can prove the absolute convergence of the double integral (8.5) in the same argument as [20, p.1316-1317] using [52, Theorem 7.2.1], [53, Theorem 15.2.4] and Lemma 3.5]

\section{Appendix A. On the image of LOCAL BASE Change Lifts For GENERIC REPRESEntations}

In this appendix, using a similar argument as Jiang and Soudy 22] and Liu [35, we shall determine the image of local base change lifts for generic discrete series representations of even unitary groups $U_{2 n}$. We note that as in the above two papers, we may write down Langlands parameter corresponding to these representations. We will consider this problem for not only even unitary groups but also odd unitary groups in our future work.

In this appendix, we will use the same notation in the main body of this paper. Further, for simplicity, we say that a representation of $\mathrm{U}_{2 n}$ is generic if it is $\psi_{N}$-generic. For a given representations $\tau_{i}$ of $\mathrm{GL}_{k_{i}}(E), i=1, \cdots, a$ and $\rho$ of $\mathrm{U}_{2 m}$, we denote by

$$
\tau_{1} \times \cdots \times \tau_{k_{a}} \quad\left(\text { resp. } \tau_{1} \times \cdots \times \tau_{k_{a}} \times \rho\right)
$$

the parabolic induction of $\mathrm{GL}_{k_{1}+\cdots+k_{a}}$ (resp. $\left.\mathrm{U}_{2\left(k_{1}+\cdots+k_{a}+m\right)}\right)$ for the parabolic subgroup with the Levi part $\mathrm{GL}_{k_{1}}(E) \times$ $\cdots \times \mathrm{GL}_{k_{1}}(E)\left(\right.$ resp. $\left.\mathrm{GL}_{k_{1}}(E) \times \cdots \times \mathrm{GL}_{k_{1}}(E) \times \mathrm{U}_{2 m}\right)$.

We would like to start our observation with the case of supercuspidal representations. Let $\Pi^{(s g)}\left(\mathrm{U}_{2 n}\right)$ be the set of all equivalence classes of irreducible supercuspidal generic representations of $\mathrm{U}_{2 n}$. Let $\Pi^{(s g)}\left(\mathrm{GL}_{2 n}(E)\right)$ be the set of all equivalence classes of irreducible tempered representations of $\mathrm{GL}_{2 n}(E)$ of the form

$$
\tau_{1} \times \cdots \times \tau_{r}
$$

where $\tau_{i}$ is an irreducible supercuspidal representation of $\mathrm{GL}_{n_{i}}(E)$ such that $L\left(s, \tau_{i}, \mathrm{As}^{+}\right)$has a pole at $s=0$ and for $i \neq j$, $\tau_{i} \nsucceq \tau_{j}$. In 25, Kim and Krishnamurthy constructed local base change lifts of generic representations of $\mathrm{U}_{2 n}$ explicitly. Indeed, we have the following result.

Theorem A.1 (Proposition 8.4 in [25]). There is a map $l$ from $\Pi^{(s g)}\left(\mathrm{U}_{2 n}\right)$ to $\Pi^{(s g)}\left(\mathrm{GL}_{2 n}(E)\right)$ which preserves local $\gamma$-factors with GL-twist, namely

$$
\gamma^{S h}(s, \pi \times \sigma, \psi)=\lambda(E / F, \psi)^{2 n k} \gamma^{R S}(s, l(\pi) \times \sigma, \psi)
$$

for any $\pi \in \Pi^{(s g)}\left(\mathrm{U}_{2 n}\right)$ and any irreducible generic representation $\sigma$ of $\mathrm{GL}_{k}(E)$ with $k \in \mathbb{N}$. Here, the $\gamma$-factor on the right-hand (res. left-hand) side is defined by the Rankin-Selberg method [21] (resp. Langlands-Shahidi method [48, and [49]). 
Recently, the author proved the following result.

Theorem A.2 (Corollary 9.2 in [43). The map $l$ in Theorem A.1 is bijective and unique.

Let us extend the above result to the case of generic discrete series representations. Let us recall the construction of generic discrete series representations by Moeglin and Tadić 38. To explain their construction, let us define some representations of general linear groups. Let $\rho$ be an irreducible supecuspidal representation of $\mathrm{GL}_{k}(E)$. Then for integers $l \geq m>0$ with the same parity, we write by $D(l, m, \rho)$ the unique irreducible subrepersentation of

$$
\operatorname{Ind}\left(|\operatorname{det}|^{(l-1) / 2} \rho \otimes|\operatorname{det}|^{(l-1) / 2-1} \rho \otimes \cdots \otimes|\operatorname{det}|^{-(m-1) / 2} \rho\right)
$$

and by $D(l, \rho)$ the unique subrepresentation of

$$
\operatorname{Ind}\left(|\operatorname{det}|^{(l-1) / 2} \rho \otimes|\operatorname{det}|^{(l-1) / 2-1} \rho \otimes \cdots \otimes|\operatorname{det}|^{(l+1) / 2-[l / 2]} \rho\right) .
$$

Then $D(l, m, \rho)$ (resp. $D(l, \rho))$ is essentially square integrable representation of $\operatorname{GL}_{\frac{k(l+m)}{2}}(E)\left(\right.$ resp. GL $\left.\left[\frac{l}{2}\right] k(E)\right)$. Further, we write

$$
S t(\rho, l)=D(l, l, \rho)
$$

Then we consider a parabolic induction

$$
D\left(l_{1}, m_{1}, \rho_{1}\right) \times \cdots \times D\left(l_{r}, m_{r}, \rho_{r}\right) \times D\left(l_{r+1}, \rho_{r+1}\right) \times \cdots \times D\left(l_{t}, \rho_{t}\right) \times \tau_{0}
$$

where $l_{i}>m_{i}>0, \tau_{0}$ is an irreducible supercuspidal representation of a smaller even unitary group and $\rho_{i}$ are irreducible supercuspidal representation of $\mathrm{GL}_{k_{i}}(E)$ such that $\rho_{i}$ is conjugate self-dual. Further, we shall suppose that

$$
L\left(s, \rho_{i}, \mathrm{As}^{+}\right) \text {has a pole at } s=0 \text { if and only if } l_{i} \text { is odd. }
$$

Since $L\left(s, \rho_{i}, \mathrm{As}^{+}\right)$has a pole at $s=0$ if and only if $\rho_{i}$ is $\mathrm{GL}_{k_{i}}(F)$-distinguished by Anandavardhanan-Kable-Tandon 2 , Corollary 1.5], the above condition is equivalent to that

$$
\rho_{i} \text { is } \mathrm{GL}_{k_{i}}(F) \text {-distinguished if and only if } l_{i} \text { is odd. }
$$

We know that $\rho_{i}$ should be $\left(\mathrm{GL}_{k_{i}}(F), \eta_{E / F}\right)$-distinguished or $\mathrm{GL}_{k_{i}}(F)$-distinguished by Kable [23, Theorem]. Moreover if $\rho_{i}$ is $\left(\mathrm{GL}_{k_{i}}(F), \eta_{E / F}\right)$-distinguished, $\rho_{i}$ is not $\mathrm{GL}_{k_{i}}(F)$-distinguished, and vice versa by [2, Corollary 1.6]. When the above condition holds, the unique generic constituent of the above induced representation is discrete series representation. When it is a representation of $\mathrm{U}_{2 n}$, every discrete series representation is obtained in this way. Let $\Pi^{(d g)}\left(\mathrm{U}_{2 n}\right)$ be the set of irreducible generic discrete series representations of $\mathrm{U}_{2 n}$, namely the set of all irreducible representation obtained in the above way.

Let $\Pi^{(d g)}\left(\mathrm{GL}_{2 n}(E)\right)$ be the set of irreducible representations of $\mathrm{GL}_{2 n}(E)$ of the form $\pi=\tau_{1} \times \cdots \times \tau_{r}$ where $\tau_{i}$ is an irreducible discrete series representation of $\mathrm{GL}_{n_{i}}(E)$ such that $L\left(s, \tau_{i}, \mathrm{As}^{+}\right)$has a pole at $s=0$. We note that $\tau_{i} \not \tau_{j}$ if $i \neq j$ because of the irreducibility of $\pi$. Further, we know that $\tau_{i}$ is conjugate self-dual, in particular it is unitary. Then by Bernstein-Zelevinsky [8, we may write

$$
\tau_{i}=\operatorname{St}\left(\rho_{i}, a_{i}\right)
$$

where $a_{i} \in \mathbb{Z}_{\geq 0}$ and $\rho_{i}$ is an irreducible supercuspidal representation of $\mathrm{GL}_{m_{i}}(E)$ such that $n_{i}=a_{i} m_{i}$. We have a necessary and sufficient condition for $\operatorname{St}\left(\rho_{i}, a_{i}\right)$ to be $\operatorname{GL}_{n_{i}}(F)$-distinguished.

Theorem A.3 (Corollary 4.2 in [36]). Let $\rho$ be an irreducible supercuspidal representation of $\mathrm{GL}_{k}(E)$ and a $\in \mathbb{N}$. Then generalized Seinberg representation $\operatorname{St}(\rho, a)$ of $\mathrm{GL}_{a k}(E)$ is $\mathrm{GL}_{a k}(F)$-distinguished if and only if $\rho$ is $\left(\mathrm{GL}_{k}(F), \eta_{E / F}^{a-1}\right)$ distinguished.

From this theorem, we see that $\rho_{i}$ is $\eta_{E / F}^{a_{i}-1}$-distinguished for any $i$. In particular, $\rho_{i}$ is conjugate self-dual. Then we prove the following generalization of Theorem A.1, A.2 to discrete series representations.

Theorem A.4. There is a bijective map l from $\Pi^{(d g)}\left(\mathrm{U}_{2 n}\right)$ to $\Pi^{(d g)}\left(\mathrm{GL}_{2 n}(E)\right)$ satisfying the condition

$$
\gamma^{S h}(s, \pi \times \sigma, \psi)=\lambda(E / F, \psi)^{2 n k} \gamma^{R S}(s, l(\pi) \times \sigma, \psi)
$$

for any $\pi \in \Pi^{(d g)}\left(\mathrm{U}_{2 n}\right)$ and any irreducible generic representation $\sigma$ of $\mathrm{GL}_{k}(E)$ with $k \in \mathbb{N}$. Further, the above map is unique.

Proof. First, we note that the uniqueness follows from a local converse theorem for generic representations of $\mathrm{GL}_{2 n}(E)$ by Henniart [17. Following Kim-Krishnamurthy [25, we define a map from $\Pi^{(d g)}\left(\mathrm{U}_{2 n}\right)$ to $\Pi^{(d g)}\left(\mathrm{GL}_{2 n}(E)\right)$. Let $\pi$ be an element of $\Pi^{(d g)}\left(\mathrm{U}_{2 n}\right)$, and write it as (A.1). Then we define $l(\pi)$ by

$$
\operatorname{St}\left(\rho_{1}, l_{1}\right) \times \operatorname{St}\left(\rho_{1}, m_{1}\right) \times \cdots \times \operatorname{St}\left(\rho_{r}, l_{r}\right) \times \operatorname{St}\left(\rho_{r}, m_{r}\right) \times \operatorname{St}\left(\rho_{r+1}, l_{r+1}\right) \times \cdots \times \operatorname{St}\left(\rho_{t}, l_{t}\right) \times l\left(\tau_{0}\right),
$$

which is irreducible by Bernstein-Zelevinsky [8]. First, let us check that this representation is in $\Pi^{(d g)}\left(\mathrm{GL}_{2 n}(E)\right)$. From Theorem A.1 $l\left(\tau_{0}\right)$ should be of the form $\Pi_{1} \times \cdots \times \Pi_{u}$ where $\Pi_{i}$ are mutually distinct irreducible supercuspidal representations such that $L\left(s, \Pi_{i}, \mathrm{As}^{+}\right)$has a pole at $s=0$. Thus, it suffices to check that $\operatorname{St}\left(\rho_{i}, l_{i}\right)$ is $\operatorname{GL}(F)$-distinguished. Indeed, 
$\operatorname{St}\left(\rho_{i}, l_{i}\right)$ is $\mathrm{GL}_{l_{i} k_{i}}(F)$-distinguished if and only if $\rho_{i}$ is $\left(\mathrm{GL}(F), \omega_{E / F}^{l_{i}-1}\right)$-distinguished by Theorem A.3 From the condition (A.2), if $l_{i}$ is odd (resp. even), $\rho_{i}$ is $\operatorname{GL}_{k_{i}}(F)$-distinguished (resp. $\left(\mathrm{GL}_{k_{i}}(F), \omega_{E / F}^{l_{i}-1}\right)$-distinguished). Thus, $\operatorname{St}\left(\rho_{i}, l_{i}\right)$ is $\mathrm{GL}_{l_{i} k_{i}}(F)$-distinguished.

From the definition of $\Pi^{(d g)}\left(\mathrm{GL}_{2 n}(E)\right)$, the surjectivity of the map $l$ is clear. Further, from the local converse theorem [43, Theorem 9.4] for $\mathrm{U}_{2 n}$, its injectivity follows.

Finally, we shall realize the above map by the local descent.

Theorem A.5. The map $\pi \mapsto \mathcal{D}_{\psi}^{\Upsilon}(\pi)$ defines a bijection

$$
\mathcal{D}_{\psi}^{\Upsilon}: \Pi^{(d g)}\left(\mathrm{GL}_{2 n}(E)\right) \rightarrow \Pi^{(d g)}\left(\mathrm{U}_{2 n}\right)
$$

Moreover, if $\pi \in \Pi^{(d g)}\left(\mathrm{GL}_{2 n}(E)\right)$ and $\tilde{\pi}=\mathcal{D}_{\psi}^{\Upsilon}(\pi \otimes \Upsilon)$ then

$$
\gamma(s, \tilde{\pi} \times \tau, \psi)=\lambda(E / F)^{2 n k} \gamma(s, \pi \times \tau, \psi) .
$$

for any irreducible generic representation $\tau$ of $\mathrm{GL}_{k}(E)$.

Proof. First, we shall prove that $\mathcal{D}_{\psi}^{\Upsilon}(\pi)$ is irreducible. From Theorem A.4 there is an irreducible discrete series representation $\sigma$ of $G^{\prime}$ such that $l(\sigma)=\pi$ where $l$ is the map constructed in that theorem. Indeed, from its definition and explicit description of local base change lift by Kim-Krishnamurthy [25, $l(\sigma)=\mathrm{BC}(\sigma)$. Let us take number fields $L / K$ such that for some place $v_{0}$ of $K, L_{v_{0}} \simeq E$ and $K_{v_{0}} \simeq F$. If necessary, replacing $\psi$ by $\psi^{a}$ with some $a \in\left(F^{\times}\right)^{2}$, we may suppose that $\psi$ is $v_{0}$-component of an additive character $\psi_{\mathbb{A}_{K}}$ of $\mathbb{A}_{K}$. Then by [20, Corollary A.6], there is $\psi_{\mathbb{A}_{K}}$-generic irreducible cuspidal automorphic representation $\Sigma$ of $\mathrm{U}_{2 n}\left(\mathbb{A}_{K}\right)$ such that $\Sigma_{v_{0}}=\sigma$. From the explicit construction of base change lifts, we have $\mathrm{BC}(\Sigma)_{v_{0}}=\mathrm{BC}(\sigma)=\pi$. Let us take a character $\eta$ of $\mathbb{A}_{L}^{\times}$such that its restriction to $\mathbb{A}_{E}^{\times}$is the quadratic character $\omega_{L / K}$ corresponding to $L / K$ and $\eta_{v_{0}}=\Upsilon$. Then by [29], $\mathcal{D}_{\psi_{\mathbb{A}_{K}}}^{\eta}(\mathrm{BC}(\Sigma))_{v_{0}}=\mathcal{D}_{\psi}^{\Upsilon}(\pi)$ is irreducible.

Second of all, we shall prove $\mathrm{BC}\left(\mathcal{D}_{\psi}^{\Upsilon}(\pi)\right) \in \Pi^{(d g)}\left(\mathrm{GL}_{2 n}\right)$. From the above argument, we have a globalization $\Pi:=\mathrm{BC}(\Sigma)$ of $\pi$. Further, from the unramified computation and strong multiplicity one theorem for $\mathrm{GL}_{2 n}$, we get $\mathrm{BC}\left(\mathcal{D}_{\psi_{\mathbb{A}_{K}}}^{\eta}(\Pi)\right)=$ $\Pi \otimes \eta^{-1}$ and thus $\operatorname{BC}\left(\mathcal{D}_{\psi_{\AA_{K}}}^{\eta}(\Pi)\right)_{v_{0}}=\pi \otimes \Upsilon^{-1}$. From the construction of base change lifts, we find that $\operatorname{BC}\left(\mathcal{D}_{\psi_{\AA_{K}}}^{\eta}(\Pi)\right)_{v_{0}}=$ $\operatorname{BC}\left(\left(\mathcal{D}_{\psi_{\mathbb{A}_{K}}}^{\eta}(\Pi)\right)_{v_{0}}\right)$. From the definition of explicit local descent, we get $\left(\mathcal{D}_{\psi_{A_{K}}}^{\eta}(\Pi)\right)_{v_{0}}=\mathcal{D}_{\psi}^{\Upsilon}\left(\Pi_{v_{0}}\right)$. Therefore,

$$
\operatorname{BC}\left(\mathcal{D}_{\psi}^{\Upsilon}(\pi)\right)=\pi \otimes \Upsilon^{-1} \in \Pi^{(d g)}\left(\mathrm{GL}_{2 n}\right)
$$

Third of all, we prove $\mathcal{D}_{\psi}^{\Upsilon}(\pi) \in \Pi^{(d g)}\left(G^{\prime}\right)$. From Theorem A.4 and the previous claim, there is $\sigma^{\prime} \in \Pi^{(d g)}\left(G^{\prime}\right)$ such that $l\left(\sigma^{\prime}\right)=\operatorname{BC}\left(\mathcal{D}_{\psi}^{\Upsilon}(\pi)\right)$. The base change lift is strong, so that $\gamma\left(s, \operatorname{BC}\left(\mathcal{D}_{\psi}^{\Upsilon}(\pi)\right) \times \tau, \psi\right)=\lambda(E / F)^{n} \gamma\left(s, \mathcal{D}_{\psi}^{\Upsilon}(\pi) \times \tau, \psi\right)$ for any irreducible generic representation $\tau$ of $\mathrm{GL}_{i}$ with $1 \leq i \leq 2 n$. Again, by Theorem A.4 $\gamma\left(s, l\left(\sigma^{\prime}\right) \times \tau, \psi\right)=\lambda(E /$ $F)^{n} \gamma\left(s, \sigma^{\prime} \times \tau, \psi\right)$. Hence,

$$
\gamma\left(s, \mathcal{D}_{\psi}^{\Upsilon}(\pi) \times \tau, \psi\right)=\gamma\left(s, \sigma^{\prime} \times \tau, \psi\right)
$$

and the local converse theorem [43, Theorem 9.4] implies that

$$
\mathcal{D}_{\psi}^{\Upsilon}(\pi)=\sigma \in \Pi^{(d g)}\left(\mathrm{U}_{2 n}\right) .
$$

Further we note that if $\mathcal{D}_{\psi}^{\Upsilon}\left(\pi_{1}\right)=\mathcal{D}_{\psi}^{\Upsilon}\left(\pi_{2}\right)$, then $\pi_{1} \simeq \pi_{2}$ by (A.3).

Finally, we shall prove $l(\pi)=\mathcal{D}_{\psi}^{\Upsilon}(\pi)$. Since the base change is strong, we have $\lambda(E / F)^{2 n k} \gamma\left(s, \mathrm{BC}\left(\mathcal{D}_{\psi}^{\Upsilon}(\pi)\right) \times \tau, \psi\right)=$ $\gamma\left(s, \mathcal{D}_{\psi}^{\Upsilon}(\pi) \times \tau, \psi\right)$. On the other hand, we have $\gamma\left(s, \operatorname{BC}\left(\mathcal{D}_{\psi}^{\Upsilon}(\pi)\right) \times \tau, \psi\right)=\gamma\left(s, \pi \otimes \Upsilon^{-1} \times \tau, \psi\right)$ by (A.3). Hence, we get

$$
\lambda(E / F)^{2 n k} \gamma(s, \pi \times \tau, \psi)=\gamma\left(s, \mathcal{D}_{\psi}^{\Upsilon}(\pi \otimes \Upsilon) \times \tau, \psi\right) .
$$

Then our assertion follows from the uniqueness in Theorem A.4.

\section{ApPendix B. Stability of CERTAin OSCILlatory INTEGRALS FOR QUASI-SPlit REDUCTIVE GROUPS AND NON-VANISHING OF BESSEL FUNCTIONS}

In this section, we shall prove the stability of certain oscillatory integrals for a quasi-split reductive group $\mathbf{G}$ over a non-archimedean local field $F$ of characteristic zero. This is a generalization of 28] to quasi-split reductive groups.

B.1. main results. Denote $G=\mathbf{G}(F)$. Fix a Borel subgroup $B$ of $G$, and we denote its unipotent radical by $N$. Let $\psi_{N}$ be a nondegenerate character of $N$. Consider the space $\Omega\left(N \backslash G, \psi_{N}\right)$ of smooth functions on $G$ such that $f(n g)=\psi_{N}(n) f(g)$ for all $n \in N, g \in G$. Denote the regular representation of $G$ on $\Omega\left(N \backslash G, \psi_{N}\right)$ by $R$. For any compact open subgroup $K$ of $G$ let $\Omega\left(N \backslash G, \psi_{N}\right)^{K}$ denote the subspace of right $K$-invariant functions. Let $\overline{w_{0}} \in G$ be a rerresentative of the longest Weyl element $w_{0}$. Fix a Haar measure on $N$. For any compact open subgroup $N^{\prime}$ of $N$ and $W \in \Omega\left(N \backslash G, \psi_{N}\right)$ let

$$
R_{N^{\prime}, \psi_{N}} W:=\frac{1}{\operatorname{vol}\left(N^{\prime}\right)} \int_{N^{\prime}}\left(R\left(n^{\prime}\right) W\right) \psi_{N}\left(n^{\prime}\right)^{-1} d n^{\prime} \in \Omega\left(N \backslash G, \psi_{N}\right) .
$$


Theorem B.1. For any open subgroup $K$ of $G$, there exists an open compact subgroup $N^{\prime}$ of $N$ such that, for any $W \in \Omega\left(N \backslash G, \psi_{N}\right)^{K},\left(R_{N^{\prime}, \psi_{N}} W\right)\left(\overline{w_{0}}.\right)$ is compactly supported on $N$.

As a consequence. for any $W \in \Omega\left(N \backslash G, \psi_{N}\right)$ and a compact open subgroup $N^{\prime}$ of $N$ satisfying the above condition, we can define the stable integral

$$
\int_{N}^{s t} W\left(\overline{w_{0}} n\right) \psi_{N}(n)^{-1} d n:=\int_{N}\left(R_{N^{\prime}, \psi_{N}} W\right)\left(\overline{w_{0}} n\right) \psi_{N}(n)^{-1} d n .
$$

More generally, we say that $g \in G$ is relevant (with respect to $\psi_{N}$ ), if $\psi_{N}\left(g n g^{-1}\right)=\psi_{N}(n)$ for all $n \in N \cap g^{-1} N g$. For any $g \in G_{\text {rel }}$ which is the set of relevant elements, we may use the above procedure to regularize the integral.

Theorem B.2. For any open subgroup $K$ of $G$ and $g \in G$, there exists an open compact subgroup $N^{\prime}$ of $N$ sucht that, for any $W \in \Omega\left(N \backslash G, \psi_{N}\right)^{K},\left(R_{N^{\prime}, \psi_{N}} W\right)(g \cdot)$ is compactly supported on $g^{-1} N g \cap N \backslash N$. In particular, when $g \in G_{\mathrm{rel}}$, we can define

$$
\int_{g^{-1} N g \cap N \backslash N} W(g n) \psi_{N}(n)^{-1} d n:=\int_{g^{-1} N g \cap N \backslash N}\left(R_{N^{\prime}, \psi_{N}} W\right)(g n) \psi_{N}(n)^{-1} d n
$$

where $N^{\prime}$ is a compact open subgroup of $N$ given in Theorem B.1.

Remark B.1. Let $\pi$ be a $\psi_{N}$-generic irreducible admissible representation of $G$. Then by the uniqueness of Whittaker functionals, there is a function $\mathbb{B}_{\pi}^{\psi_{N}}$ on $G$ such that

$$
\int_{g^{-1} N g \cap N \backslash N} W(g n) \psi_{N}(n)^{-1} d n=\mathbb{B}_{\pi}^{\psi_{N}}(g) W(e)
$$

for $g \in G_{\mathrm{rel}}$ and $\mathbb{B}_{\pi}^{\psi_{N}}(g)=0$ for $g \in G \backslash G_{\mathrm{rel}}$. Then function $\mathbb{B}_{\pi}^{\psi_{N}}$ is called the Bessel function attached to $\pi$.

B.2. Notation. Suppose that $\mathbf{G}$ is a semisimple quasi-split group defined over $F$ of rank $r$ since main results are easily reduced to the semisimple case. Write $G=\mathbf{G}(F)$. Let $B$ be a Borel subgroup of $G, T$ a maximal torus contained in $B$, $A \subset T$ a maximal $F$-split torus of $G$ and $N$ be the unipotent radical of $B$ so that $B=T N$. Let $\bar{B}=T \bar{N}$ be the opposite Borel subgroup with respect to $T$. Let $\mathbf{K}$ be a hyper special maximal compact open subgroup of $G$ in good position with respect to $B$. Then we have the Iwasawa decomposition $G=T N \mathbf{K}$. Let $X^{*}(Y)$ (resp. $\left.X_{*}(Y)\right)$ be the lattice of rational characters (resp. cocharacters) of a group $Y$.

B.2.1. Relative roots and weights. Let $\Phi \subset X^{*}(T)$ be the set of root of $T$ in $\mathfrak{g}:=\operatorname{Lie}(G)$. Further, let $\Phi_{\text {rel }} \subset X^{*}(A)$ be the set of root of $A$ in $\mathfrak{g}, \Phi_{\text {rel, }+}$ be the subset of positive roots in $\Phi_{\text {rel }}$ and $\triangle_{0}$ be the subset of simple roots with respect to $(B, A)$ in $\Phi_{\text {rel }}$. Similarly, let $\triangle_{0}^{\vee} \subset \Phi_{\text {rel, },}^{\vee} \subset \Phi_{\text {rel }}^{\vee} \subset X_{*}(A)$ be the sets of (simple, or positive) co-roots. For $\alpha \in X^{*}(A)$, we denote by $\mathfrak{g}_{\alpha}$ the $\alpha$-eigenspace in $\mathfrak{g}$. We denote by $\alpha \leftrightarrow \alpha^{\vee}$ the canonical bijection between $\Phi_{\text {rel }}$ and $\Phi_{\text {rel }}^{\vee}$ (resp. $\Phi_{\mathrm{rel},+} \leftrightarrow \Phi_{\mathrm{rel},+}^{\vee}$ ). Denote $\mathfrak{a}_{T}:=X_{*}(T) \otimes \mathbb{R}$.

For $\alpha \in \Phi_{\text {rel }}$, we denote by $N_{\alpha}$ the unipotent subgroup whose Lie algebra is $\mathfrak{g}_{\alpha}+\mathfrak{g}_{2 \alpha}$. For $\alpha \in \triangle_{0}$, let $P_{\alpha}$ be the standard parabolic subgroup with respect to $\alpha$. Then its Levi subgroup $M_{\alpha}$ has rank 1, so does the simply connected covering $\tilde{M}_{\alpha}$. Then, $\tilde{M}_{\alpha}$ is $\mathrm{SL}_{2}\left(F_{\alpha}\right)$ or the quasi-split special unitary group $\mathrm{SU}(3)_{\alpha}$ with respect to a quadratic extension $E_{\alpha}$ of $F_{\alpha}$ where $F_{\alpha}$ is a finite extension of $F$. We say that $\alpha \in \triangle_{0}$ is of type (I) (resp. type (II)) if $\tilde{M}_{\alpha}$ is isomorphic to $\mathrm{SL}_{2}\left(F_{\alpha}\right)$ (resp. $\left.\mathrm{SU}(3)_{\alpha}\right)$.

Suppose that $\alpha \in \triangle_{0}$ is of type (II). Then we may realize $\mathrm{SU}(3)_{\alpha}$ by

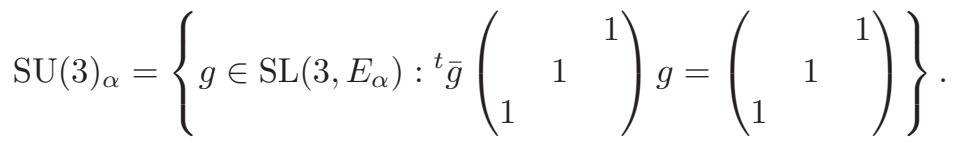

Further, we may take the following group as a Borel subgroup of $\mathrm{SU}(3)_{\alpha}$ :

$$
\tilde{B}_{\alpha}=\tilde{T}_{\alpha} \tilde{N}_{\alpha}
$$

where

$$
\tilde{T}_{\alpha}=\left\{t_{\alpha}(y):=\left(\begin{array}{ccc}
y & & \\
& y / \bar{y} & \\
& & \bar{y}^{-1}
\end{array}\right): y \in E_{\alpha}^{\times}\right\}
$$

and

$$
\tilde{N}_{\alpha}=\left\{x_{\alpha}(r, m):=\left(\begin{array}{ccc}
1 & r & m \\
& 1 & -\bar{r} \\
& & 1
\end{array}\right): x, y \in E_{\alpha} \text { s.t. } N_{E_{\alpha} / F_{\alpha}}(r)=-\operatorname{Tr}_{E_{\alpha} / F_{\alpha}}(m)\right\}
$$

Here, $N_{E_{\alpha} / F_{\alpha}}$ (resp. $\operatorname{Tr}_{E_{\alpha} / F_{\alpha}}$ ) is the norm (resp. trace) map from $E_{\alpha}$ to $F_{\alpha}$. Note that

$$
x_{\alpha}(r, m)^{-1}=x_{\alpha}(-r, \bar{m})
$$


Hereafter, we fix a covering map of $M_{\alpha}$, namely

$$
1 \rightarrow \pi_{1}\left(M_{\alpha}\right) \rightarrow \tilde{M}_{\alpha} \stackrel{f_{\alpha}}{\longrightarrow} M_{\alpha} \rightarrow 1
$$

so that $f_{\alpha}\left(\tilde{T}_{\alpha}\right)=T_{\alpha}:=T \cap M_{\alpha}$ and $f_{\alpha}\left(\left\{x_{\alpha}(r, m): r, m \in \mathcal{O}_{E_{\alpha}}\right\}\right)=N_{\alpha} \cap \mathbf{K}$ where $\mathcal{O}_{E_{\alpha}}$ denotes the ring of integers of $E_{\alpha}$. Further, we denote the image of $t_{\alpha}(y)$ and $x_{\alpha}(r, m)$ by the same symbol. Then $N_{\alpha}$ is the unipotent subgroup of $G$ generated by $\left\{x_{\alpha}(r, m): r, m \in E_{\alpha}\right.$ such that $\left.N_{E_{\alpha} / F_{\alpha}}(r)=-\operatorname{Tr}_{E_{\alpha} / F_{\alpha}}(m)\right\}$. For $\alpha \in \triangle_{0}$, write $N^{\alpha}$ for the unipotent radical of the parabolic subgroup $B \cup B s_{\alpha} B$. Then we may write

$$
N=N_{\alpha} \ltimes N^{\alpha}
$$

Further, for $r, m \in E_{\alpha}$ such that $N_{E_{\alpha} / F_{\alpha}}(r)=-\operatorname{Tr}_{E_{\alpha} / F_{\alpha}}(m)$, we define

$$
x_{-\alpha}(r, m):=\left(\begin{array}{ccc}
1 & & \\
-\bar{r} & 1 & \\
m & r & 1
\end{array}\right)
$$

Also, we write the image of this element by the same symbol. From the definition, we can take the co-character $\alpha^{\vee}$ so that

$$
\alpha^{\vee}(x)=\left(\begin{array}{ccc}
x & & \\
& 1 & \\
& & x^{-1}
\end{array}\right), \quad x \in F^{\times}
$$

Further, we extend $\alpha^{\vee}$ to $E_{\alpha}^{\times} \rightarrow T_{\alpha}$ by

$$
\alpha^{\vee}(x)=\left(\begin{array}{ccc}
x & & \\
& \bar{x} / x & \\
& & \bar{x}^{-1}
\end{array}\right), \quad x \in E_{\alpha}^{\times} .
$$

More generally, for $\beta=w \alpha$ with $w \in \mathbf{W}$ and $\alpha \in \triangle_{0}$ of type (II), we define

$$
x_{\beta}(r, m):=w x_{\alpha}(r, m) w^{-1} \text { and } \beta^{\vee}:=w \cdot\left(\alpha^{\vee}\right)
$$

Suppose that $\alpha \in \triangle_{0}$ is of type (I). Then we choose a parametrization $n_{\alpha}: F_{\alpha} \rightarrow N_{\alpha}$ such that $n_{\alpha}\left(\mathcal{O}_{F_{\alpha}}\right)=N_{\alpha} \cap \mathbf{K}$. Here, $\mathcal{O}_{F_{\alpha}}$ denotes the ring of integers of $F_{\alpha}$. Further, we define

$$
\alpha^{\vee}(x)=\left(\begin{array}{cc}
x & \\
& x^{-1}
\end{array}\right), \quad x \in F^{\times}
$$

and we also extend $\alpha^{\vee}$ to $F_{\alpha}^{\times} \rightarrow T_{\alpha}$ by

$$
\alpha^{\vee}(x)=\left(\begin{array}{cc}
x & \\
& x^{-1}
\end{array}\right), \quad x \in F_{\alpha}^{\times} .
$$

Similarly as above, for $\beta=w \alpha$ with $w \in \mathbf{W}$ and $\alpha \in \triangle_{0}$ of type (I), we define

$$
n_{\beta}(x):=w x_{\alpha}(x) w^{-1} \quad \text { and } \quad \beta^{\vee}:=w \cdot\left(\alpha^{\vee}\right) .
$$

On the other hand, let $\lambda=\sum_{\alpha \in \triangle_{0}} r_{\alpha} \alpha \in \mathfrak{a}_{0}^{*}$, and define a character a character $|\lambda|: A \rightarrow \mathbb{R}_{+}$by

$$
\lambda(t)=\prod_{\alpha \in \triangle_{0}}|\alpha(t)|^{r_{\alpha}}
$$

For $\alpha \in \triangle_{0}$, we may consider $\alpha$ as a character of $\alpha^{\vee}\left(F_{\alpha}^{\times}\right)$in the trivial way. Further, when $\alpha$ is of type (II), we may extend a character $|\alpha|$ to a character $\left|\alpha_{0}\right|: T_{\alpha_{i}} \rightarrow \mathbb{R}_{+}$, and naturally a character of $T$. Indeed, we define

$$
\left|\alpha_{0}\right|\left(\alpha^{\vee}(x)\right)=|\alpha|\left(\alpha^{\vee}\left(N_{E_{\alpha} / F_{\alpha}}(x)\right)\right)^{1 / 2} .
$$

Therefore, $\lambda=\sum_{\alpha \in \triangle_{0}} r_{\alpha} \alpha$, we can define $\left|\lambda_{0}\right|: T \rightarrow \mathbb{R}_{+}$by $\left|\lambda_{0}\right|:=\prod_{\alpha \in \triangle_{0}}\left|\alpha_{0}\right|^{r_{\alpha}}$.

By the Iwasawa decomposition, we extend $\left|\lambda_{0}\right|$ to a left- $N$ and right-K invariant function on $G$. For any compact subset $C \subset G$, there exists a constant $\kappa_{C}$ (depending also on $\lambda$ ) such that

$$
\kappa_{C}^{-1}\left|\lambda_{0}\right|(g) \leq\left|\lambda_{0}\right|(g h) \leq \kappa_{C}\left|\lambda_{0}\right|(g)
$$

for all $g \in G$ and $h \in C$.

Let $H: T \rightarrow \mathfrak{a}_{T}$ be the Harish-Chandra map given by

$$
\exp \langle\chi, H(t)\rangle=|\chi(t)|_{F}, \quad{ }^{\forall} \chi \in X^{*}(T)_{F} .
$$

(Note that $X^{*}(T) \otimes \mathbb{R} \simeq X^{*}(A) \otimes \mathbb{R}$.) 
B.2.2. Weyl group, Bruhat order and Bruhat decomposition. Let $\mathbf{W}=N_{G}(A) / Z_{G}(A)=N_{G}(A) / T$ be the relative Weyl group of $G$. We fix once and for all representatives $\bar{w} \in \mathbf{K}$. Then for any $w_{1}, w_{2} \in \mathbf{W}$, we have $\overline{w_{1} w_{2}}=t \overline{w_{1}} \overline{w_{2}}$ where $t \in T \cap \mathbf{K}$. As in 28, we shall denote unspecified element on $T \cap \mathbf{K}$ by $*$. Thus

$$
\overline{w_{1} w_{2}}=* \overline{w_{1}} \overline{w_{2}}
$$

where $*$ depends on $w_{1}$ and $w_{2}$ and also the choice of $\overline{w_{1}}$ and $\overline{w_{2}}$. We have the Bruhat decomposition

$$
G=\bigcup_{w \in \mathbf{W}} B w B
$$

Then for any $g \in G$, we may write $g=b \bar{w} n^{\prime}$ where $b \in B, w \in \mathbf{W}$ and $n^{\prime} \in N_{w}^{-}:=N \cap \bar{w}^{-1} \bar{N} \bar{w}$. In general, a choice of $\bar{w}$ is not unique. When we have $g \in N t \bar{w} N$ with $t \in T$ and $w \in \mathbf{W}$, then we write $\mathbf{w}(g)=w$ and denote by $a(g)=t$.

Let

$$
\mathcal{C}_{+}^{*}=\left\{\sum_{\alpha \in \Delta_{0}} c_{\alpha} \alpha: c_{\alpha} \in \mathbb{Z}_{\geq 0}\right\} .
$$

We write $\leq$ for the Bruhat order on $\mathbf{W}$. If $w_{1} \leq w_{2} \in \mathbf{W}$, then for any dominant $\lambda \in \mathfrak{a}_{0}^{*}$, we have

$$
w_{1} \lambda-w_{2} \lambda \in \mathcal{C}_{+}^{*} .
$$

For any $w, w^{\prime} \in \mathbf{W}$, we have

$$
B w B w^{\prime} B \subset \bigcup_{w^{\prime \prime} \leq w^{\prime}} B w w^{\prime \prime} B
$$

(cf. [28, (12)]).

Let $S(w)$ be the set of simple roots which appear in a reduced decomposition of $w$. (This set does not depend on the reduced decomposition). It is the smallest set $S \subset \triangle_{\circ}$ such that $w$ belongs to the group generated by $\left\{s_{\alpha}: \alpha \in S\right\}$. Alternatively,

$$
S(w)=\left\{\alpha \in \triangle_{\circ}: w \alpha^{*} \neq \alpha^{*}\right\}
$$

If $w_{1} \leq w_{2}$, then $S\left(w_{1}\right) \subset S\left(w_{2}\right)$. Let $S^{\circ}(w)=S\left(w w_{0}\right)$. Thus $S^{\circ}(w)=$ if and only if $w=w_{0}$ while $S^{\circ}(1)=\triangle_{0}$. Also, $S^{\circ}\left(w_{1}\right) \supset S^{\circ}\left(w_{2}\right)$ if $w_{1} \leq w_{2}$.

For any $S \subset \triangle_{0}$, let $\Phi_{\text {rel }}(S)$ be the set of roots in $\Phi_{\text {rel }}$ which are linear combinations of roots in $S$. This is a root subsystem of $\Phi_{\text {rel }}$ which corresponds to the standard Levi subgroup determined by $S$. We have

$$
\Phi_{\text {rel }}(S)=\left\{\beta \in \Phi:\left\langle\alpha^{*}, \beta^{\vee}\right\rangle=0 \text { for all } \alpha \notin S\right\} .
$$

Note that for any $w \in \mathbf{W}, w_{0} S\left(w_{0} w\right)=-S^{\circ}(w)$ and hence

$$
w_{0} \Phi_{\text {rel }}\left(S\left(w_{0} w\right)\right)=\Phi_{\text {rel }}\left(S^{\circ}(w)\right) .
$$

For $\beta \in \Phi_{\text {rel }}$, we denote by $s_{\beta} \in \mathbf{W}$ the corresponding reflection.

B.2.3. Spaces of Whittaker functions. Let $\Omega\left(N \backslash G, \psi_{N}\right)$ denote the space of Whittaker functions on $G$. It is well-known that for any normal open subgroup $K$ of $\mathbf{K}$, we have

$$
\sup \left|\alpha_{0}\right|(\operatorname{supp}(W)) \ll_{K} 1,
$$

for all $W \in \Omega\left(N \backslash G, \psi_{N}\right)^{K}$ and $\alpha \in \triangle_{0}$ with an extension to $\alpha_{0}$, that is the image of $\left|\alpha_{0}\right|$ on suppW is bounded above in terms of $K$ only. As in [28, following [5, Definition 5.1], we consider the space $\Omega^{\circ}\left(N \backslash G, \psi_{N}\right)$ consisting of those $W \in \Omega\left(N \backslash G, \psi_{N}\right)$ such that, for any $w \in \mathbb{W}$ and $\alpha \in S^{\circ}(w)$, we have $\inf \left|\alpha_{0}\right|\left(\operatorname{supp}_{B w B} W\right)>0$. Here, $\alpha_{0}$ is an extension of $\alpha$ to $T$.

For $\alpha \in \Phi_{\text {rel }}$, we denote by $\Phi_{\alpha}$ the subset of $\Phi$ consisting of roots whose restriction to $A$ is $\alpha$. Note that for any $\alpha \in \Phi_{\text {rel }}$, there is an extension $\beta \in \Phi$, however this is not unique in general.

For any $w \in \mathbf{W}$ and $\varepsilon>0$, let

$$
A^{\varepsilon}(w)=\left\{t \in T:\left|\beta^{*}\right|(t) \geq \varepsilon \text { for all } \beta \in \Phi_{\alpha} \text { with } \alpha \notin S^{\circ}(w)\right\} .
$$

Also, let

$$
B^{\varepsilon}(w)=\left\{t \in T:\left|\beta^{*}\right|(t) \leq 1 / \varepsilon \text { for all } \beta \in \Phi_{\alpha} \text { with } \alpha \in S(w),\left|\beta^{*}\right|(t) \geq \varepsilon \text { for all } \beta \in \Phi_{\alpha} \text { with } \alpha \notin S^{\circ}(w)\right\} .
$$

Further, we set $A_{s}^{\varepsilon}(w)=A^{\varepsilon}(w) \cap A$ and $B_{s}^{\varepsilon}(w)=B^{\varepsilon}(w) \cap A$.

We say that a set $C \subset G$ is bounded modulo $N$ if there exists a compact set $D \subset G$ such that $C \subset N D$. Denote by $\Omega^{\#}\left(N \backslash G, \psi_{N}\right)$ the subspace of those $W \in \Omega\left(N \backslash G, \psi_{N}\right)$ such that for any $w \in \mathbf{W}$ and $\varepsilon>0, \operatorname{supp}_{N A^{\varepsilon}(w) w N} W$ is bounded modulo $N$. 
B.2.4. The main technical statements. Theorem B.1, B.2 readily follows from the following technical results.

Proposition B.1 (cf. Proposition 1 in [28] and Theorem 9.5 in [5]). We have $\Omega^{\circ}\left(N \backslash G, \psi_{N}\right) \subset \Omega^{\#}\left(N \backslash G, \psi_{N}\right)$.

Lemma B.1 (cf. Lemma 1 in [28]). If $W \in \Omega^{\#}\left(N \backslash G, \psi_{N}\right)$, then for any $w \in \mathbb{W}$ and $\varepsilon>0$, the function $(t, n) \mapsto W(t \bar{w} n)$, $(t, n) \in B^{\varepsilon}(w) \times N_{w}^{-}$is compactly supported.

Proposition B.2 (cf. Proposition 2 in [28] and Theorem 7.3 in [5]). For any normal open subgroup K of $\mathbf{K}$, there exists a compact open subgroup $N^{\prime}$ of $N$ such that $R_{N^{\prime}, \psi_{N}} W \in \Omega^{\circ}\left(N \backslash G, \psi_{N}\right)$ for any $W \in \Omega\left(N \backslash G, \psi_{N}\right)^{K}$

B.2.5. A lemma on Bruhat orders. Suppose that $\alpha \in \triangle_{0}$ is of type (I). We may choose $n_{ \pm \alpha}$ and $\overline{s_{\alpha}}$ and so that

$$
n_{\alpha}(x) n_{-\alpha}\left(-x^{-1}\right)=\alpha^{\vee}(x) \overline{s_{\alpha}} n_{\alpha}(-x)
$$

and

$$
{\overline{s_{\alpha}}}^{-1} n_{\alpha}(x) \overline{s_{\alpha}}=n_{-\alpha}(-x)=n_{\alpha}\left(-x^{-1}\right) \alpha^{\vee}\left(x^{-1}\right) \overline{s_{\alpha}} n_{\alpha}\left(-x^{-1}\right), \quad x \in F_{\alpha}^{\times} .
$$

On the other hand, suppose that $\alpha$ is of type (II). Then we may take $\overline{s_{\alpha}}$ so that

$$
x_{\alpha}(r, m) x_{-\alpha}\left(-r m^{-1}, \bar{m}^{-1}\right)=\alpha^{\vee}(m) \overline{s_{\alpha}} x_{\alpha}(-(r \bar{m}) / m, \bar{m})
$$

and

$$
\overline{s_{\alpha}} x_{\alpha}(r, m){\overline{s_{\alpha}}}^{-1}=x_{-\alpha}(-r, m)=x_{\alpha}\left(-r \bar{m}^{-1}, m^{-1}\right) \alpha^{\vee}\left(\bar{m}^{-1}\right) \overline{s_{\alpha}} x_{\alpha}\left(-r \bar{m}^{-1}, \bar{m}^{-1}\right) .
$$

for any $r, m \in E_{\alpha}$ such that $N_{E_{\alpha} / F_{\alpha}}(r)=-\operatorname{Tr}_{E_{\alpha} / F_{\alpha}}(m)$.

Lemma B.2. Let $w, w^{\prime} \in W$ and $\alpha \in \Phi_{\mathrm{rel},+}$. Assume that $w^{\prime} s_{\alpha} \leq w$ and $w \alpha \in \Phi_{\mathrm{rel},+}$. Then $w \alpha \in \Phi\left(S\left(w w^{\prime-1}\right)\right)$.

Proof. This is just a restatement of [28, Lemma 2] for $\Phi_{\text {rel }}$.

Lemma B.3. Let $K$ be a compact open normal subgroup of $\mathbf{K}$. Then for any $g \in G$ and $\alpha \in \triangle_{0}$, either

(1) there exists $k \in K$ such that $\mathbf{w}\left(g \overline{s_{\alpha}} k\right)=\mathbf{w}(g) s_{\alpha}$ and $H\left(a\left(g \overline{s_{\alpha}} k\right)\right)=H(a(g))$, or

(2) $\mathbf{w}(g) \alpha \in \Phi_{\mathrm{rel},-}, \mathbf{w}\left(g \overline{s_{\alpha}}\right)=\mathbf{w}(g)$ and $H\left(a\left(g \overline{s_{\alpha}}\right)\right)-H(a(g))=x\left(\mathbf{w}(w) \cdot\left(\alpha^{\vee}\right)\right)$ with $x \ll_{K} 1$.

Proof. We follow the proof of [28, Lemma 3]. Indeed, when $\alpha$ is of type (I), our lemma is proved in the same way as their lemma. Now, suppose that $\alpha$ is of type (II). Further, we may suppose that $g=\bar{w} n$ where $w=\mathbf{w}(g) \in \mathbf{W}$ and $n \in N_{w}^{-}$.

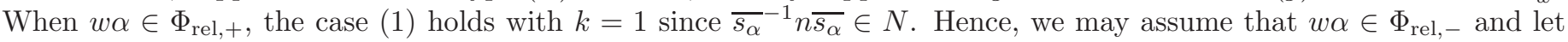
$w^{\prime}=w s_{\alpha}$ so that $w^{\prime} \alpha \in \Phi_{\mathrm{rel},+}$.

Write $n=n_{1} x_{\alpha}(r, m)=x_{\alpha}(r, m) n_{2}$ where $n_{1}, n_{2} \in N^{\alpha}$ and $r, m \in E_{\alpha}$ such that $N_{E_{\alpha} / F_{\alpha}}(r)=-\operatorname{Tr}_{E_{\alpha} / F_{\alpha}}(m)$. Suppose that $|m|$ is so small that $x_{\alpha}(r, m) \in K$. Then $k:={\overline{s_{\alpha}}}^{-1} x_{\alpha}(-r, \bar{m}) \overline{s_{\alpha}} \in \mathbf{K}$, and we have

$$
g \overline{s_{\alpha}} k=\bar{w} n_{1} x_{\alpha}(r, m) \overline{s_{\alpha}} k=\bar{w} n_{1} \overline{s_{\alpha}}=* \overline{w^{\prime}} \overline{s_{\alpha}}-1 n_{1} \overline{s_{\alpha}} \in * \overline{w^{\prime}} N
$$

Otherwise, we write

$$
g \overline{s_{\alpha}}=\bar{w} x_{\alpha}(r, m) n_{2} \overline{s_{\alpha}}=\bar{w} x_{\alpha}(r, m) \overline{s_{\alpha}} n_{3}
$$

where $n_{3}={\overline{s_{\alpha}}}^{-1} n_{2} \overline{s_{\alpha}} \in N$. From (B.5), we have

$$
\begin{aligned}
g \overline{s_{\alpha}}=* \overline{w^{\prime}} \bar{s}_{\alpha}^{-1} x_{\alpha}(r, m) \overline{s_{\alpha}} n_{3} & =* \overline{w^{\prime}} x_{\alpha}\left(-r \bar{m}^{-1}, m^{-1}\right) \alpha^{\vee}\left(\bar{m}^{-1}\right) \overline{s_{\alpha}} x_{\alpha}\left(-r \bar{m}^{-1}, \bar{m}^{-1}\right) n_{3} \\
& =*\left(\overline{w^{\prime}} x_{\alpha}\left(-r \bar{m}^{-1}, m^{-1}\right) \bar{w}^{\prime}-1\right)\left(\overline{w^{\prime}} \alpha^{\vee}\left(\bar{m}^{-1}\right) \bar{w}^{-1}\right) \bar{w} x_{\alpha}\left(-r \bar{m}^{-1}, \bar{m}^{-1}\right)
\end{aligned}
$$

Since $w^{\prime} \alpha \in \Phi_{\text {rel, }+},\left(\overline{w^{\prime}} x_{\alpha}\left(-r \bar{m}^{-1}, m^{-1}\right) \bar{w}^{\prime}-1\right) \in N$. Further, we know

$$
\overline{w^{\prime}} \alpha^{\vee}\left(\bar{m}^{-1}\right) \bar{w}^{-1}=\bar{w} \cdot\left(\alpha^{\vee}(m)\right)
$$

Thus, we have

$$
H\left(a\left(g \overline{s_{\alpha}}\right)\right)=x\left(\mathbf{w}(w) \cdot\left(\alpha^{\vee}\right)\right)
$$

with $x \ll_{K} 1$.

For any subset $S \subset \triangle_{0}$ and $X>0$, we define

$$
\mathcal{C}(S)_{\geq-X}=\left\{\sum_{\alpha \in S} c_{\alpha} \alpha^{\vee}: c_{\alpha} \geq-X \quad{ }^{\forall} \alpha \in S\right\} .
$$

For $w \in \mathbf{W}$, we fix its reduced decomposition by $w=s_{\alpha_{k}} \cdots s_{\alpha_{1}}$ with $\alpha_{i} \in \triangle_{0}$. We shall use the following notational conventions, which implicitly depend on the reduced decomposition we chose (see the beginning of [28, 3.2]).

For any $i=1, \ldots, k$, let $w_{i}=s_{\alpha_{i-1}} \cdots s_{\alpha_{1}}$ and ${ }_{i} w=s_{\alpha_{k}} \cdots s_{\alpha_{i+1}}$ so that $w={ }_{i} w s_{\alpha_{i}} w_{i}$. We also write $\beta_{i}=w_{i}{ }^{-1} \alpha_{i}$ so that $\left\{\beta_{1}, \ldots, \beta_{k}\right\}=\left\{\beta \in \Phi_{\mathrm{rel},+}: w \beta \in \Phi_{\mathrm{rel},-}\right\}$. Note that $w \beta_{i}=-{ }_{i} w \alpha_{i} \in \Phi_{\mathrm{rel}}(S(w))$ for all $i$. 
We may also write $N_{w}^{-}={ }_{i} N N_{\beta_{i}} N_{i}$ where $N_{i}=N_{w_{i}}^{-}$and ${ }_{i} N=w_{i}^{-1} s_{\alpha_{i}}^{-1} N_{i}^{-} s_{\alpha_{i}} w_{i}=w_{i+1}^{-1} N_{i w}^{-} w_{i+1}$. Note that $N_{1}=$ ${ }_{k} N=1, N_{i+1}=N_{\beta_{i}} \ltimes N_{i}$ and ${ }_{i-1} N={ }_{i} N \rtimes N_{\beta_{i}}$. Let $n \in N_{w}^{-}$. For any $i$, we can write uniquely as

$$
n= \begin{cases}{ }_{i} n n_{\beta_{i}}\left(x_{i}\right) n_{i} & \text { if } \alpha_{i} \text { if of type (I) } \\ { }_{i} n n_{\beta_{i}}\left(r_{i}, m_{i}\right) n_{i} & \text { if } \alpha_{i} \text { if of type (II) }\end{cases}
$$

where ${ }_{i} n \in{ }_{i} N n_{i} \in N_{i}, x_{i} \in F_{\alpha_{i}}$ and $r_{i}, m_{i} \in E_{\alpha_{i}}$ such that $N_{E_{\alpha_{i}} / F_{\alpha_{i}}}\left(r_{i}\right)=-\operatorname{Tr}_{E_{\alpha_{i}} / F_{\alpha_{i}}}\left(m_{i}\right)$. We have

$$
n_{i+1}= \begin{cases}n_{\beta_{i}}\left(x_{i}\right) n_{i} & \text { if } \alpha_{i} \text { if of type (I) } \\ n_{\beta_{i}}\left(r_{i}, m_{i}\right) n_{i} & \text { if } \alpha_{i} \text { if of type (II) }\end{cases}
$$

Lemma B.4. Let $w={ }_{i} w s_{\alpha_{i}} w_{i}$ be as above and let $g={ }_{i} w n w_{i}$ with $n \in N$. Then $\mathbf{w}(g)<w$. Moreover, for any $K$ and any $n \in N$, there exists $k \in K$ such that

(1) $\mathbf{w}(g k)={ }_{i} w \tilde{w}_{i}$ for some $\tilde{w}_{i} \leq w_{i}$ (depending on $g$ ), and

(2) $H(a(g k)) \in \mathcal{C}\left(S^{\circ}(\mathbf{w}(g k))\right)_{\geq-X}$ with $X \ll_{K} 1$.

Proof. This lemma is proved similarly as [28, Lemma 4] using Lemma B.3] instead of [28, Lemma 3]

Lemma B.5. Let $w$ be as before and suppose that $\alpha_{i}$ is of type (II). Let $g=\bar{w} n w_{i}^{-1} x_{\alpha_{i}}(r, m) w_{i}$ with $n \in{ }_{i} N$ and $r, m \in E_{\alpha_{i}}$ such that $N_{E_{\alpha_{i}} / F_{\alpha_{i}}}(r)=-\operatorname{Tr}_{E_{\alpha_{i}} / F_{\alpha_{i}}}(m)$. that

Then $\mathbf{w}\left(g w_{i}^{-1} x_{-\alpha_{i}}\left(-r m^{-1}, \bar{m}^{-1}\right) w_{i}\right)<w=\mathbf{w}(g)$. Moreover, given $K$, either $|m| \ll_{K} 1$ or there exists $k \in K$ such

(1) $\mathbf{w}(g k)<w=\mathbf{w}(g)$

(2) $H(a(g k))-\nu(m) w w_{i}^{-1} \cdot\left(\alpha_{i}^{\vee}\right) \in \mathcal{C}\left(S^{\circ}(\mathbf{w}(g k))\right)_{-X}$ with $X \ll_{K} 1$, and

(3) $w w_{i}^{-1} \alpha_{i} \in \Phi_{\text {rel }}\left(S^{\circ}(\mathbf{w}(g k))\right)$.

Proof. Let $k:=w_{i}^{-1} x_{-\alpha_{i}}\left(-r m^{-1}, \bar{m}^{-1}\right) w_{i}$. If $|m|$ is sufficiently large with respect to $K$, then $k \in K$. By (B.4), we have

$$
g k=\bar{w} n w_{i}^{-1} \alpha_{i}^{\vee}(m) \overline{s_{i}} x_{\alpha_{i}}(-(r \bar{m}) / m, \bar{m}) w_{i},
$$

which we can write as

$$
\left(\bar{w} w_{i}^{-1} \alpha_{i}^{\vee}(m) w_{i} \bar{w}^{-1}\right) \bar{w} n^{\prime} w_{i}^{-1} \overline{s_{\alpha_{i}}} x_{\alpha_{i}}(-(r \bar{m}) / m, \bar{m}) w_{i}
$$

where $n^{\prime}=\left(w_{i}^{-1} \alpha_{i}^{\vee}(m) w_{i}\right)^{-1} n w_{i}^{-1} \alpha_{i}^{\vee}(m) w_{i}$. Changing $n^{\prime}$ by a conjugate of it by an element of $T \cap \mathbf{K}$, if necessary, we can write this as

$$
*\left(\bar{w} w_{i}^{-1} \alpha_{i}^{\vee}(m) w_{i} \bar{w}^{-1}\right) \bar{w} n^{\prime}{\overline{s_{\alpha_{i}} w_{i}}}^{-1} x_{\alpha_{i}}(-(r \bar{m}) / m, \bar{m}) w_{i}=*\left(\bar{w} w_{i}^{-1} \alpha_{i}^{\vee}(m) w_{i} \bar{w}^{-1}\right) \bar{i} \bar{w} n^{\prime \prime} x_{\alpha_{i}}(-(r \bar{m}) / m, \bar{m}) w_{i}
$$

where $n^{\prime \prime}={\overline{s_{\alpha_{i}} w_{i}}}^{\prime}{\overline{s_{\alpha_{i}} w_{i}}}^{-1} \in N_{i}^{-}$. Then we conclude that

$$
g k \in *\left(\bar{w} w_{i}^{-1} \alpha_{i}^{\vee}(m) w_{i} \bar{w}^{-1}\right) \bar{i} \bar{w} N \bar{w}_{i} .
$$

All but the last part directly follows from Lemma B.4. Further, the last part follows from Lemma B.2 as in the proof of [28, Lemma 5].

Similarly, when $\alpha_{i}$ is of type (I), we have the following lemma, which is proved in the same way as the proof of [28, Lemma 5].

Lemma B.6. Let $w$ be as before and suppose that $\alpha_{i}$ is of type (I). Let $g=\bar{w} n w_{i}^{-1} n_{\alpha_{i}}(x) w_{i}$ with $n \in{ }_{i} N$ and $x \in F_{\alpha_{i}}$.

Then $\mathbf{w}\left(g w_{i}^{-1} x_{-\alpha_{i}}\left(-x^{-1}\right) w_{i}\right)<w=\mathbf{w}(g)$. Moreover, given $K$, either $|x| \ll_{K} 1$ or there exists $k \in K$ such that

(1) $\mathbf{w}(g k)<w=\mathbf{w}(g)$

(2) $H(a(g k))-\nu(m) w w_{i}^{-1} \cdot\left(\alpha_{i}^{\vee}\right) \in \mathcal{C}\left(S^{\circ}(\mathbf{w}(g k))\right)_{-X}$ with $X \ll_{K} 1$, and

(3) $w w_{i}^{-1} \alpha_{i} \in \Phi_{\text {rel }}\left(S^{\circ}(\mathbf{w}(g k))\right)$.

Lemma B.7. Let $w \in \mathbf{W}$ and let $\Phi_{w}=\left\{\beta \in \Phi_{\mathrm{rel},+}: w^{-1} \beta<0\right\}$. Then for any $n \in N$ we have $H(\bar{w} n)=\sum_{\alpha \in \Phi_{w}} c_{\alpha} \alpha^{\vee}$ with $c_{\alpha} \leq 0$, for all $\alpha \in \Phi_{w}$. Thus, $\left|\alpha^{*}\right|(a(\bar{w})) \leq 1$ for all $\alpha \in \triangle_{0}$ with equality if $\alpha \notin S(w)$. Moreover, the map $n \mapsto H(\bar{w} n)$ from $N_{w}^{-}$to $\mathfrak{a}_{T}$ is proper.

Proof. This lemma is proved in the same way as [28, Lemma 6] using the relation

$$
H\left(\overline{s_{\alpha}} x_{\alpha}(r, m)\right)=\min (0,-v(y)) \alpha^{\vee}
$$

for any $\alpha \in \triangle_{0}$ of type (I) (resp. type (II)) because of (B.3) (resp. (B.5)).

The following lemma is proved in the same way as [28, Lemma 7], which is a special case of Proposition B.1.

Lemma B.8. For any $W \in \Omega^{\circ}\left(N \backslash G, \psi_{N}\right), w \in \mathbf{W}$ and $\varepsilon>0$, $\operatorname{supp}_{A^{\varepsilon}(w) w} W$ is compact. 
B.3. Proof of of Proposition B.1. We follow the argument in [28, 3.4] (see also the proof of [5, Theorem 9.5]).

We prove by induction on $\ell(w)$ that for any $w \in \mathbf{W}$ and $\varepsilon>0$

$$
\text { for any } W \in \Omega^{\circ}\left(N \backslash G, \psi_{N}\right), \operatorname{supp}_{A^{\varepsilon}(w) w N} W \text { is bounded modulo } N \text {. }
$$

For $w=1$, this is a special case of Lemma B.8 above. Assume now (B.7) holds for all $w^{\prime}$ with $\ell\left(w^{\prime}\right)<\ell(w)$.

Let $w=s_{\alpha_{k}} \cdots s_{\alpha_{1}}$ be a reduced decomposition of $w$ and let $\beta_{1}, \ldots, \beta_{k},{ }_{i} w, w_{i}, N_{i},{ }_{i} N$ be as above. Let $n \in N_{w}^{-}$and write

$$
n= \begin{cases}{ }_{i} n n_{\beta_{i}}(x) n_{i} & \text { if } \alpha_{i} \text { if of type (I) } \\ { }_{i} n n_{\beta_{i}}(r, m) n_{i} & \text { if } \alpha_{i} \text { if of type (II) }\end{cases}
$$

To prove (B.7), we will show by induction on $i$ that

$$
\text { for any } W \in \Omega^{\circ}\left(N \backslash G, \psi_{N}\right) \text {, } \operatorname{supp}_{N A^{\varepsilon}(w)_{i} N} W \text { is bounded modulo } N \text {. }
$$

For $i=k$ this follows from Lemma B.8 since ${ }_{k} N=1$. For the induction step, assume that (B.8) holds for $i$ and we will show it for $i-1$.

Let $W \in \Omega^{\circ}\left(N \backslash G, \psi_{N}\right)^{K}$. Suppose that $\alpha_{i}$ is of type (I), and assume that $W(g) \neq 0$ with $g=t \bar{w} n n_{\beta_{i}}(x), n \in{ }_{i} N$ and $t \in A^{\varepsilon}(w)$. Then we may apply the same argument as the proof of [28, Proposition 1] by Lemma B.6] and thus (B.8) holds for $i-1$. Now, suppose that $\alpha_{i}$ is of type (II), and assume that $W(g) \neq 0$ with $g=t \bar{w} n w_{i}^{-1} n_{\alpha_{i}}(r, m) w_{i}, n \in{ }_{i} N$ and $t \in A^{\varepsilon}(w)$.

By LemmaB.5, either $|m| \ll W 1$ or there exists $k \in K$ such that $w^{\prime}:=\mathbf{w}(g k)<w, w \beta_{i} \in \Phi_{\text {rel }}\left(S^{\circ}\left(w^{\prime}\right)\right)$ and if $t^{\prime}=a(g k)$, then $H\left(t^{\prime}\right)-H(t)-\nu(m) w w_{i}^{-1} \cdot\left(\alpha_{i}^{\vee}\right) \in \mathcal{C}\left(S^{\circ}\left(w^{\prime}\right)\right)_{-T}$ with $T \ll_{K} 1$.

In the former case, we can use the induction hypothesis of $(\mathbb{B} .8)$ for the finitely may translates $R\left(n^{\prime}\right) W$ where $n^{\prime}$ lies in a suitable compact subgroup of ${\overline{w_{i}}}^{-1} N_{\alpha_{i}} \overline{w_{i}}$ (depending only on $W$ ).

In the latter case,

$$
\left|\alpha^{*}\right|\left(t^{\prime}\right)=\left|\alpha^{*}\right|(t)
$$

for all $\alpha \notin S^{\circ}\left(w^{\prime}\right)$. Hence, $t^{\prime} \in A^{\varepsilon}\left(w^{\prime}\right)$ since $t \in A^{\varepsilon}(w)$. Since $w^{\prime}<w$, we may apply the inductive assumption, namely (B.7) for $w^{\prime}$. Then we find that $g k$ and therefore, $g$ is compactly supported modulo $N$, which finishes the proof of Proposition B.1

B.4. Proof of Lemma B.1. This is proved in the same way as the proof of [28, Lemma 1] using Lemma B.7 instead of [28, Lemma 6].

B.5. Proof of Proposition 2. Let $U_{0}=N \cap \mathbf{K}$. Fix $a \in A$ such that $|\alpha(a)|>1$, for all $\alpha \in \triangle_{0}$ (and hence for all $\left.\alpha \in \Phi_{\mathrm{rel},+}\right)$. For any $m \geq 1$ define $U_{m}=a^{m} U_{0} a^{-m}$. Thus, $U_{1} \subset U_{2} \subset \cdots$ and $\cup_{m=1}^{\infty} U_{m}=N$. Set

$$
W_{m}:=R_{U_{m}, \psi_{N}} W=\frac{1}{\operatorname{vol}\left(U_{m}\right)} \int_{U_{m}} R(u) W \psi_{N}(u)^{-1} d u \in \Omega\left(N \backslash G, \psi_{N}\right) .
$$

Clearly,

$$
W_{m}(g u)=\psi_{N}(u) W_{m}(g), \quad \text { for all } u \in U_{m}, g \in G .
$$

We fix the reduced decomposition $s_{\tilde{\alpha}_{l}} \cdots s_{\tilde{\alpha}_{1}}$ of $w_{0}$. Note that $N_{w_{0}}^{-}=N$ Set

$$
\tilde{\beta}_{i}=\tilde{w}_{i}^{-1} \tilde{\alpha}_{i} .
$$

If necessary, after renumbering, we may suppose that $\tilde{\alpha}_{1}, \ldots, \tilde{\alpha}_{s}$ are of type (I) and $\tilde{\alpha}_{s+1}, \ldots, \tilde{\alpha}_{l}$ are ot type (II).

Lemma B.9 (cf. Lemma 8 in [28]). Let $n=n_{\tilde{\beta}_{l}}\left(x_{l}\right) \cdots n_{\tilde{\beta}_{m}}\left(x_{m}\right) \cdot x_{\beta_{m+1}}\left(p_{m+1}, q_{m+1}\right) \cdots x_{\beta_{1}}\left(p_{1}, q_{1}\right)$. Then $u \in U_{m}$ if and only if $\left|x_{i}\right| \leq\left|\tilde{\beta}_{i}\left(a^{m}\right)\right|$ and $\left|q_{j}\right| \leq\left|\tilde{\beta}_{i}\left(a^{2 m}\right)\right|$ for all $i, j$.

Proof. Clearly, we have

$$
a^{m}\left(N_{\tilde{\beta}_{i}} \cap \mathbf{K}\right) a^{-m}=n_{\tilde{\beta}_{i}}\left(\tilde{\beta}_{i}\left(a^{m}\right) \mathcal{O}\right)
$$

for $1 \leq i \leq s$, and

$$
a^{m}\left(N_{\tilde{\beta}_{i}} \cap \mathbf{K}\right) a^{-m}=\left\{n_{\tilde{\beta}_{i}}\left(\tilde{\beta}_{i}\left(a^{m}\right) p, \tilde{\beta}_{i}\left(a^{2 m}\right) q\right): p, q \in \mathcal{O}_{E_{\alpha_{i}}}, N_{E_{\alpha_{i}} / E_{\alpha_{i}}}(p)=-\operatorname{Tr}_{E_{\alpha_{i}} / F_{\alpha_{i}}}(q)\right\}
$$

for $s+1 \leq i \leq l$. If $\tilde{\beta}_{i}\left(a^{2 m}\right) q \in \mathcal{O}_{E_{\alpha_{i}}}$, then $\tilde{\beta}_{i}\left(a^{m}\right) p \in \mathcal{O}_{E_{\alpha_{i}}}$ because of the relation $N_{E_{\alpha_{i}} / F_{\alpha_{i}}}(p)=-\operatorname{Tr}_{E_{\alpha_{i}} / F_{\alpha_{i}}}(q)$. Then the "if" direction follows.

Let prove the "only if" direction for $j$. For the induction step, as well as for the base of the induction, we may assume that $p_{1}=q_{1}=\cdots=p_{j-1}=q_{j-1}=0$, i.e. $n \in{ }_{i-1} \tilde{N}$. In this case, we observe that

$$
x_{\beta_{j}}\left(p_{j}, q_{j}\right)=\overline{\tilde{w}}_{i}^{-1} \pi_{\tilde{\alpha}_{i}}\left(\widetilde{\tilde{w}}_{i} n \widetilde{w}_{i}^{-1}\right) \widetilde{w}_{i},
$$


where $\pi_{\tilde{\alpha}_{i}}: N \rightarrow N_{\tilde{\alpha}_{i}}$ is the canonical projection. Write $n=a^{m} n^{\prime} a^{-m}$ where $n^{\prime} \in{ }_{j-1} \tilde{N} \cap \mathbf{K}$. Then since $\pi_{\tilde{\alpha}_{i}}$ is equivariant with respect to conjugation by $A$ we get

$$
x_{\beta_{j}}\left(p_{j}, q_{j}\right)={\overline{\tilde{w}_{i}}}^{-1} \pi_{\tilde{\alpha}_{i}}\left(\overline{\tilde{w}}_{i} a^{m} n^{\prime} a^{-m}{\overline{\tilde{w}_{i}}}^{-1}\right) \overline{\tilde{w}_{i}}=a^{m}{\overline{\tilde{w}_{i}}}^{-1} \pi_{\tilde{\alpha}_{i}}\left(\bar{w}_{i} n^{\prime} \bar{w}_{i}^{-1}\right) \overline{\tilde{w}_{i}} a^{-m},
$$

or,

$$
x_{\beta_{j}}\left(\tilde{\beta}_{i}\left(a^{-m}\right) p_{j}, \tilde{\beta}_{i}\left(a^{-2 m}\right) q_{j}\right)={\overline{\tilde{w}_{i}}}^{-1} \pi_{\tilde{\alpha}_{i}}\left({\overline{\tilde{w}_{i}}}^{\prime}{\overline{\tilde{w}_{i}}}^{-1}\right) \overline{\tilde{w}_{i}} \in \mathbf{K}
$$

Then the required inequality follows.

Lemma B.10 (cf. Lemma 9 in [28]). Let $n={ }_{i} n x_{\tilde{\beta}_{i}}\left(p_{i}, q_{i}\right)$ with ${ }_{i} n \in{ }_{i} \tilde{N}$ and $p_{i}, q_{i} \in E_{\alpha_{i}}$ such that $N_{E_{\alpha_{i}} / F_{\alpha_{i}}}\left(p_{i}\right)=$ $-\operatorname{Tr}_{E_{\alpha_{i}} / F_{\alpha_{i}}}\left(q_{i}\right)$. Assume that $x_{\tilde{\beta}_{i}}\left(p_{i}, q_{i}\right) \notin U_{m}$. Then for any $n^{\prime} \in U_{m}$, we have $n n^{\prime}={ }_{i} \tilde{n} x_{\tilde{\beta}_{i}}\left(\tilde{p}_{i}, \tilde{q}_{i}\right) \tilde{n}_{i}$ where ${ }_{i} \tilde{n} \in{ }_{i} \tilde{N}$ $\tilde{n}_{i} \in U_{m} \cap \tilde{N}_{i}$ and $\left|q_{i}\right|=\left|\tilde{q}_{i}\right|$.

Proof. Write $n^{\prime}={ }_{i} n^{\prime} x_{\tilde{\beta}_{i}}\left(p_{i}^{\prime}, q_{i}^{\prime}\right) n_{i}^{\prime}$ with ${ }_{i} n^{\prime} \in{ }_{i} \tilde{N} p_{i}, q_{i} \in E$ such that $N_{E / L}\left(p_{i}\right)=-\operatorname{Tr}_{E / L}\left(q_{i}\right)$ and $n_{i}^{\prime} \in \tilde{N}_{i}$. Then

$$
n n^{\prime}={ }_{i} n x_{\tilde{\beta}_{i}}\left(p_{i}, q_{i}\right){ }_{i} n^{\prime} x_{\tilde{\beta}_{i}}\left(p_{i}^{\prime}, q_{i}^{\prime}\right) n_{i}^{\prime}={ }_{i} n n^{\prime \prime} x_{\tilde{\beta}_{i}}\left(p_{i}+p_{i}^{\prime}, q_{i}+q_{i}^{\prime}-p_{i} \overline{p_{i}^{\prime}}\right) n_{i}^{\prime},
$$

where $n^{\prime \prime}=x_{\tilde{\beta}_{i}}\left(p_{i}, q_{i}\right)_{i} n^{\prime} x_{\tilde{\beta}_{i}}\left(p_{i}, q_{i}\right)^{-1} \in{ }_{i} \tilde{N}$. From the definition of $N_{i}$, we may write $n_{i}=n_{\tilde{\beta}_{l(i)}}\left(x_{l(i)}\right) \cdots n_{\tilde{\beta}_{m(i)}}\left(x_{m(i)}\right)$. $x_{\beta_{m(i)+1}}\left(p_{m(i)+1}, q_{m(i)+1}\right) \cdots x_{\beta_{1}}\left(p_{1}, q_{1}\right)$. Since $n_{j+1}=n_{j} n_{\beta_{j}}$ or $n_{j+1}=n_{j} x_{\beta_{j}}$, we see that $\left|q_{1}\right| \leq\left|\beta_{i}\left(a^{2 m}\right)\right|$ by Lemma B.9. Repeatedly using the lemma, we see that $n_{i}^{\prime} \in U_{m}$. Further, we see that

$$
\left|q_{i}^{\prime}\right| \leq\left|\beta_{i}\left(a^{2 m}\right)\right| .
$$

Since $x_{\tilde{\beta}_{i}}\left(p_{i}, q_{i}\right) \notin U_{m}$, we have

$$
\left|\beta_{i}\left(a^{2 m}\right)\right|<\left|q_{i}\right|
$$

Then it is easy to see that

$$
\left|p_{i} \overline{p_{i}^{\prime}}\right|<\left|q_{i}\right|
$$

and thus

The lemma follows.

$$
\left|q_{i}+q_{i}^{\prime}-p_{i} \overline{p_{i}^{\prime}}\right|=\left|q_{i}\right| .
$$

Now let $w \in \mathbf{W}$ and set $N_{w}^{+}=N \cap \bar{w}^{-1} N \bar{w}$. Fix a reduced decomposition $s_{\alpha_{k}} \cdots s_{\alpha_{1}}$ of $w$ and use the notation of Section B.2.5.

Corollary B.1. Let $i=1, \ldots, k$ and $n={ }_{i} n n_{\beta_{i}}\left(p_{i}, q_{i}\right)$ with ${ }_{i} N$ and $p_{i}, q_{i} \in E_{\alpha_{i}}$ such that $N_{E_{\alpha_{i}} / F_{\alpha_{i}}}\left(p_{i}\right)=-\operatorname{Tr}_{E_{\alpha_{i}} / F_{\alpha_{i}}}\left(q_{i}\right)$. Assume that $n_{\beta_{i}}\left(p_{i}, q_{i}\right) \notin U_{m}$. Then for any $n^{\prime} \in U_{m}$, we have $n n^{\prime}={ }_{i} \tilde{n} n_{\tilde{\beta}_{i}}\left(\tilde{p}_{i}, \tilde{q}_{i}\right) \tilde{n}_{i}$ where ${ }_{i} \tilde{n} \in N_{w}^{+} \cdot{ }_{i} \tilde{N} \tilde{n}_{i} \in U_{m}$ and $\left|q_{i}\right|=\left|\tilde{q}_{i}\right|>\left|\beta_{i}\left(a^{2 m}\right)\right|$.

For the convenience to the reader, we record a similar result in the case of type (I) by Lapid-Mao [28.

Corollary B.2 (Corollary 1 in [28]). Let $i=1, \ldots, k$ and $n={ }_{i} n n_{\beta_{i}}\left(x_{i}\right)$ with ${ }_{i} N$. Assume that $n_{\beta_{i}}\left(x_{i}\right) \notin U_{m}$. Then for any $n^{\prime} \in U_{m}$, we have $n n^{\prime}={ }_{i} \tilde{n} n_{\tilde{\beta}_{i}}\left(\tilde{p}_{i}, \tilde{q}_{i}\right) \tilde{n}_{i}$ where ${ }_{i} \tilde{n} \in N_{w}^{+} \cdot{ }_{i} \tilde{N} \tilde{n}_{i} \in U_{m}$ and $\left|x_{i}\right|>\left|\beta_{i}\left(a^{m}\right)\right|$.

\section{B.5.1. A special case.}

Lemma B.11. There exists $M$ such that for all $W \in \Omega\left(N \backslash G, \psi_{N}\right), w \in \mathbf{W} m \geq M$ and $\alpha \in S^{\circ}(w)$ we have $\inf \left|\alpha_{0}\right|\left(\operatorname{supp}_{B w} W_{m}\right)>0$. Here, $\alpha_{0}$ is an extension of $\alpha$ to $T$

Proof. Suppose that $\alpha \in \triangle_{0}$ is of type (I) (resp. type (II)). Then we let $\psi_{\alpha}: F_{\alpha} \rightarrow \mathbb{C}$ (resp. $\psi_{\alpha}: E_{\alpha} \rightarrow \mathbb{C}$ ) be the nontrivial character defined by

$$
\psi\left(n_{\alpha}(x)\right) \quad\left(\operatorname{resp} \cdot \psi\left(x_{\alpha}\left(x,-\frac{N_{E_{\alpha}} / F_{\alpha}(x)}{2}\right)\right)\right)
$$

Denote by $\operatorname{cond}\left(\psi_{\alpha}\right)$ its conductor, namely the maximal fractional ideal of $\mathcal{O}_{F_{\alpha}}$ or $\mathcal{O}_{E_{\alpha}}$ on which $\psi_{\alpha}$ is trivial. For any $\alpha, \beta \in \triangle_{0}$ of the same type, let $c_{\alpha, \beta} \in F_{\alpha}^{\times}$or $E_{\alpha}^{\times}$(depending on the type) such that $\psi_{\beta}=\psi_{\alpha}\left(c_{\alpha, \beta} \cdot\right)$.

Suppose that $\beta \in \triangle_{0}$ is of type (II) and there exists $\alpha \in \Phi_{\mathrm{rel},+} \backslash \triangle_{0}$ such that $\beta=w^{-1} \alpha$. Then we have

$$
\psi_{\beta}(x) W_{m}(t \bar{w})=W_{m}\left(t \bar{w} x_{\beta}\left(x,-\frac{N_{E_{\alpha} / F_{\alpha}}(x)}{2}\right)\right)=W_{m}\left(x_{\alpha}\left(* \alpha_{0}(t) x,-\frac{N_{E_{\alpha} / F_{\alpha}}\left(* \alpha_{0}(t) x\right)}{2}\right) t \bar{w}\right)=W_{m}(t \bar{w})
$$

for any $t \in T$ and all $x \in \beta\left(a^{m}\right) \mathcal{O}_{E_{\alpha}}$. It follows that in fact $W_{m}(s \bar{w})=0$ for all $s \in T$ provided that $\beta\left(a^{m}\right) \notin \operatorname{cond}\left(\psi_{\alpha}\right)$. If $\beta \in \triangle_{0}$ is of type (I) and there exists $\alpha \in \Phi_{\mathrm{rel},+} \backslash \triangle_{0}$ such that $\beta=w^{-1} \alpha$, then we can prove our claim in a similar way as above or as the proof of [28, Lemma 10]. 
On the other hand, suppose that these does not exist $\beta \in \triangle_{0}$ such that $\beta=w^{-1} \alpha$ for some $\alpha \in \Phi_{\text {rel, }+} \backslash \triangle_{0}$. Then using Steinberg [51, Lemma 89], in a similar way as [28, Lemma 10], we find that if $W_{m}(s \bar{w}) \neq 0$ and $m$ is sufficiently large, then

which concludes our proof.

$$
\left|\alpha_{0}\right|(s)=\left|c_{\alpha, w^{-1} \alpha}\right|
$$

B.5.2. The general case. To prove Proposition B.2, we will show by induction on $\ell(w)$ that, for any $w \in \mathbf{W}$,

$$
\begin{array}{r}
\text { there exists } M \text { depending on } K \text { such that for any } W \in \Omega\left(N \backslash G, \psi_{N}\right)^{K}, m \geq M \\
\text { and } \alpha \in S^{\circ}(w) \text { we have } \inf \left|\alpha_{0}\right|\left(\operatorname{supp}_{B w B} W_{m}\right)>0 .
\end{array}
$$

The case $w=1$ follows from Lemma B.11. To carry out the induction step assume that (B.10) holds for all $w^{\prime}<w$.

Fix a reduced decomposition of $w$ and use the notation of Section B.2.5. For any $m$ and $i=1, \ldots, k$, let

$$
\mathcal{B}_{w}(i, m)=\left\{b \bar{w} n: b \in B, n \in N_{w}^{-}, n_{i} \in U_{m}, n_{i+1} \notin U_{m}\right\} .
$$

Consider the following auxiliary statement.

There exists $M$ depending on $K$ such that for any $W \in \Omega\left(N \backslash G, \psi_{N}\right)^{K}, m \geq M$ and $\alpha \in S^{\circ}(w)$ we have $\inf \left|\alpha_{0}\right|\left(\operatorname{supp}_{\mathcal{B}_{w}(i, m)} W_{m}\right)>0$.

We will show this statement by induction on $i$. This will yield (B.10) for $w$. Indeed, we may take $M$ for which (B.11) holds for all $i$. Then, for any $m \geq M$ we have

$$
\inf \left|\alpha_{0}\right|\left(\operatorname{supp}_{\cup_{i=1}^{k} \mathcal{B}_{w}(i, m)} W_{m}\right)>0
$$

On the other hand, the complement of $\cup_{i=1}^{k} \mathcal{B}_{w}(i, m)$ in $\mathcal{B}_{w}$ is $B w U_{m}$, and by (B.9) we have

$$
\operatorname{supp}_{B w U_{m}} W_{m}=\left(\operatorname{supp}_{B w} W_{m}\right) U_{m} .
$$

Therefore, Lemma B.11. implies that

$$
\inf \left|\alpha_{0}\right|\left(\operatorname{supp}_{B w U_{m}} W_{m}\right)>0
$$

for all $\alpha \in S^{\circ}(w)$ as well.

It remains to prove (B.11). By (B.9), we may replace $\mathcal{B}_{w}(i, m)$ in (B.11) by the set

$$
\mathcal{B}_{w}^{\prime}(i, m)=\left\{b \bar{w} n: b \in B, n \in N_{w}^{-}, n_{i}=1, n_{i+1} \notin U_{m}\right\} .
$$

Let $M$ (depending on $K$ ) such that (B.11) holds for all $j<i$ and (B.10) holds for all $w^{\prime}<w$. We choose $M_{1} \geq M$ depending only on $K$ such that

$$
n_{-\beta_{i}}\left(\beta_{i}\left(a^{-M_{1}}\right) \mathcal{O}\right) \subset \bigcap_{n \in U_{M}} n K n^{-1}
$$

when $\alpha_{i}$ if of type (I), and

$$
\left\{n_{-\beta_{i}}\left(\tilde{\beta}_{i}\left(a^{-M_{1}}\right) p, \tilde{\beta}_{i}\left(a^{-2 M_{1}}\right) q\right): p, q \in \mathcal{O}_{E_{\alpha_{i}}}, N_{E_{\alpha_{i}} / E_{\alpha_{i}}}(p)=-\operatorname{Tr}_{E_{\alpha_{i}} / F_{\alpha_{i}}}(q)\right\} \subset \bigcap_{n \in U_{M}} n K n^{-1}
$$

when $\alpha_{i}$ if of type (II) for $\beta_{i}=w_{i}^{-1} \alpha_{i}$.

Assume that $W \in \Omega\left(N \backslash G, \psi_{N}\right)^{K}, g \in \mathcal{B}_{w}^{\prime}(i, m)$, and $W_{m}(g) \neq 0$ with $m \geq M_{1}$. Write $g=t \bar{w} n$. Since

$$
W_{m}(g)=\frac{1}{\operatorname{vol}\left(U_{m}\right)} \int_{U_{m}} W_{M}\left(g n^{\prime}\right) \psi_{N}\left(n^{\prime}\right)^{-1} d n^{\prime}=
$$

there exists $n^{\prime} \in U_{m}$ such that $W_{M}\left(g n^{\prime}\right) \neq 0$. Let $n n^{\prime}=n_{+} \tilde{n}$ where $n_{+} \in N_{w}^{+}$and $\tilde{n} \in N_{w}^{-}$. Write

$$
\tilde{n}= \begin{cases}{ }_{i} \tilde{n} n_{\beta_{i}}\left(\tilde{x}_{i}\right) \tilde{n}_{i} & \text { if } \alpha_{i} \text { is of type (I), } \\ { }_{i} \tilde{n} n_{\beta_{i}}\left(\tilde{r}_{i}, \tilde{m}_{i}\right) \tilde{n}_{i} & \text { if } \alpha_{i} \text { is of type (II). }\end{cases}
$$

From our assumption on $n$ and $n^{\prime}$, by Corollary B.1 B.2 we have $\left|\tilde{x}_{i}\right|>\left|\beta_{i}(a)^{m}\right|$ and $\left|\tilde{m}_{i}\right|>\left|\beta_{i}(a)^{2 m}\right|$. In particular, $\tilde{n}_{i+1} \notin U_{m}$ by $(\underline{B .6})$.

Let $j \leq i+1$ be the smallest index for which $\tilde{n}_{j} \notin U_{M}$. If $j \leq i$, then $\tilde{g} \in \mathcal{B}_{w}(j-1, M)$. Thus, we may apply our inductive assumption, and by the choice of $M$ we have $\left|\alpha_{0}(\tilde{g})\right| \geq \delta_{1}$ for all $\alpha \in S^{\circ}(w)$ where $\delta_{1}>0$ depends only on $W$. Hence, by (B.1) we also have $\left|\alpha_{0}(g)\right| \geq \delta_{2}$ for a suitable constant $\delta_{2}=\delta_{2}(m, W)>0$.

Assume that $j=i+1$. Then $\tilde{n}_{i} \in U_{M}$ and therefore $W_{M}\left(g^{\prime}\right) \neq 0$ where

$$
g^{\prime}= \begin{cases}t \bar{w}_{i} \tilde{n} n_{\beta_{i}}\left(\tilde{x}_{i}\right) & \text { if } \alpha_{i} \text { is of type (I), } \\ t \bar{w}_{i} \tilde{n} n_{\beta_{i}}\left(\tilde{r}_{i}, \tilde{m}_{i}\right) & \text { if } \alpha_{i} \text { is of type (II). }\end{cases}
$$


Here, we note that $g^{\prime} \in N \tilde{g} \tilde{n}_{i}^{-1}$ since $t w\left(n_{+}\right)(t w)^{-1}$. On the other hand, since $\tilde{n}_{i+1} \notin U_{M}$, we get

$$
\begin{cases}x_{i}^{-1} \in \beta_{i}\left(a^{-m}\right) \mathcal{O}_{F_{\alpha_{i}}} & \text { if } \alpha_{i} \text { is of type (I), } \\ m_{i}^{-1} \in \beta_{i}\left(a^{-m}\right) \mathcal{O}_{E_{\alpha_{i}}} & \text { if } \alpha_{i} \text { is of type (II), }\end{cases}
$$

and thus $W_{M}$ is right invariant by $n_{-\beta_{i}}\left(-\tilde{x}_{i}^{-1}\right)$ (resp. $\left.n_{-\beta_{i}}\left(-r m^{-1}, \bar{m}^{-1}\right)\right)$ if $\alpha_{i}$ is of type (I) (resp. type (II)) since these belong to $\cap n \in U_{M} n K n^{-1}$ by the choice of $M_{1}$. Hence, $W_{M}\left(g^{\prime \prime}\right) \neq 0$ where $g^{\prime \prime}:=g^{\prime} n_{-\beta_{i}}\left(-\tilde{x}_{i}^{-1}\right)\left(\right.$ resp. $g^{\prime} n_{-\beta_{i}}\left(-r m^{-1}, \bar{m}^{-1}\right)$ ) if $\alpha_{i}$ is of type (I) (resp. type (II)). By the first part of Lemma B.5, B.6, we have $g^{\prime \prime} \in B w^{\prime} B$ with $w^{\prime}<w$. We conclude from the choice of $M$ that $\left|\alpha_{0}\right|\left(g^{\prime}\right)=\left|\alpha_{0}\right|\left(g^{\prime \prime}\right) \geq \delta_{3}$ for all $\alpha \in S^{\circ}\left(w^{\prime}\right)$ for a suitable constant $\delta_{3}=\delta_{3}(W, M)$ by our inductive assumption. In particular, this holds for all $\alpha^{\circ}(w)$. Once again by (B.1) we infer that $\left|\alpha_{0}\right|(g) \geq \delta_{4}$ for a suitable constant $\delta_{4}=\delta_{4}(W, m)$. This concludes the proof of Proposition B.2

B.6. Non-vanishing of Bessel functions. Suppose that $\mathbf{G}$ is quasi-split reductive group over $F$. We shall use the same notation as above. Put $B_{0}=A \ltimes N$ and $G^{\circ}=B_{0} w_{0} B_{0}$.

Theorem B.3. For any tempered $\pi \in \operatorname{Irr}_{\mathrm{gen}, \psi_{N}} G$, the function $\mathbb{B}_{\pi}$ is not identically zero on $G^{\circ}$.

Let $A^{d}=A \cap G^{\text {der }}$. Consider the family

$$
A_{n}=\left\{t \in A^{d}:|\alpha(t)-1| \leq q^{-n} \text { for all } \alpha \in \triangle_{0}\right\} \in \mathcal{C} \mathcal{S G R}\left(A^{d}\right)
$$

which forms a basis of neighborhoods of 1 of $A^{d}$.

Let $U_{n}$ be the group generated by $\left\langle n_{\alpha}\left(\varpi^{-n} \mathcal{O}_{F_{\alpha}}\right), n_{\beta}\left(\varpi^{-n} \mathcal{O}_{E_{\beta}}, \varpi^{-2 n} \mathcal{O}_{E_{\beta}}\right): \alpha \in \triangle_{0}^{(I)}, \beta \in \triangle_{0}^{(I I)}\right\rangle$.

Lemma B.12. For any $u \in N$ we have

$$
\left(\operatorname{vol}\left(A_{n}\right)\right)^{-1} \int_{A_{n}} \psi_{N}\left(t u t^{-1}\right) d t= \begin{cases}\psi_{N}(u) & u \in N_{n} \\ 0 & \text { otherwise }\end{cases}
$$

In the same argument as the proof of [30, Theorem A.1], we can prove Theorem B.3 substituting $U_{n}$ for $N_{n}$ in the argument [30, Appendix A].

\section{REFERENCES}

[1] U. K. Anandavardhanan, Root numbers of Asai L-functions. Int. Math. Res. Not. IMRN 2008, Art. ID rnn125, 25 pp.

[2] U. K. Anandavardhanan, A. C. Kable, Anthony and R. Tandon, Distinguished representations and poles of twisted tensor L-functions. Proc. Amer. Math. Soc. 132 (2004), no. 10, 2875-2883.

[3] J. Arthur, The endoscopic classication of representations. Orthogonal and symplectic groups. Amer. Math. Soc. Colloq. Publ. 61, xviii+590 pp. Amer. Math. Soc., Providence, R.I. (2013)

[4] H. Atobe, On the Uniqueness of Generic Representations in an L-Packet, to appear in IMRN, doi: 10.1093/imrn/rnw220

[5] E. M. Baruch, Bessel Functions for GL(n) Over a p-Adic Field. Automorphic Representations, L-functions and Applications: Progress and Prospects, 1-40. Ohio State University Mathematical Research Institute Publications 11. Berlin: de Gruyter, 2005.

[6] A. Ben-Artzi and D. Soudry, On L-functions for $\mathrm{U}_{2 k} \times \mathrm{R}_{E / F} \mathrm{GL}_{m},(k<m)$. Advances in the theory of automorphic forms and their L-functions, 69-104, Contemp. Math., 664, Amer. Math. Soc., Providence, RI, 2016.

[7] R. Beuzart-Plessis, Plancherel formula for $\mathrm{GL}_{n}(F) \backslash \mathrm{GL}_{n}(E)$ and applications to the Ichino-Ikeda and formal degree conjectures for unitary groups. arXiv: 1812.00047

[8] I.N. Bernstein and A.V. Zelevinsky, Induced representations of reductive p-adic groups. I. Ann. Sci. École Norm. Sup., (4) 10, no. 4, 441-472 (1977)

[9] P-H. Chaudouard and G. Laumon, Le lemme fondamental pondéré. II. Énoncés cohomologiques. Ann. of Math. (2) 176 (2012), no. 3 , $1647-1781$.

[10] P. Delorme, Formule de Plancherel pour les fonctions de Whittaker sur un groupe réductif p-adique. Ann. Inst. Fourier (Grenoble) 63 (2013), no. 1, 155-217.

[11] M. Furusawa and K. Morimoto, Refined global Gross-Prasad conjecture on special Bessel periods and Boecherer's conjecture. to appear in J. Eur. Math. Soc. (JEMS).

[12] W. T. Gan and A. Ichino, Formal degrees and local theta correspondence. Invent. Math. 195 (2014), no. 3, 509-672.

[13] S. Gelbart and J. D. Rogawski, L-functions and Fourier-Jacobi coefficients for unitary group U(3). Invent. Math. 105 (1991), no. 3, 445-472.

[14] D. Ginzburg, S. Rallis, and D. Soudry, The descent map from automorphic representations of GL( $n$ ) to classical groups. World Scientific Publishing Co. Pte. Ltd., Hackensack, NJ, 2011. x+339 pp.

[15] B. Gross and D. Prasad, On the decomposition of a representation of $\mathrm{SO}_{n}$ when restricted to $\mathrm{SO}_{n-1}$. Canad. J. Math. 44, 974-1002 (1992)

[16] B. Gross and M. Reeder, Arithmetic invariants of discrete Langlands parameters. Duke Math. J. 154 (2010), no. 3, $431-508$.

[17] G. Henniart, Caractérisation de la correspondance de Langlands locale par les facteurs $\varepsilon$ de paires. Invent. Math. 113 (1993), 339-350.

[18] K. Hiraga, A. Ichino and T. Ikeda, Formal degrees and adjoint $\gamma$-factors. J. Amer. Math. Soc. 21 (2008), no. 1, $283-304$.

[19] A. Ichino, and T. Ikeda, On the periods of automorphic forms on special orthogonal groups and the Gross-Prasad conjecture. Geom. Funct. Anal. 19 (2010), no. 5, 1378-1425.

[20] A. Ichino, E. Lapid, and Z. Mao, On the formal degrees of square-integrable representations of odd special orthogonal and metaplectic groups. Duke Math. J. 166 (2017), no. 7, 1301-1348.

[21] H. Jacquet, I. I. Piatetski-Shapiro, and J. Shalika, Rankin-Selberg convolutions. Amer. J. Math. 105 (1983), 367-464. 
[22] D. Jiang and D. Soudry, Generic representation and local Langlands reciprocity law for p-adic $\mathrm{SO}_{2 n+1}$. Contributions to automorphic forms, geometry, and number theory, 457-519, Johns Hopkins Univ. Press, Baltimore, MD, 2004.

[23] A. C. Kable, Asai L-functions and Jacquet's conjecture. Amer. J. Math. 126 (2004), no. 4, 789-820.

[24] T. Kaletha, A. Minguez, S.W., Shin ad P.-J., White, Endoscopic classification of representations: inner forms of unitary groups. arXiv:1409.3731

[25] H. Kim and M. Krishnamurthy, Stable base change lift from unitary groups to GL ${ }_{n}$. IMRP Int. Math. Res. Pap. 2005, no. 1, 1-52.

[26] M. Kneser Semi-simple algebraic groups. Algebraic Number Theory (Proc. Instructional Conf., Brighton, 1965), 250-265, Thompson, Washington, D.C., 1967.

[27] T. Konno, A note on Langlands classification and irreducibility of induced representations of p-adic group Kyushu J. Math. 57 (2003), no. 2, 383-409.

[28] E. Lapid and Z. Mao, Stability of certain oscillatory integrals, Int. Math. Res. Not. IMRN (2013), no.3, 525-547.

[29] E. Lapid and Z. Mao, On Whittaker-Fourier coefficients of automorphic forms on unitary groups : reduction to a local conjecture. Advances in the theory of automorphic forms and their L-functions, 295-320, Contemp. Math., 664, Amer. Math. Soc., Providence, RI, 2016.

[30] E. Lapid and Z. Mao, On an analogue of the Ichino-Ikeda conjecture for Whittaker coefficients on the metaplectic group. Algebra Number Theory 11 (2017), no. 3, 713-765.

[31] E. Lapid and Z. Mao, Whittaker-Fourier coefficients of cusp forms on $\widetilde{\mathrm{Sp}}_{n}$ : reduction to a local statement, Amer. J. Math. 139 (2017), no. $1,1-55$.

[32] E. Lapid and Z. Mao, On a new functional equation for local integrals. Automorphic forms and related geometry: assessing the legacy of I. I. Piatetski-Shapiro, 261-294, Contemp. Math., 614, Amer. Math. Soc., Providence, RI, 2014.

[33] E. Lapid and Z. Mao, A conjecture on Whittaker-Fourier coefficients of cusp forms J. Number Theory 146 (2015), $448-505$.

[34] E. Lapid and Z. Mao, Model transition for representations of metaplectic type. With an appendix by Marko Tadić. Int. Math. Res. Not. IMRN 2015, no. 19, 9486-9568.

[35] B. Liu, Genericity of representations of $p$-adic $\mathrm{Sp}_{2 n}$ and local Langlands parameters., Canad. J. Math. 63 (2011), no. 5, $1107-1136$.

[36] N. Matringe, Conjectures about distinction and local Asai L-functions. Int. Math. Res. Not. IMRN 2009, no. 9, $1699-1741$.

[37] N. Matringe, Distinguished generic representations of GL(n) over p-adic fields. Int. Math. Res. Not. IMRN 2011, no. 1, 74-95.

[38] C. Moeglin, and M. Tadić. Construction of discrete series for classical p-adic groups. J. Amer. Math. Soc. 15 (2002), no. 3, $715-786$.

[39] C. Moeglin, M. F. Vigneras and J.-L., Waldspurger Correspondances de Howe sur un corps p-adique. Lecture Notes in Mathematics, 1291. Springer-Verlag, Berlin, 1987. viii+163 pp.

[40] C. Moeglin and J.-L., Waldspurger, Stabilisation de la Formule des Traces Tordue. Volume 1., Progr. Math. 316, Birkhäuser/Springer, Cham, 2016.

[41] C. Moeglin and J.-L., Waldspurger, Stabilisation de la Formule des Traces Tordue. Volume 2., Progr. Math. 317, Birkhäuser/Springer, Cham, 2016.

[42] C.P. Mok, Endoscopic classification of representations of quasi-split unitary groups. Mem. Am. Math. Soc. 235, 1108 (2015)

[43] K. Morimoto, On the irreducibility of global descents for even unitary groups and its applications. Trans. Amer. Math. Soc. 370 (2018), no. 9, 6245-6295.

[44] K. Morimoto, Model transition for representations of unitary type. to appear in IMRN, https://doi.org/10.1093/imrn/rny048

[45] K. Morimoto, On gamma factors of Rankin-Selberg integrals for $\mathrm{U}_{2 \ell} \times \mathrm{GL}_{n}$. Preprint

[46] O. Offen, On local root numbers and distinction J. Reine Angew. Math. 652 (2011), 165-205.

[47] Y. Sakellaridis and A. Venkatesh, Periods and harmonic analysis on spherical varieties. Astérisque No. 396 (2017), viii+360 pp.

[48] F. Shahidi, On multiplicativity of local factors. Festschrift in honor of I. I. Piatetski-Shapiro on the occasion of his sixtieth birthday, Part II (Ramat Aviv, 1989), 279-289, Israel Math. Conf. Proc., 3, Weizmann, Jerusalem, 1990.

[49] F. Shahidi, A proof of Langlands conjecture on Plancherel measures; complementary series for p-adic groups. Ann. of Math. 132 (1990), 273-330.

[50] D. Soudry and Y. Tanay, On local descent for unitary groups. J. Number Theory 146 (2015), 557-626.

[51] R. Steinberg, Lectures on Chevalley Groups. Notes prepared by John Faulkner and Robert Wilson. Yale University, New Haven, Conn., 1968. iii $+277 \mathrm{pp}$.

[52] N. R. Wallach, Real Reductive Groups, I, Pure Appl. Math. 132, Academic Press, Boston,1988.

[53] N. R. Wallach, Real Reductive Groups, II, Pure Appl. Math. 132, Academic Press, Boston,1992.

[54] A. V. Zelevinsky, Induced representations of reductive p-adic groups. II. On irreducible representations of GL( $n)$ Ann. Sci. École Norm. Sup. (4) 13 (1980), no. 2, 165-210. 